\title{
Experimental and Computational Exploration of the Dilute Magnetic Delafossite CuAl1-xFexO2 Alloys
}

\author{
Mina Aziziha \\ West Virginia University, miaziziha@mix.wvu.edu
}

Follow this and additional works at: https://researchrepository.wvu.edu/etd

Part of the Condensed Matter Physics Commons

\section{Recommended Citation}

Aziziha, Mina, "Experimental and Computational Exploration of the Dilute Magnetic Delafossite CuAl1-xFexO2 Alloys" (2020). Graduate Theses, Dissertations, and Problem Reports. 7931.

https://researchrepository.wvu.edu/etd/7931

This Dissertation is protected by copyright and/or related rights. It has been brought to you by the The Research Repository @ WVU with permission from the rights-holder(s). You are free to use this Dissertation in any way that is permitted by the copyright and related rights legislation that applies to your use. For other uses you must obtain permission from the rights-holder(s) directly, unless additional rights are indicated by a Creative Commons license in the record and/ or on the work itself. This Dissertation has been accepted for inclusion in WVU Graduate Theses, Dissertations, and Problem Reports collection by an authorized administrator of The Research Repository @ WVU.

For more information, please contact researchrepository@mail.wvu.edu. 


\title{
Experimental and Computational Exploration of the Dilute
}

\section{Magnetic Delafossite $\mathrm{CuAl}_{1-x} \mathrm{Fe}_{x} \mathrm{O}_{2}$ Alloys}

\section{Mina Aziziha}

Dissertation submitted to the Eberly

College of Arts and Sciences at West

Virginia University in partial fulfillment

of the requirements for the degree of

\section{Doctor of Philosophy \\ in}

Physics

\author{
Matthew B. Johnson, Ph.D., Chair \\ Alan D. Bristow, Ph.D. \\ Mikel B. Holcomb, Ph.D. \\ Edward M. Sabolsky, Ph.D. \\ Department of Physics and Astronomy \\ Morgantown, West Virginia \\ 2020
}

Keywords: Complex Oxides, Delafossite, Dilute Alloys, Magnetism, Optical, Phonon, Crystallography, Elemental and Chemical Characterization, Density Functional Theory, Electronic Band Structure, Phonon Dispersion, Pramagnetism

Copyright 2020, Mina Aziziha 


\section{Abstract \\ Experimental and Computational Exploration of the Dilute Magnetic Delafossite $\mathrm{CuAl}_{1-x} \mathrm{Fe}_{x} \mathrm{O}_{2}$ Alloys}

Mina Aziziha

$\mathrm{CuAlO}_{2}$ is among several ternary delafossites, which is a rare $p$-type semiconductor with potential applications as a transparent conductive oxide, photocatalyst, and spintronics when doped with transition metal ions. Reported in this thesis are results from our investigations of $\mathrm{CuAl}_{1-x} \mathrm{Fe}_{x} \mathrm{O}_{2}(x=0$ to1) with a focus on the $x$-dependence of structural, magnetic, vibrational, optical properties, and the role of defects and impurities. Samples are prepared by solid-state reactions.

We performed a complete study of magnetic properties to investigate the possibility of room temperature ferromagnetic alloys, which are used in transparent ferromagnet applications, suggested by a computational study. Analysis of magnetization $(M) v s$. temperature ( $T$, from 2 to $300 \mathrm{~K}$ ) data by CurieWeiss law confirms $\mathrm{Fe}^{3+}$ as the electronic state of $\mathrm{Fe}$; this analysis also yields a negative $\theta$ characteristic of an antiferromagnetic $\mathrm{Fe}^{3+}-\mathrm{Fe}^{3+}$ exchange coupling and magnitudes of $x$ in good agreement with the nominal values. The isothermal $M v s$. $H$ (up to $H=90 \mathrm{kOe}$ ) data analyzed by the modified Brillouin function support the results obtained from the $M v s . T$ analysis. High-resolution $M-H$ loop measurements at $300 \mathrm{~K}$ and $10 \mathrm{~K}$ show negligible coercivity $\left(H_{\mathrm{C}}\right)$ at $10 \mathrm{~K}$ but $H_{\mathrm{C}} \sim 100 \mathrm{Oe}$ at $300 \mathrm{~K}$. The results suggest that the room temperature ferromagnetism can originate from hematite impurity, but not for $\mathrm{CuAl}_{1-x} \mathrm{Fe}_{x} \mathrm{O}_{2}$ alloys.

The understanding of the phonon dynamics of alloys is crucial because they have a fundamental indirect bandgap. I introduce a new approach, which is applicable to anisotropic, dilute alloys with allowance for a large variety of alloying elements. This approach has significant advantages over previously reported methods, especially for the lattice dynamics of such complex alloys. We use this approach to model the effects of $\mathrm{Fe}$-doping on the vibrational modes in dilute alloys of $\mathrm{CuAl}_{1-x} \mathrm{Fe}_{x} \mathrm{O}_{2}(x=0-0.10)$ delafossite powders. Raman and FTIR spectroscopies are performed to measure optical phonon frequencies. For the phonon calculations, an approach using a disordered supercell is not feasible because it is computationally expensive. Instead, we developed our weighted dynamical matrix (WDM) approach that uses a straightforward ordered supercell for force-constant calculations of the $\mathrm{CuAlO}_{2}$ and $\mathrm{CuFeO}_{2}$ parent endpoints and combines them using a WDM approach. Computationally, when Fe is substituted for Al (increasing $x$ ), an increase in the bond length is observed, leading to a redshift in the peak positions in all the phonon modes $v s . x$, in agreement with the experimentally observed trend.

$\mathrm{CuAlO}_{2}$, with an indirect bandgap $\sim 3 \mathrm{eV}$, is a good candidate for photocatalysis applications because of its chemical stability and absorption in the solar region. However, efforts to improve its optical absorption continue. Here, I report the effect of alloying on the optical absorption of $\mathrm{CuAl}_{1-x} \mathrm{Fe}_{x} \mathrm{O}_{2}(x=0.0$ $1.0)$ by measuring the optical absorption of alloys from 1 to $6 \mathrm{eV}$ and comparing these results to electronic band structures calculated using density functional theory (DFT) calculations. The calculations use DFT $+U$ supercell methods, including spin. Experimentally, we observe a new absorption feature associated with $\mathrm{Fe}$ at about $1.8 \mathrm{eV}$ for $x=0.01$ shifting to $1.4 \mathrm{eV}$ for $x=0.10$. The energy of this feature and its redshift with $x$ agrees with calculated spin-down Fe-3d states. This added feature will lead to more optical absorption.

The major conclusions from this research are that by alloying $\mathrm{CuAlO}_{2}$ with $\mathrm{Fe}$, the optical absorption will improve. Also, we proposed a straightforward, computationally efficient WDM approach, which confirms the redshift associated with the optical phonon modes. With our magnetic measurements, we confirmed that the dilute alloys are not ferromagnetic at room temperature. 


\section{Acknowledgment}

My Ph.D. journey was an excellent experience that prepared me for my future career. I would like to thank the people who helped me complete the program and the research presented here.

First and foremost, I am so grateful to my advisor and committee chair, Dr. Matthew B. Johnson, for his priceless guidance, support, and encouragement. He has been the best mentor, and I have learned infinitely from him. His open mind helped us to progress in the research and make various collaborations. I am so thankful to Dr. Mohindar S. Seehra for his support and guidance. He helped me publish numerous papers and to build up collaborations. I would like to thank my committee members Dr. Mikel B. Holcomb, Dr. Alan D. Bristow, and Dr. Edward Sabolsky, for their time and efforts put towards this work.

Additionally, I would like to thank Harley Hart, Dr. Qiang Wang, and Dr. Marcela Redigolo from Shared Research Facilities. They maintained the instruments used in this work and trained me on them. I would also like to thank our collaborators Dr. Mikel B. Holcomb, Dr. Alan D. Bristow, and Dr. Edward Sabolsky, Dr. Aldo H. Romero, Dr. James Lewis, and Dr. Lian-Shin Lin at West Virginia University, as

well as Dr. Subhash Thota from Indian Institute of Technology, and Dr. Suresh Pittala from Indian Institute of Science who helped me to have a more prolific Ph.D.

I owe my thanks to my parents and sisters, whose support has motivated me to follow my dreams. I am so grateful to my devoted husband, Saeed Akbarshahi, who has been my rock and helped me through the research by performing the calculations. 


\section{Table of Contents}

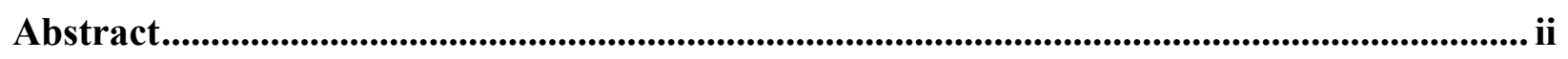

Experimental and Computational Exploration of the Dilute Magnetic Delafossite $\mathrm{CuAl}_{1}$ -

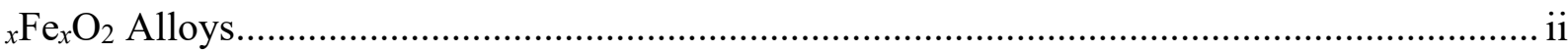

Acknowledgment.......................................................................................................................................... iii

Table of Contents ..................................................................................................................................... iv

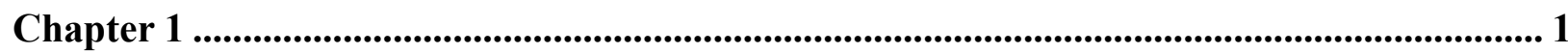

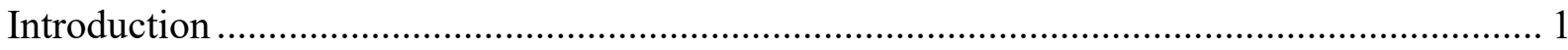

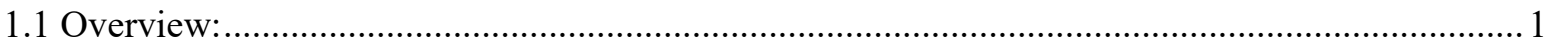

1.2 Motivation and Possible Application ................................................................................

1.3 Overview of Modeling for Dilute Alloys:............................................................................. 4

1.4 Goal of the Thesis: …………………………………………………………………....

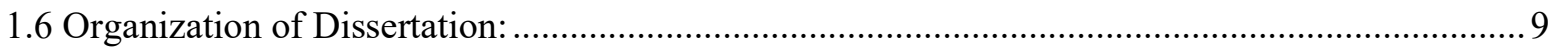

Chapter 2 ................................................................................................................................. 10

Preparation of Samples and their Structural Characterization ............................................... 10

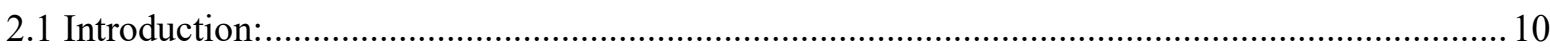

2.2 Overview of Procedures for Synthesis and Growth of Delafossites ...........................................11

2.2.1 Synthesis of Polycrystalline Powders: ............................................................................... 11

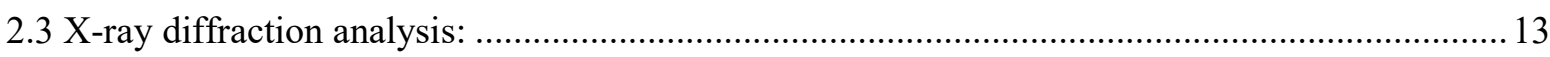

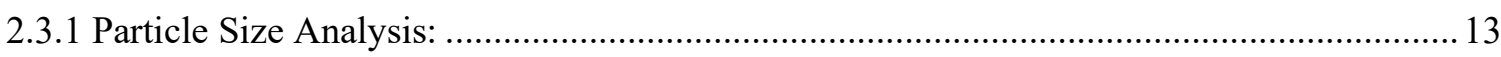

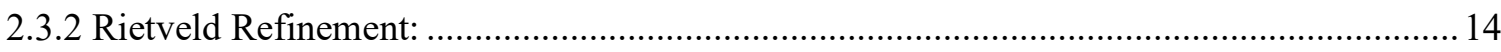

2.3.3 Variation of Lattice Parameters by increasing Fe concentration: ........................................ 17

2.4 Elemental Analysis by Scanning Electron Microscopy (SEM): .................................................. 19

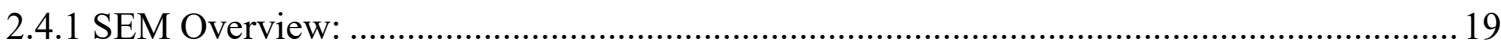

2.5 Delafossite Alloys Topography and Elemental Characterization: …………………………......2

2.6 Chemical Analysis by X-ray Photoelectron Spectroscopy (XPS): ...............................................25

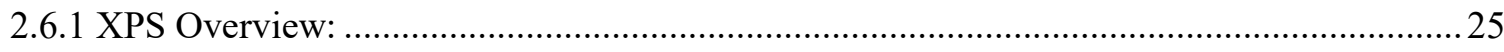

2.6.2 Delafossite Alloys Chemical Analysis:.............................................................................2 27

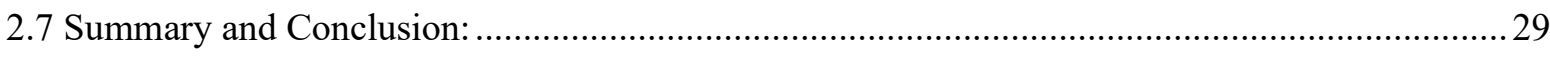

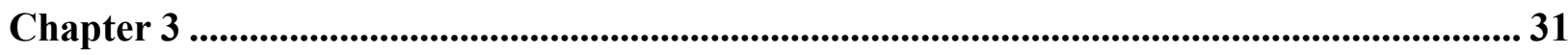

Computational Study of Dilute Delafossite alloys ..................................................................... 31 


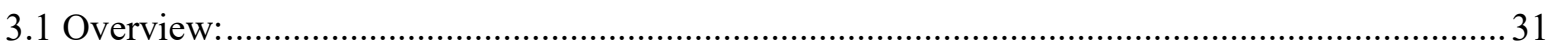

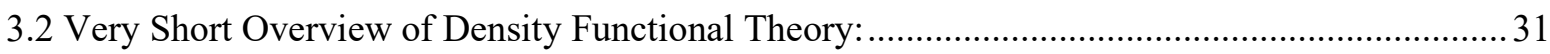

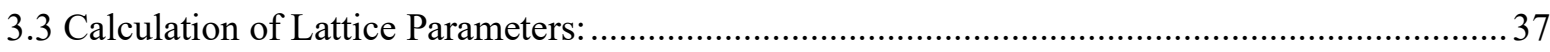

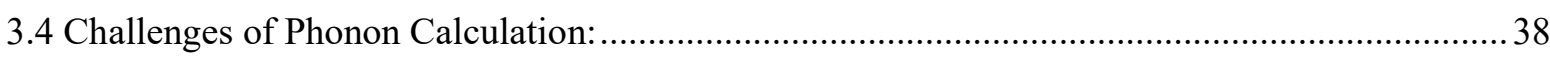

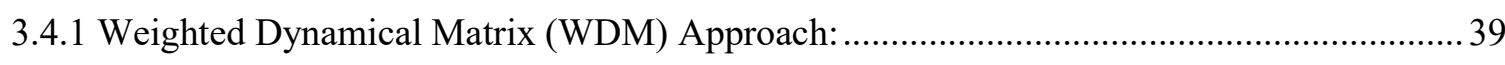

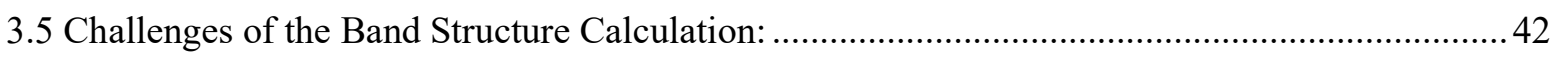

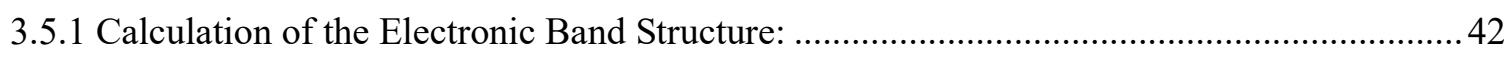

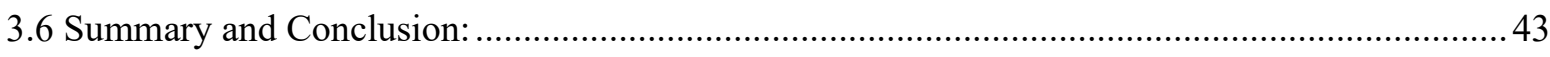

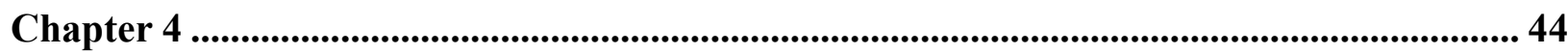

Variation of Magnetic Properties of Delafossite Alloys: ................................................. 44

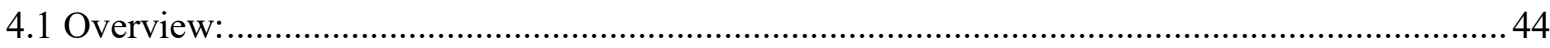

4.2 Introduction:

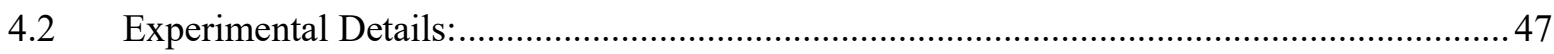

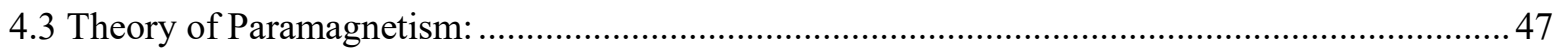

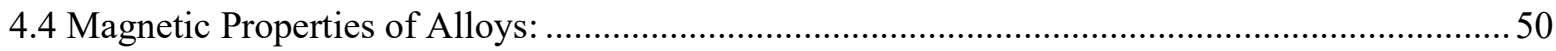

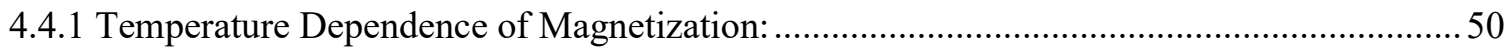

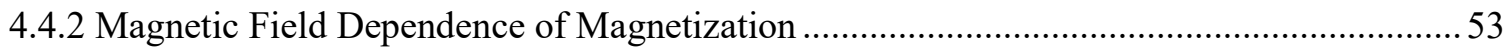

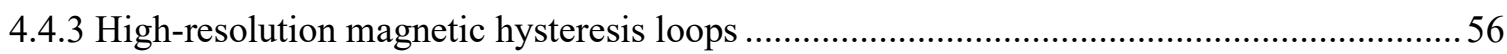

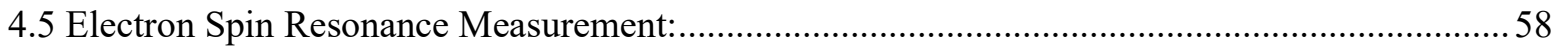

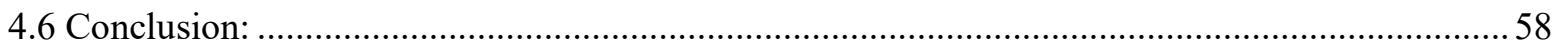

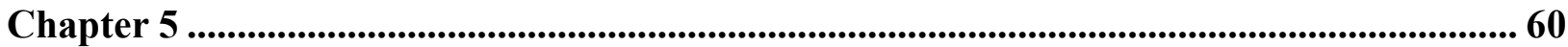

Phonon Dynamics in Dilute $\mathrm{CuAl}_{1-x} \mathrm{Fe}_{x} \mathrm{O}_{2}$ Delafossite Alloys by a Weighted Dynamical

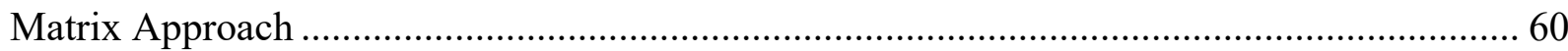

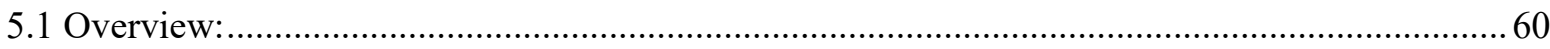

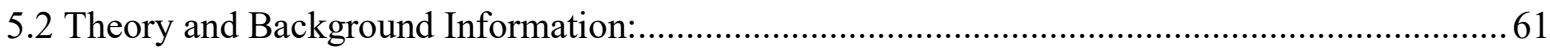

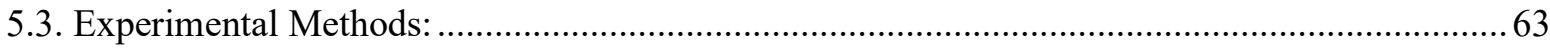

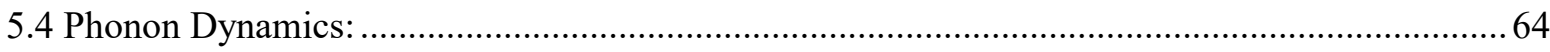

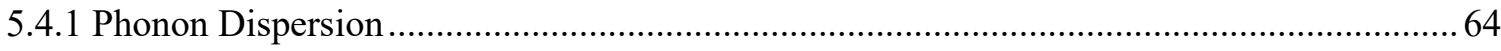

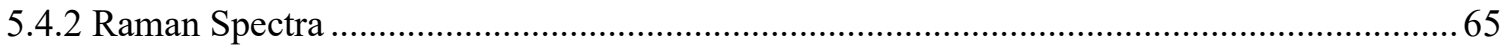

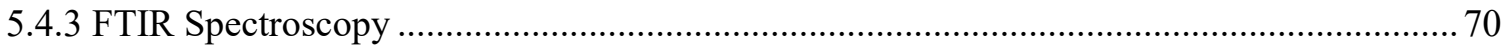

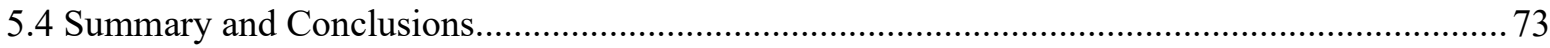

Chapter 6 ................................................................................................................................... 75

Optical Properties of Delafossite Alloys ….................................................................. 75 
6.1 Overview:

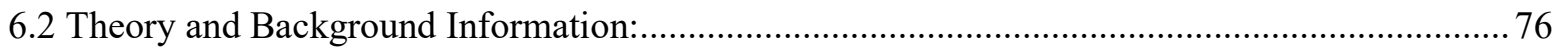

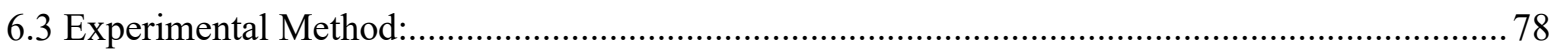

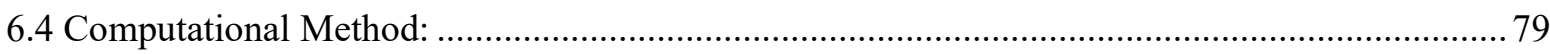

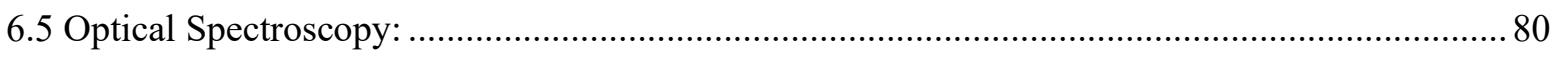

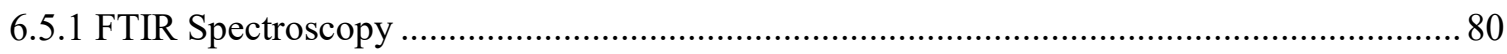

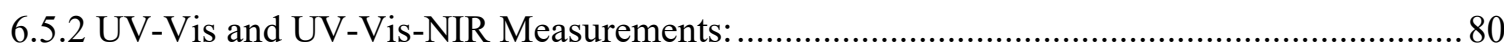

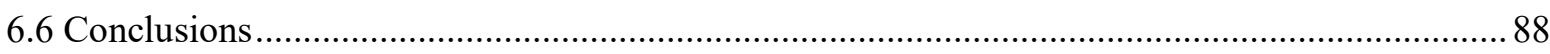

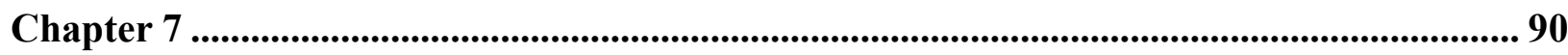

Conclusion, Future Direction, and Publications................................................................. 90

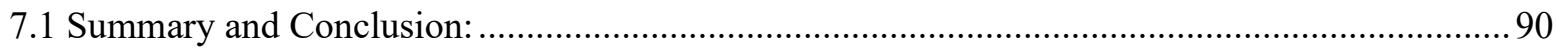

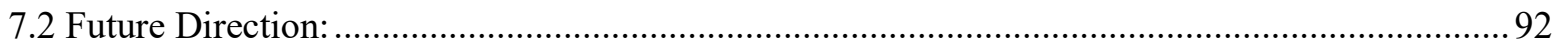

7.3 Publications of the Author Related to this Dissertation: ........................................................... 93

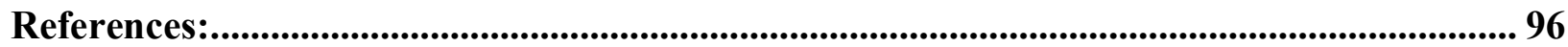




\section{Chapter 1}

\section{Introduction}

\subsection{Overview:}

In this chapter, the properties and applications of $\mathrm{CuAlO}_{2}$ are described. The first principle calculations contribution to understanding the optical and electronic and the vibrational properties of the samples are explained. The possible applications for the Fe-alloyed $\mathrm{CuAlO}_{2}$ are described.

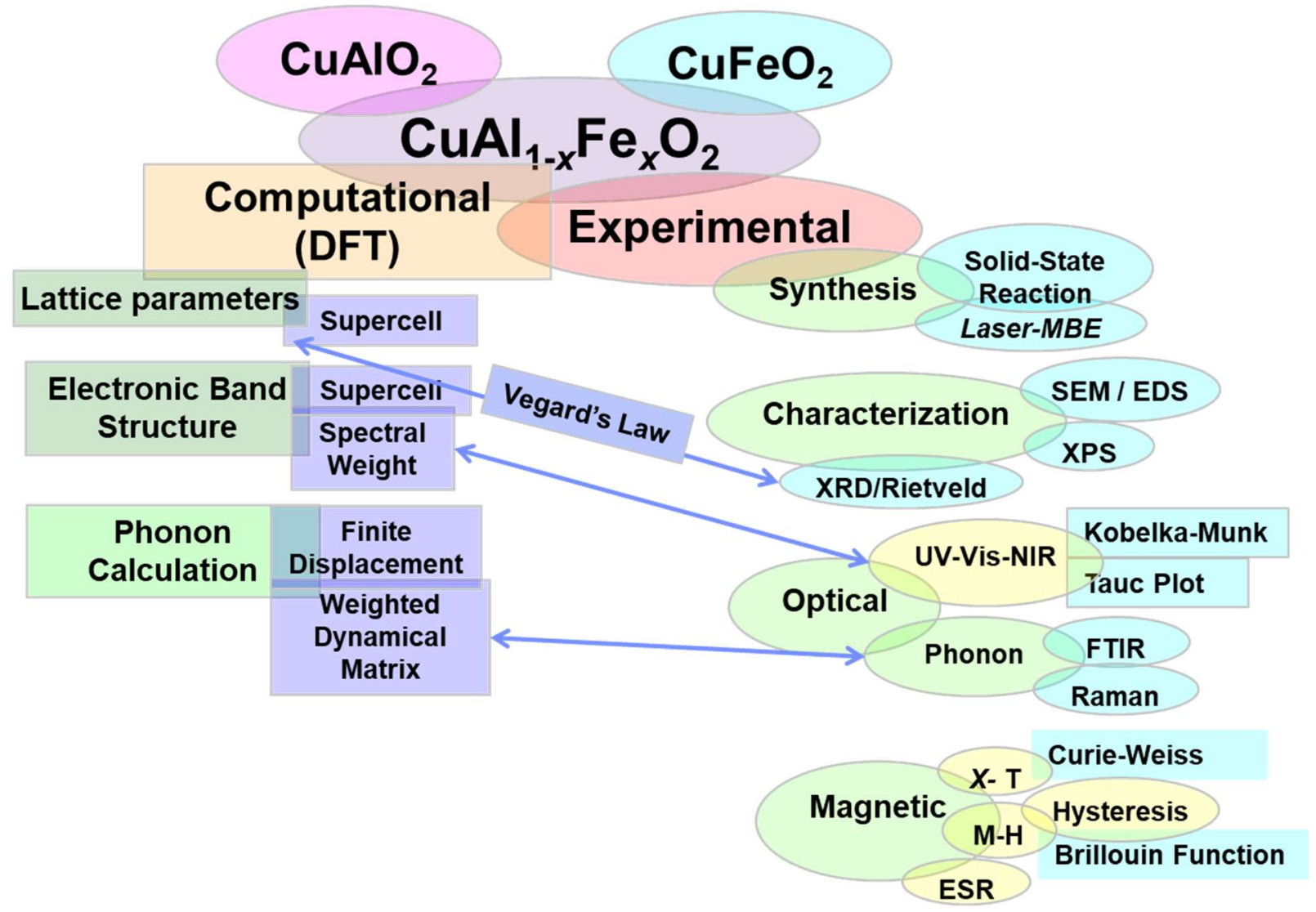

Figure 1.1: The bubble graph shows the scheme of the thesis based on computational and experimental data on $\mathrm{CuAlO}_{2}, \mathrm{CuAl}_{1-x} \mathrm{Fe}_{x} \mathrm{O}_{2}$, and $\mathrm{CuFeO}_{2}$.

\subsection{Motivation and Possible Application}

Oxide semiconductors are a very promising family of materials for electronic device applications because of their large optical bandgap, such as energy-conversion devices, photocatalysis, and display unique physical properties. They can be found in both n-type and p-type making them attractive candidates 


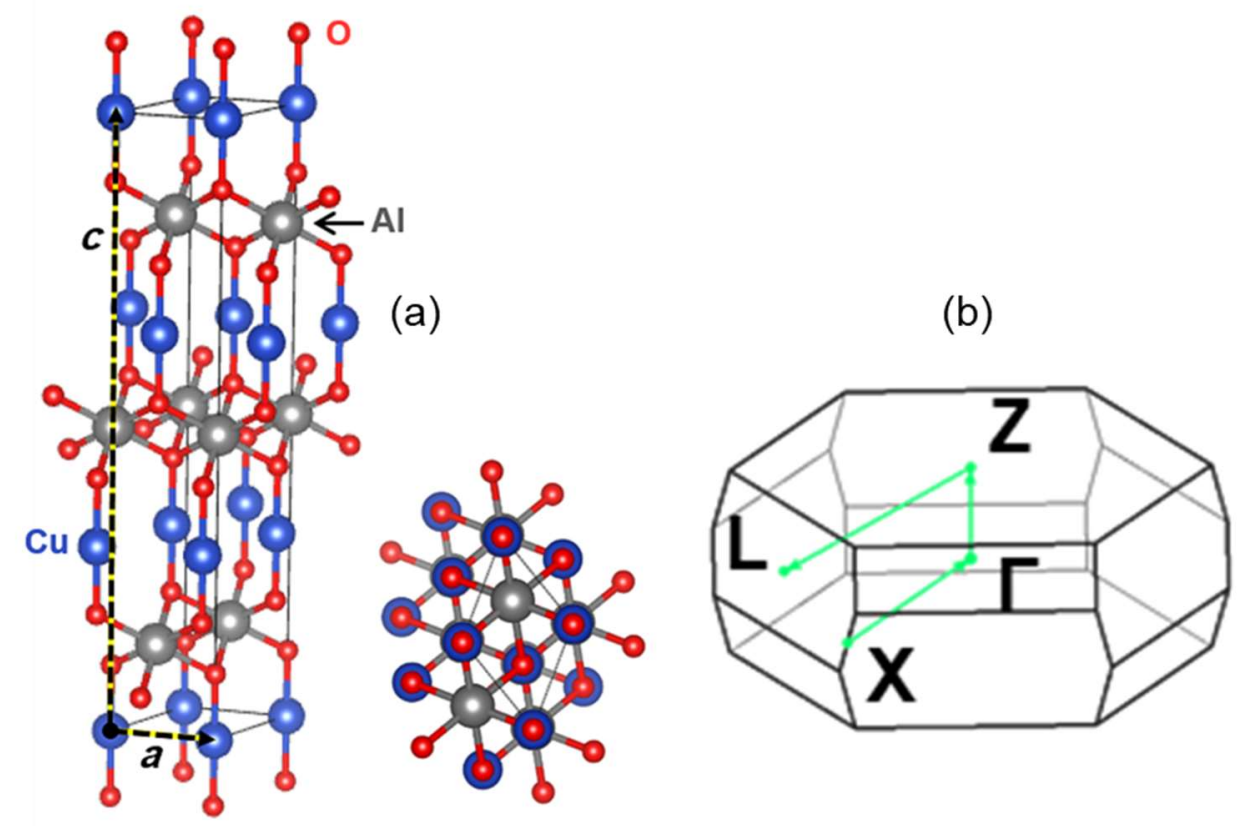

Figure 1.2: (a) Rhombohedral crystal structure of $\mathrm{CuAlO}_{2}$ and(b) the first Brillouin zone of the $\mathrm{CuAlO} 2$ based on the primitive unit cell.

for transparent conducting oxide, photodetectors, and transparent thin-film transistors. Other properties, such as large exciton binding energies and the availability of a large selection of substrates, makes it superior over other materials such as nitride semiconductors. The main disadvantage of many oxide semiconductors is that their electrical conductivity is not easily controllable, unlike traditional semiconductors such as $\mathrm{Si}, \mathrm{Ge}$, or GaAs.Oxides such as $\mathrm{ZnO}, \mathrm{Ga}_{2} \mathrm{O}_{3}, \mathrm{In}_{2} \mathrm{O}_{3}, \mathrm{SnO}_{2}$, and $\mathrm{TiO}_{2}$ are often grown or deposited with techniques that lead to lower crystal quality and higher concentrations of unintentional impurities. It has often been observed that oxide semiconductors contain large concentrations of native point defects such as vacancies and interstitials, which dominates conductivity [1]. Among oxides, the delafossite family is a rich and less explored family. $\mathrm{CuAlO}_{2}$ is one of the most extensively studied delafossite material. It is a p-type semiconductor with a wide optical bandgap and a smaller fundamental indirect bandgap, which results in better conductivity [2-5]. This is a unique property that makes $\mathrm{CuAlO}_{2}$ promising for optoelectronic and photocatalysis applications.

$\mathrm{CuAlO}_{2}$ structure: It has a rhombohedral $(R \overline{3} \mathrm{~m})$ crystal structure with space group 166 [6-8]. The primitive unit cell contains four atoms: a copper, an aluminum, and two oxygens. The conventional unit 
cell has a hexagonal structure with 12 atoms. See Figure 1.2 for the illustration of the crystal structure using the conventional unit cell and the first Brillouin zone [9] with the high symmetry points are marked on it.

$\mathrm{CuAlO}_{2}$ bandgaps: There is an agreement in reporting the direct transition, which is stated in the range of 3.4-4.0 eV [3,10-12]. However, there has been a controversy over the determination of the indirect bandgap. In some reports, the fundamental bandgap value is reported as $1.6-2.0 \mathrm{eV}[2,3,5]$, but there is no theoretical evidence to support such a low indirect bandgap value. Also, it is discussed that the bandgap of $\mathrm{CuAlO}_{2}$ should be larger than the $\mathrm{Cu}_{2} \mathrm{O}$ minimum gap $\sim 2.2 \mathrm{eV}$ because of the lower mean $\mathrm{Cu}-\mathrm{Cu}$ coordination [13]. In recent first-principle studies, the indirect bandgap value is estimated to be about 3 $\mathrm{eV}[14,15]$.

$\mathrm{CuAlO}_{2}$ for transparent conductive oxide application: It is observed and confirmed in many experimental investigations that there are some defect-dependent charge transitions; for example, the peaks at $0.9 \mathrm{eV}$ and $1.8 \mathrm{eV}$ reported by Pellicer-Porres et al. [15] are due to defect levels. The possible defects study has been performed by Scanlon et al. [16] using the first principle calculations, which confirms the mentioned defect peaks are due to $\mathrm{Cu}$ vacancy and $\mathrm{Al}$ anti-sites. These two defect levels have the smallest formation energies. It is confirmed that the conductivity of $\mathrm{Cu}_{2} \mathrm{O}$ related materials such as $\mathrm{CuAlO}_{2}$ is due to the $\mathrm{Cu}$-vacancy. Therefore, p-type conductivity in $\mathrm{CuAlO}_{2}$ is governed by band conduction with the holes thermally activated from acceptor levels or the $\mathrm{Cu}$ vacancies [17]. Because it is difficult to manipulate defects, the applicability of $\mathrm{CuAlO}_{2}$ for transparent conductive oxide is challenging [16].

$\mathrm{CuAlO}_{2}$ for Photocatalysis: The development of high-efficiency photocatalysts has been a challenge for a few decades. A potential photocatalyst should have high quantum-efficiency, be chemically stable in an aqueous environment, be durable, have a simple synthetic process, and be easy for mass production. Oxide families have attracted particular attention thanks to their chemical stability in electrolytes [18-26]. However, mostly they are transparent to the solar spectrum and consequently of little practical use. Delafossite family $\left(\mathrm{AMO}_{2}\right)$ has been one of the candidates, and a lot of studies have been done on them in recent years because of their outstanding longevity, elements abundance, much lower cost, 
and better absorbance in the solar spectrum range [21-24,26-33]. However, to date, a limited number of delafossite have been reported capable of carrying out photoelectrocatalysis, including $\mathrm{CuAlO}_{2}, \mathrm{CuLaO}_{2}$, $\mathrm{CuCrO}_{2}, \mathrm{CuYO}_{2}, \mathrm{CuFeO}_{2}$, and $\mathrm{CuRhO}_{2}$ [31]. Studies on copper and silver-based delafossites reveal that the visible light absorption of Ag delafossites is lower than copper delafossites due to a larger bandgap, although conductivities of silver delafossites are similar or lower.

There have been efforts to improve the photocatalysis activity in $\mathrm{CuAlO}_{2}$ by varying the $\mathrm{PH}$ of the aqueous environment, temperature, particle size of the powders, the addition of sacrificial hole scavengers (sulfide and sulfite), or depositing on a Fluorine tin oxide substrate with a thin Au layer to facilitate the hole transfer, etc. But the effect of alloying the B-site with a transition metal has not been studied yet, and that is going to be addressed in this thesis.

In oxide semiconductors, the effect of defects in conductivity can be experimentally studied by several experimental techniques; for instance, photoluminescence (PL) spectroscopy, temperaturedependent Hall measurements, UV-Vis-NIR absorbance spectroscopy, and electron paramagnetic resonance (EPR) are used to study the hyperfine structure of defects. While these methods are essential in the characterization of materials, they cannot conclusively and unambiguously determine the microscopic origins and mechanism of the phenomena they observe [1]. The first principle calculations are crucial for understanding the effect of various parameters such as defects, impurities, possible optical transitions, etc., in complex oxide family, including delafossite. In the following section, we will provide some background information about the modeling of $\mathrm{CuAlO}_{2}$.

\subsection{Overview of Modeling for Dilute Alloys:}

Theory and modeling can significantly enhance the process of detection, discovery, and identification of electronic structure at the material level. In particular, recent methodological advancements have made the first-principles calculations a powerful predictive tool for studying the properties of semiconductors with high accuracy. The approach, based on density functional theory (DFT), allow for a 
reliable description of the electronic structure of the electrical and optical properties of bulk materials as well as dopants and native defects, providing not only quantitative results but also a framework for studying the impact of defects on materials properties [1].

There are two main issues to be addressed to investigate and confirm the material properties computationally are transparency and conductivity. Conductivity is modeled by the phenomenological Drude theory in the IR energy range, but it is incapable of producing quantitative results at visible or UV wavelengths, where band-structure calculations play an important role [1]. Very recently, significant progress has been made with first-principles descriptions of optical absorption.

$\mathrm{CuAlO}_{2}$ electronic band structure calculations overview: Our goal is to improve optical absorption in the visible range by alloying $\mathrm{CuAlO}_{2}$ with $\mathrm{Fe}$, which can be successfully studied by the density functional theory calculations. It should be highlighted that all of the DFT calculations are based on single crystals with no intrinsic or extrinsic defects. However, the role of impurities and defects related to optical absorption through defect states is studied by DFT calculations [16]. To explain the nature of optical transitions in our experimental results in Chapter 6, we are benefiting from the defect study and the electronic band structure calculations. See Figure 1.2 that shows the schematic view of the density of state variation for parent compounds and the alloys. 
Experimental and computational investigation of the optical properties in the delafossite family, as well as, $\mathrm{CuAlO}_{2}$ has been a challenge due to their complicated structure, the intrinsic and inevitable defects, and the significant exciton energy and lattice polarization [16,17,34]. However, using thorough first principle DFT calculations, the electronic band structure of these materials is unraveled. DFT $+U$ and hybrid functional theories are the best methods to calculate the band structure because they determine the closest results to the experiment [34].

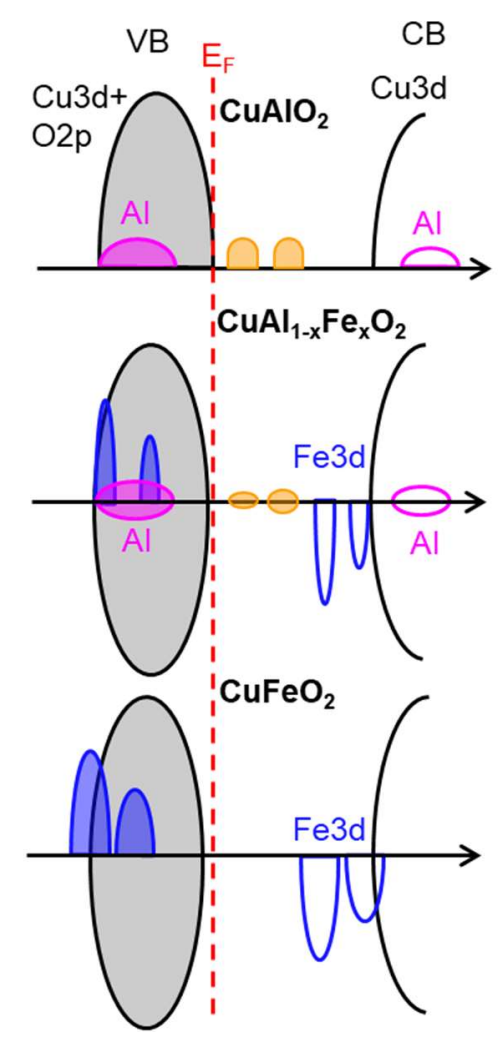

Figure 1.3: Schematic view of $\mathrm{CuAlO}_{2}, \mathrm{CuAl}_{1-x} \mathrm{Fe}_{x} \mathrm{O}_{2}$, and $\mathrm{CuFeO}_{2}$ density of states in the valance and the conduction bands. The intrinsic defects in all of the materials are present that are shown in orange.

$\mathrm{CuAlO}_{2}$ phonon calculations overview: Another useful application of the DFT in materials is to be able to predict the phonon modes that are important in various physics phenomena such as superconductivity, high entropy alloys strength, electron-phonon interaction, etc. There are various experimental techniques to measure the phonon modes and the phonon distribution, such as Raman and Fourier transform infrared (FTIR) spectroscopies, x-ray spectroscopy, neutron scattering [35]. Computational study of alloys has been a challenge due to the high computational cost and failure of 
approximation methods to explain the complex structures and compounds due to simplifications. However, there are a variety of methods to compute the phonon modes reported for alloys, which either solves the real lattice structure such as density functional phonon theory (DFPT) [36,37], finite displacement [38,39], small-displacement methods or takes advantage of modification of physical lattice parameters. A few most common approximations methods are as follows. Virtual crystal approximation (VCA) [40] is averaging over the pseudopotentials of parent materials. It is a simple and computationally efficient method. VCA method fails because the plane waves and quasiparticle approximations are used, and in the presence of the impurities, the plane wave cannot describe the alloyed system. So, this method is feasible for neighbor elements. Another common technique is a coherent potential approximation (CPA) [41], which modifies the charge density using the Green function approach, leaving the bond length and force constants unmodified. Itinerate coherent potential approximation (ICPA) [42] computes the vibrational modes with consideration of atomic mass and force among the atomic sites. Recently, there are new modifications considered to explain the phonon broadening in the alloys considering the mass and force constant fluctuations [43], first introduced by Klemens [44]. Also, the new report on modifying the vibrational wave function in the presence of impurities can explain the phonon modes in more agreement with experimental results $[45,46]$. When magnetic elements are involved, the computational load becomes twice heavier. In addition, for the dilute alloys, the calculation of the vibrational mode becomes much more massive. The calculation of vibrational frequencies (phonon spectra) in dilute anisotropic alloys is not a simple undertaking and the successful methodologies are not well developed. For this reason, our original approach started with the accurate prediction of the lattice structure of the dilute alloys. To determine lattice structure of these dilute alloys we used a disordered supercell. However, to continue and use these results to determine the phonon spectra, especially for dilute anisotropic alloys, is too computationally demanding. In principle, the calculation of the vibrational frequencies can be done based on the supercell results; however, these calculations are only tractable in the case of the parent structures, $\mathrm{CuAlO}_{2}$ and $\mathrm{CuFeO}_{2}$, at the end points. Again, for the alloys, especially the dilute alloys, the very large supercells make the calculations too computationally demanding. For these reasons, we developed a new effective-medium 
approach using a weighted dynamical matrix (WDM), based on modifying the masses and interatomic force constants after calculation of Hellmann-Feynman forces [47] for parent compounds $\mathrm{CuAlO}_{2}$ and $\mathrm{CuFeO}_{2}[48-51]$ (details are in 2.2.2).

\subsection{Goal of the Thesis:}

I studied the effect of alloying $\mathrm{Fe}$ to $\mathrm{CuAlO}_{2}$ delafossite on its physical properties. The alloying can vary the optical, magnetic, vibrational, and many other properties of $\mathrm{CuAlO}_{2}$. The theories suggest that by replacing Fe in Al sites, the inversion symmetry of the rhombohedral $(\mathrm{R} \overline{3} \mathrm{~m})$ lattice will be broken, and the forbidden transitions become allowed [4]. Also, we expect from the DFT electronic band structure calculations for $\mathrm{CuFeO}_{2}$ that $\mathrm{Fe}-3 \mathrm{~d}$ states form in the bandgap of $\mathrm{CuAlO}_{2}$ [52]. These two variations may lead to lowering bandgap value and increases the absorption in the optical spectrum and better photocatalytic activity.

Furthermore, by doping $\mathrm{CuAlO}_{2}$ with transition metal ions, it is predicted that the resulting alloy becomes a dilute magnetic semiconductor suitable for spintronic applications [53]. The question of whether $\mathrm{CuAl}_{1-x} \mathrm{Fe}_{x} \mathrm{O}_{2}$ is a room temperature ferromagnet, as some recent papers have reported, needs a thorough study that we are presenting here.

Moreover, the study of the variation of vibrational modes by alloying $\mathrm{CuAlO}_{2}$ with $\mathrm{Fe}$ is essential because $\mathrm{CuAlO}_{2}$ has an indirect fundamental bandgap, which is due to phonon-assisted optical transitions. On the other hand, alloying leads to chemical disorder in the lattice and causes phonon scattering [43]. Understanding the vibrational properties of $\mathrm{CuAl}_{1-x} \mathrm{Fe}_{x} \mathrm{O}_{2}$ alloys is key to understanding the variation of optical (photon-photon interaction) and electronic (electron-phonon interaction) of properties with $\mathrm{Fe}$ concentration. To that end, we investigate the structural, electronic, magnetic, and optical properties of Fedoped $\mathrm{CuAlO}_{2}[48,54]$. 


\subsection{Organization of Dissertation:}

The rest of the dissertation is organized as follows: In Chapter 2, The sample preparation using solid-state reaction for $\mathrm{CuAl}_{1-x} \mathrm{Fe}_{x} \mathrm{O}_{2}$ alloys for $x=0$ to 1 is described and is optimized to produce the least amount of spinel phase. The Rietveld refinement is used to quantify the phases. Various methods are performed to characterize the powder samples, such as x-ray diffraction (XRD) for crystallography, $\mathrm{x}$-ray photoelectron spectroscopy (XPS) for elemental and chemical study, scanning electron microscopy for the size and morphology, and energy dispersive spectroscopy for the elemental characterization. In Chapter 3, a summary of the density functional theory and various DFT methodologies are described. The challenges of the electronic band structure and phonon calculations for the alloys are explained. The details of the DFT calculations performed for the lattice parameters, electronic band structure, and phonon dispersion are explained. Also, for the vibrational calculations of the non-isoelectronic dilute anisotropic alloys, a new weighted dynamical matrix is introduced. In Chapter 4, the temperature and magnetic field dependent measurements are performed on all the samples and the theory to fit the data, determining the type of interaction, availability of magnetic phase transitions and magnetic order, and the concentration and type of magnetic ions are explained. The high-resolution hysteresis measurements are performed to investigate the possibility of the room temperature ferromagnetism. The electron spin resonance (ESR) at room temperature is explained to investigate to determine the magnetic ion properties. In Chapter 5, the theory of vibrational modes in the crystal structures is explained. Then, the phonon dynamics for the alloys are measured using the Raman and FTIR spectroscopies, and the weighted dynamical matrix computational results are compared with the experimental results. In Chapter 6, the methodology for the measurement of the optical absorbance and the Kobelka-Munk theory to find the absorption and the Tauc equation are explained in summary. The FTIR, UV-Vis, and UV-Vis-NIR measurements that are performed with various instruments are described. The electronic band structure calculations are performed by GGA+U, and the unfolded band structure is used to explain the optical features observed in the optical absorbance spectra. 


\section{Chapter 2}

\section{Preparation of Samples and their Structural Characterization}

\subsection{Introduction:}

In this chapter, procedures used for the solid-state synthesis and the optimization to prepare $\mathrm{CuAl}_{1-}$. ${ }_{x} \mathrm{Fe}_{x} \mathrm{O}_{2}$ alloys for $x=0.0,0.01,0.05,0.1$, and 1.0 are described. Results from characterizations of the samples using x-ray crystallography, including Rietveld refinement, elemental, and chemical analysis, are also presented. The below flow chart shows the steps to synthesize and characterize the powders.

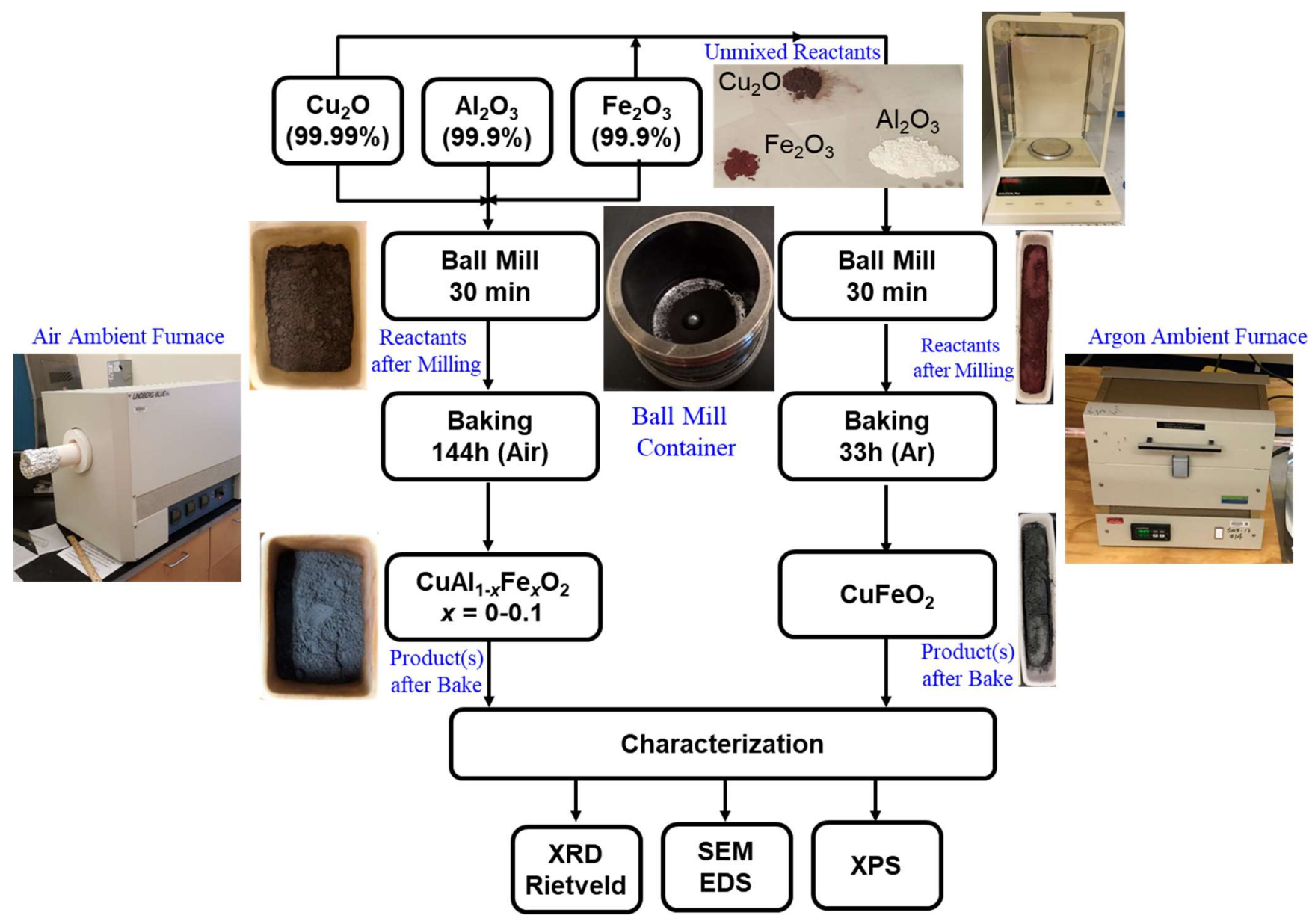

Figure 2.1: Flowchart of the synthesis of $\mathrm{CuAl}_{1-x} \mathrm{Fe}_{x} \mathrm{O}_{2}$ by the solid-state reaction of stoichiometric mixture of $\mathrm{Cu}_{2} \mathrm{O}, \mathrm{Al}_{2} \mathrm{O}_{3}$, and $\mathrm{Fe}_{2} \mathrm{O}_{3}$ and the characterization methods performed and explained in this chapter. 


\subsection{Overview of Procedures for Synthesis and Growth of Delafossites}

\subsubsection{Synthesis of Polycrystalline Powders:}

The delafossite $\left(\mathrm{A}^{+} \mathrm{M}^{3+} \mathrm{O}_{2}\right)$ powders are commonly synthesized by solid-state reaction. A controlled growth condition is necessary to grow single-phase powders. Amrute et al. [55] investigated the influence of various parameters, such as temperature, atmosphere, annealing time, and ball milling of precursors, on the solid-state synthesis of cuprous delafossites with different trivalent cations $\left(\mathrm{M}^{3+}\right)$. We benefit from this detailed investigation for the starting procedure of the optimized temperature, mechanism, and kinetics of their formation to produce single-phase materials. A summary of their findings is given below.
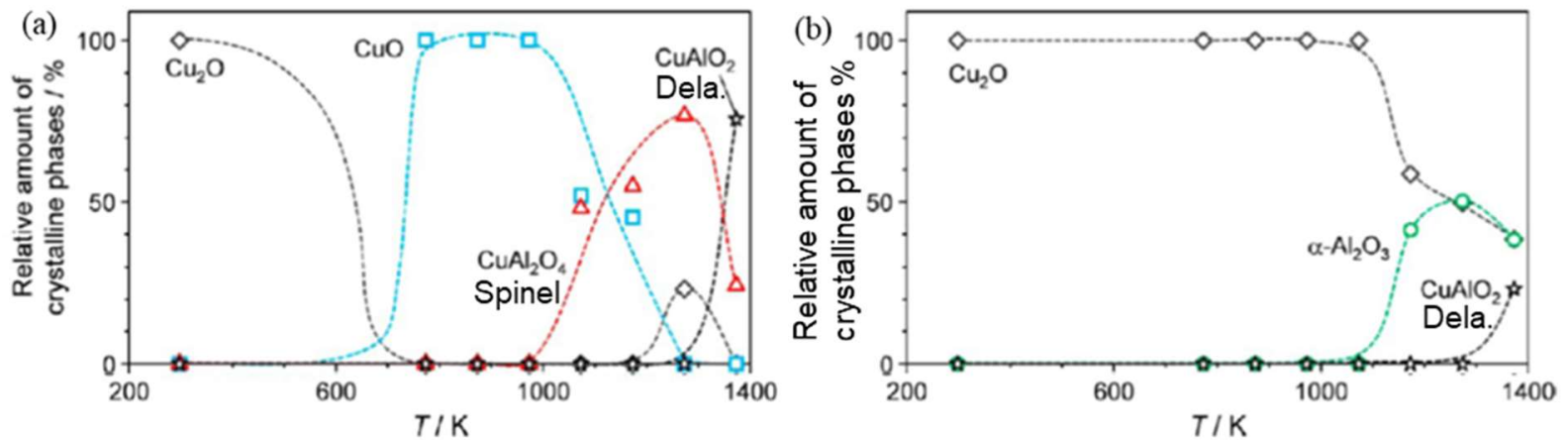

Figure 2.2: Evolution of the crystalline phases during the synthesis of $\mathrm{CuAlO}_{2}$ in air (a) and $\mathrm{N}_{2}$ (b) as determined from the in situ XRD analysis measured by Amrute et al. [55]

Considering the $\mathrm{Cu}_{2} \mathrm{O}+\mathrm{Al}_{2} \mathrm{O}_{3}$ mixture in air, the evolution of phases is as follows (see Figure 2.2 (a) and (b)): from room temperature to $773 \mathrm{~K}, \mathrm{Cu}_{2} \mathrm{O}$ was depleted, and $\mathrm{CuO}$ was formed; from 973 to 1273 $\mathrm{K}, \mathrm{CuO}$ disappeared, and the mixed spinel $\mathrm{CuAl}_{2} \mathrm{O}_{4}$ phase developed to a significant extent; a moderate amount of $\mathrm{Cu}_{2} \mathrm{O}$ reformed at $1273 \mathrm{~K}$; from 1273 to $1373 \mathrm{~K}$, the spinel and $\mathrm{Cu}_{2} \mathrm{O}$ were partially and fully depleted, respectively, and delafossite $\mathrm{CuAlO}_{2}$ was produced. Accordingly, the formation of the $\mathrm{Cu}-\mathrm{Al}$ delafossite involves several steps: $(i)$ the oxidation of $\mathrm{Cu}_{2} \mathrm{O}$ to $\mathrm{CuO}$, in line with the TGA data (Figure 2.2); (ii) the reaction of the latter with $\mathrm{Al}_{2} \mathrm{O}_{3}$ to form the spinel $\mathrm{CuAl}_{2} \mathrm{O}_{4}$; (iii) the reduction of the unconverted $\mathrm{CuO}$ back into $\mathrm{Cu}_{2} \mathrm{O}$; and finally, (iv) the reaction of $\mathrm{Cu}_{2} \mathrm{O}$ and the spinel to form $\mathrm{CuAlO}_{2}$. Their XRD study confirms the formation mechanism of delafossite $\mathrm{CuAlO}_{2}$, which illustrates the same intermediate formation of the spinel phase, and the subsequent reaction with $\mathrm{CuO}\left(\right.$ rather than $\mathrm{Cu}_{2} \mathrm{O}$ ) to produce delafossite. If the same procedure is done in inert $\mathrm{N}_{2}$, the $\mathrm{Cu}_{2} \mathrm{O}$ did not change up to $1273 \mathrm{~K}$, and the alumina 


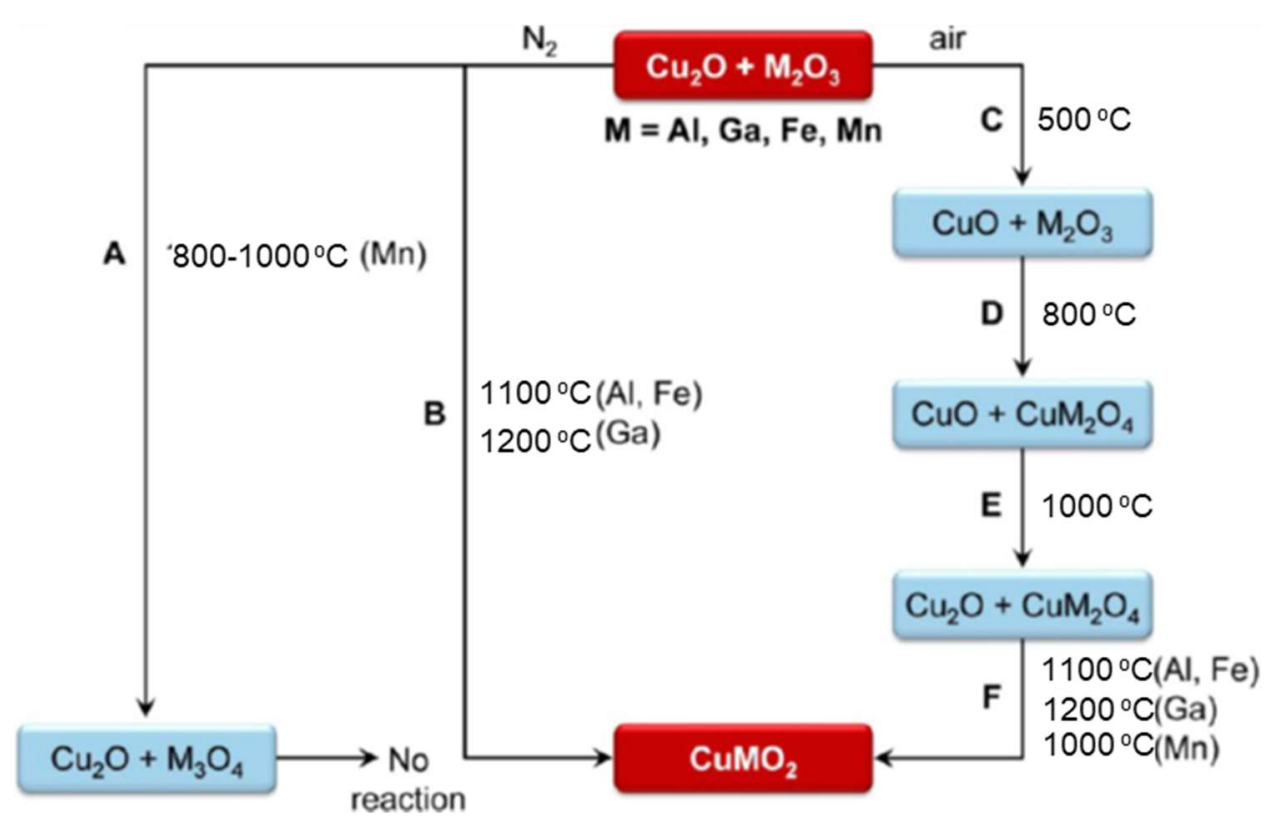

Figure 2.3: Steps involved in the formation of $\mathrm{CuAlO}_{2}$ by the solid-state reaction of the single oxides. Four-step mechanism for the delafossites forming in air above $1273 \mathrm{~K}$, Amrute et al.[55]

was not consumed in the reaction with $\mathrm{CuO}$. The formation of $\mathrm{CuAlO}_{2}$ took place at $1373 \mathrm{~K}$ by the solidstate reaction of $\mathrm{Cu}_{2} \mathrm{O}$ and $\mathrm{Al}_{2} \mathrm{O}_{3}$. Figure 2.3 shows a schematic of the synthesis procedure. They compared the usage of $\mathrm{CuO}$ with $\mathrm{Cu}_{2} \mathrm{O}$ in the solid-state reaction formation temperature to produce $\mathrm{CuAlO}_{2}$. The TGA-DTA analysis did not show significant differences.

In our synthesis, $\mathrm{Cu}_{2} \mathrm{O}$ is used as the copper source in the rest of the study. Furthermore, the ball milling activates the precursors by changing the particle size (grinding) and better mixing, which led to faster kinetics of delafossite production. In Figure 2.4, the percentage of $\mathrm{CuAlO}_{2}$, which is calcinated (the process of heating a substance to a high temperature but below its melting point to bring about a thermal decomposition.) for $10 \mathrm{~h}$, is plotted. Delafossite phase formation is linearly scaled with the duration of ball milling. Accordingly, longer milling times could be applied to reduce the required dwelling time at high temperatures to achieve pure phase delafossite. 
In our work, the samples are prepared by 30 minutes of ball milling of a stoichiometric mixture of $\mathrm{Cu}_{2} \mathrm{O}, \mathrm{Al}_{2} \mathrm{O}_{3}$, and $\alpha-\mathrm{Fe}_{2} \mathrm{O}_{3}$ before sintering the powders in the air at $1100{ }^{\circ} \mathrm{C}$. However, only $\mathrm{CuFeO}_{2}$ powder is synthesized at $1000^{\circ} \mathrm{C}$ in $\operatorname{Ar}[7,8,48,56]$.

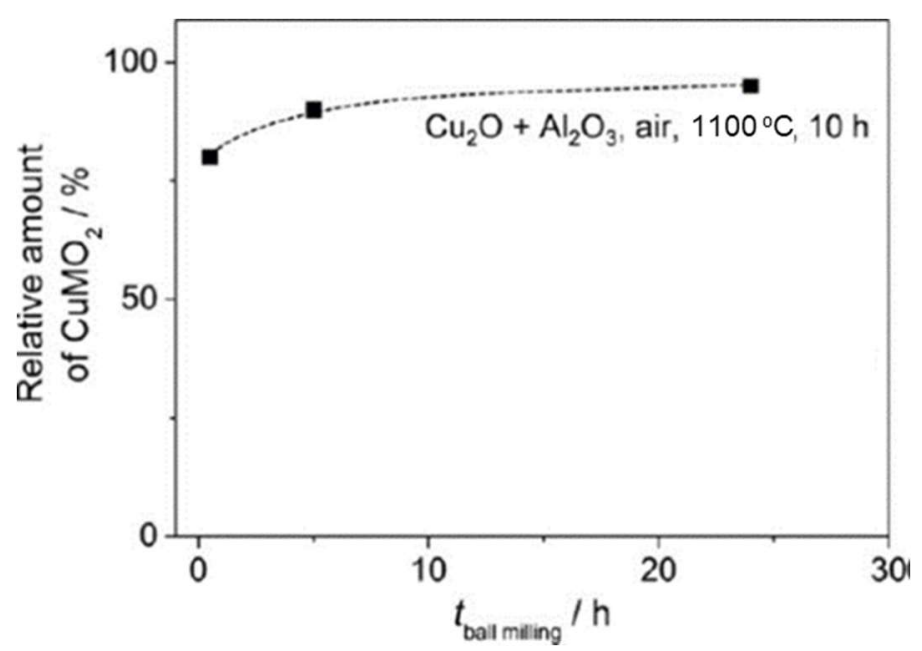

Figure 2.4.: Amount of $\mathrm{CuAlO}_{2}$ formed in air after ball milling of their precursors for increasing times, measured by Amrute et al.[55]

\subsection{X-ray diffraction analysis:}

\subsubsection{Particle Size Analysis:}

When crystallite size is less than about $100 \mathrm{~nm}$, appreciable broadening in the $\mathrm{x}$-ray diffraction lines will occur. This broadening may correspond to the actual size of the particles. The observed line broadening, which is estimated by the full width at half maximum of the peaks, can be used to estimate the average size. In the simplest case in which the particles are stress-free, the size can be determined from a single diffraction peak $[57,58]$. In this case, Scherrer's equation is used:

$t=\frac{K \lambda}{\beta c o \quad B}$

Where $t, \lambda, \theta_{\mathrm{B}}$, and $\beta$ correspond to crystallite size, $\mathrm{x}$-ray wavelength in $\AA$, the Bragg's peak position in radian and full width at half maximum of Bragg's peaks. The proportionality constant, K, depends on how the width is determined, the shape of the crystal, and the size distribution. Common values for $\mathrm{K}$ are 0.94 for spherical crystals with cubic symmetry and 0.89 for the integral breadth of spherical crystals with cubic symmetry. Therefore, both cases are close to 0.9 . On the other hand, in those cases where stress may 
be present, the Williamson-Hall method involving several diffraction peaks is required [59]. In our calculations, Scherrer's method is used, as the stress is not significant, which is concluded from the calculation of particle size by the Williamson-Hall method.

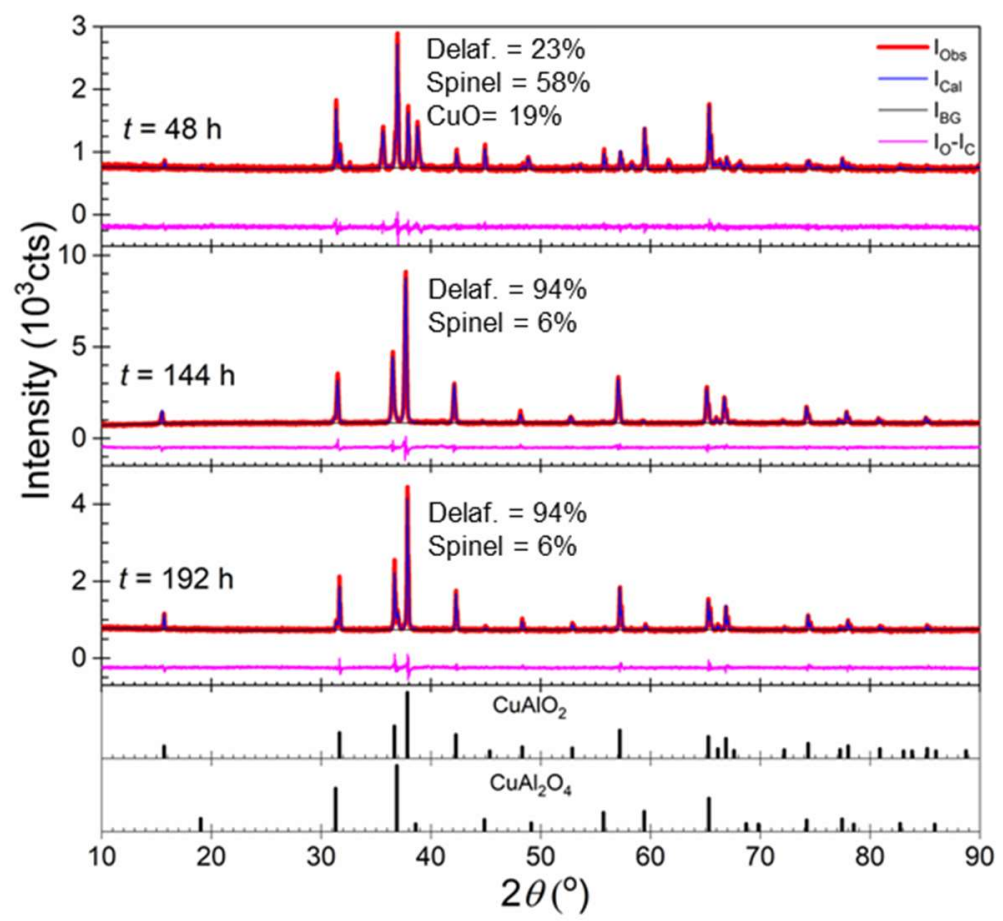

Figure 2.5: XRD spectra and their Rietveld refinements for $\mathrm{CuAlO}_{2}$ samples baked for three $48 \mathrm{~h}, 144 \mathrm{~h}$, and 192 h, cyan, blue lines used for experimental data, nonlinear background, and Rietveld refinement, respectively.

\subsubsection{Rietveld Refinement:}

The Rietveld refinement $[60,61]$ for powder-diffraction data is a multi-parameter curve-fitting procedure used to quantify the $\%$ by weight of the different crystalline phases present in a sample [60]. Many diffraction peaks or lines of the diffraction pattern for each phase are considered so that overlapping lines from different patterns are not a problem. A fitted spectrum is determined by minimizing the $\chi$-squared difference between the fit and experimental spectra based on the known crystal structure parameters for each phase and fitting parameters such as weight percent, crystallite size, and strain for each phase. This calculated spectrum includes the contributions from the sharp Bragg peaks of the crystalline components of the sample and the contribution of the smooth background that underlies the peaks. 


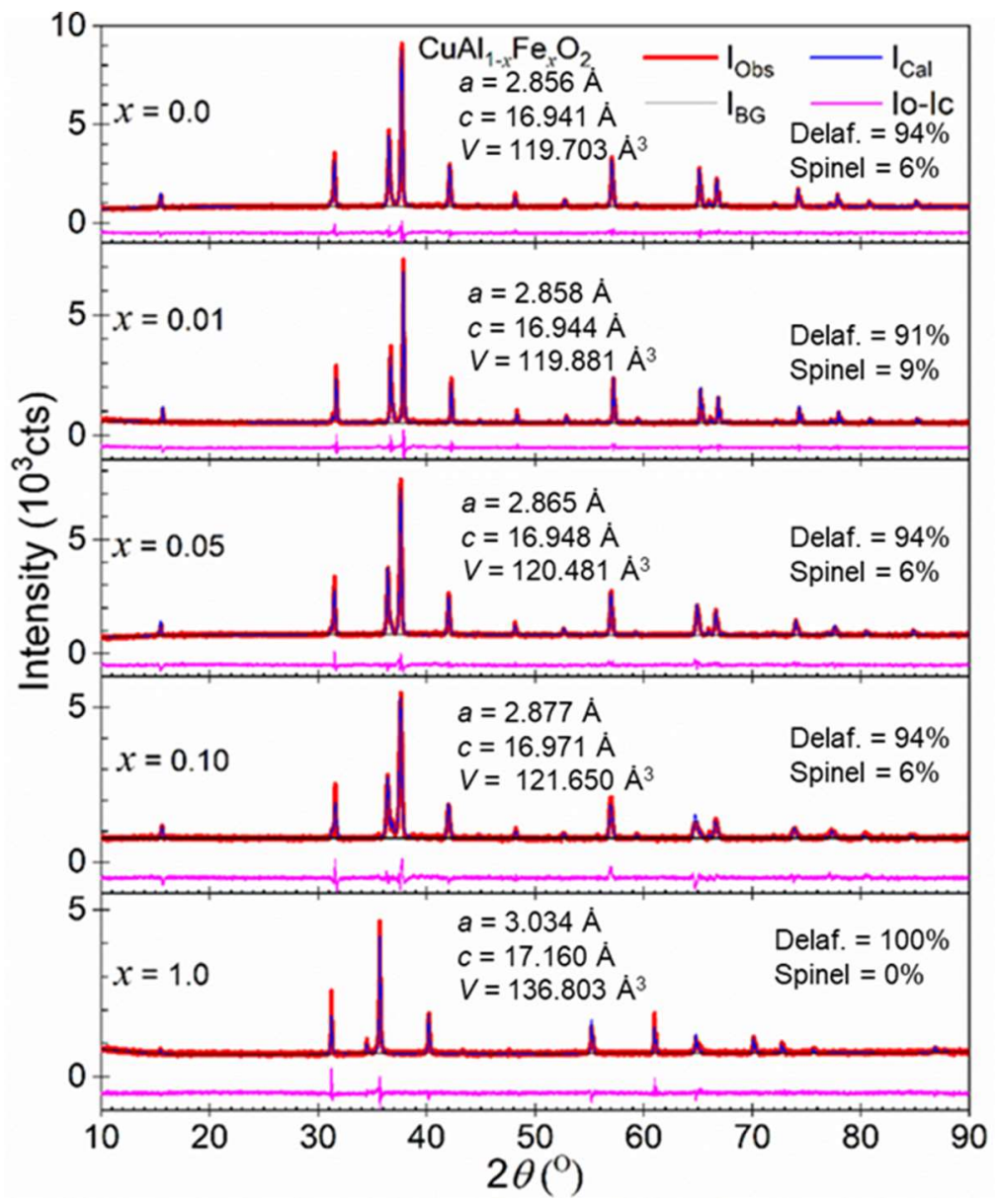

Figure 2.6: XRD spectra and their Rietveld refinements for $\mathrm{CuAl}_{1-x} \mathrm{Fe}_{x} \mathrm{O}_{2}$ samples with $x=0,0.01,0.05$ and 0.1 . Red, cyan, blue lines used for experimental data, nonlinear background, and Rietveld refinement, respectively. Percentage quantification of delafossite and spinel phases are shown.

Polycrystalline samples of $\mathrm{CuAl}_{1-x} \mathrm{Fe}_{x} \mathrm{O}_{2}$, for $x=0.0,0.01,0.05,0.1$, and 1.0 are prepared by solidstate reaction of a stoichiometric mixture of $\mathrm{Cu}_{2} \mathrm{O}(99.99 \%), \mathrm{Al}_{2} \mathrm{O}_{3}(99.9 \%)$, and $\mathrm{Fe}_{2} \mathrm{O}_{3}(99.9 \%)$. Two recipes were tested for the mixing process of the $\mathrm{CuAl}_{1-x} \mathrm{Fe}_{x} \mathrm{O}_{2}$ powders. First, the samples of precursor oxides in stoichiometric ratios were ball milled for 30 minutes and then baked at $1100{ }^{\circ} \mathrm{C}$ in air. In this step, the necessary time for the purest delafossite phase is optimized $[7,8,48]$. The $\mathrm{x}$-ray diffraction was done using a PANalytical X'Pert Pro x-ray Diffractometer (XRD) with a Cu-K $\mathrm{K}_{\alpha}$ source $(\lambda=1.542 \AA)$. In Figure 2.5, the ex-situ XRD for 48, 144, $192 \mathrm{~h}$ baking times are shown. The Rietveld refinement by the HighScore Pro software is used to quantify the possible phases using XRD spectra. Figure 2.5 shows the experimental, refined, and background data, $I_{\mathrm{O}}, I_{\mathrm{C}}$, and $I_{\mathrm{BG}}$, respectively. The $\chi$-squared, goodness of fit (GOF) values are tabulated for each sample. The $\chi$-squared values and the difference between experimental and calculated 
spectra $\left(I_{\mathrm{O}}-I_{\mathrm{C}}\right)$ show very good agreement. According to the Rietveld results, the purest delafossite phase is achieved at $144 \mathrm{~h}$. Second, the starting compounds are mixed by ball mill for 30 minutes without acetone and calcinated in the tube furnace at $1100^{\circ} \mathrm{C}$ for $144 \mathrm{~h}$ in air. The XRD patterns of the samples of $\mathrm{CuAl}_{1-}$ ${ }_{x} \mathrm{Fe}_{x} \mathrm{O}_{2}$ with $x=0.0,0.01,0.05$, and 0.1 were prepared under our optimum procedure (baking for 144 hours at $1,100^{\circ} \mathrm{C}$ in the air) are shown in Figure 2.6. It is evident that the $x=0.01$ sample does have more amount of the spinel phase, although the concentration of the spinel phase decreases considerably in the $x=0.05$ and 0.1 samples and. This issue is included in our analysis of the magnetic data. It is noted that the expected electronic state of $\mathrm{Fe}$ in $\mathrm{CuAl}_{1-x} \mathrm{Fe}_{x} \mathrm{O}_{2}$ is $\mathrm{Fe}^{3+}$ if it substitutes for $\mathrm{Al}^{3+}$. With our careful analysis of the phases, we showed that there is about $5 \%$ of the spinel phase present in the powders. The alloys synthesized powders XRD spectra along with the Rietveld refinements are plotted in Figure 2.6. However, the same recipe does not work for $\mathrm{CuFeO}_{2}$. For $x=1.0, \mathrm{CuFeO}_{2}$ is synthesized by 30 minutes of ball-milled starting materials in $\mathrm{Ar}$ ambient at $1000^{\circ} \mathrm{C}$ for $30 \mathrm{~h}$ in the tube furnace. The XRD and the Rietveld results are showing that the single-phase delafossite $\mathrm{CuFeO}_{2}$ powder is produced (see Figure 2.6). The lattice parameters are given in Figure 2.6 based on the Rietveld refinement. 


\subsubsection{Variation of Lattice Parameters by increasing Fe concentration:}

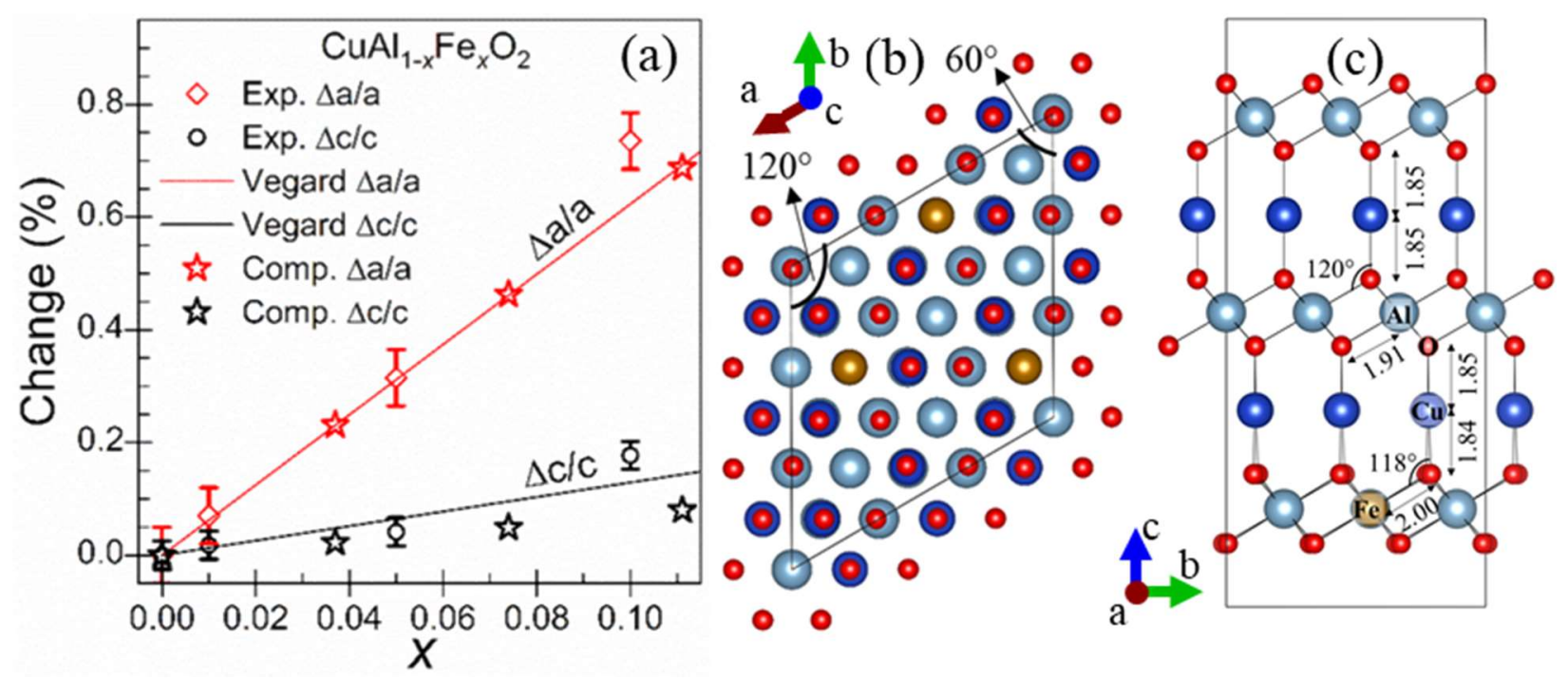

Figure 2.7: (a) Experimental data with error bars for percentage change of $a$ and $c$ vs. $x$ for $\mathrm{CuAl}_{1-\mathrm{x}} \mathrm{Fe}_{\mathrm{x}} \mathrm{O}_{2}$ (with $x=$ 0, 0.01, 0.05, and 0.10). Computational lattice parameters with PBEsol+ $U$ by supercell of $\mathrm{CuAl}_{1-x} \mathrm{Fe}_{\mathrm{x}} \mathrm{O}_{2}$ are also shown. Lines show the fit to Vegard's law to the experimental data (b) and (c) show the minimum energy configuration for $x=0.11$ along, respectively, $\mathrm{c}$ and a-axis.

The observed percentage changes in the lattice parameters $a, c$, and volume $V$ with an increase in $x$ determined from the XRD patterns are plotted in Figure 2.7 (a). The \% changes in $a$ are larger than those in $c$, confirming the substitution of the larger $\mathrm{Fe}^{3+}$ for $\mathrm{Al}^{3+}$. The solid black lines in Figure 2.7 (a) show the fit to Vegard's equation, using $a_{\mathrm{CuAlO}_{2}}=2.86 \AA$ and $a_{\mathrm{CuFeO}_{2}}=3.04 \AA$, and $c_{\mathrm{CuAlO}_{2}}=16.96 \AA$, and $c_{\mathrm{CuFeO}_{2}}=$ $17.17 \AA$. Vegard's equations for $a$ and $c$ are as follow $[62,63]$ :

$a_{\mathrm{CuAl}_{1-x} \mathrm{Fe}_{x} \mathrm{O}_{2}}=(1-x) \cdot a_{\mathrm{CuAlO}_{2}}+x \cdot a_{\mathrm{CuFeO}_{2}}$

$c_{\mathrm{CuAl}_{1-x} \mathrm{Fe}_{x} \mathrm{O}_{2}}=(1-x) \cdot c_{\mathrm{CuAlO}_{2}}+x \cdot c_{\mathrm{CuFeO}_{2}}$

The samples have rhombohedral delafossite structure, and there is a lattice expansion associated with replacing $\mathrm{Al}$ with $\mathrm{Fe}$, which shows up as the peaks shift to lower angles. The calculated $a$ and $c$ parameters are $a=2.86 \AA$ for $x=0$ and $a=2.88 \AA$ for $x=0.10$ representing $0.70 \%$ increase in $a$ lattice parameter. Likewise, $c$ lattice parameter increases from $c=16.96 \AA$ for $x=0$ to $c=16.97 \AA$ for $x=0.10$ showing $0.06 \%$ increase. Therefore, the increase in $a$ is more prominent than that in $c$. The conventional unit cell volume changes from $V=120.1 \AA$ for $x=0$ to $V=121.5 \AA$ showing an expansion of $1.2 \%$. A 


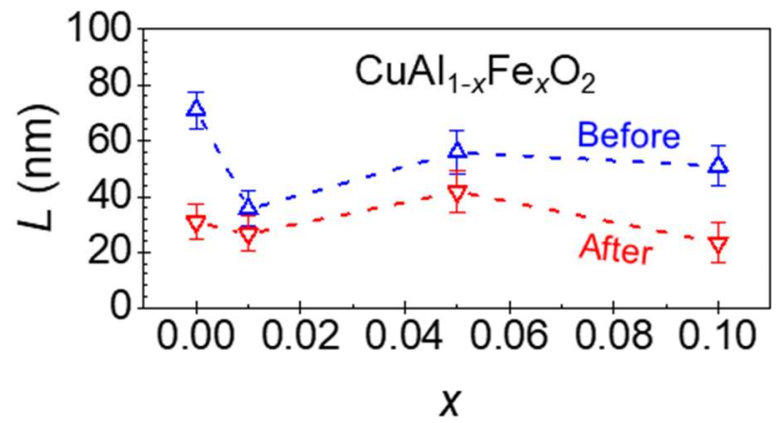

Figure 2.8: The plot shows changes in the crystallite size $(L)$ for samples before and after ball milling, as extracted from Scherrer analysis.

brief summary of the structural properties was reported in our recent publications to aid in our interpretation of Raman and FTIR results on the $\mathrm{CuAl}_{1_{-x}} \mathrm{Fe}_{x} \mathrm{O}_{2}$ samples. The XRD spectra of all four samples are refined by the Rietveld method, and the change in the lattice parameters $a$ and $c$ are calculated from the values resulted from the refinement. By substituting $\mathrm{Fe}$ for $\mathrm{Al}$ as a $\mathrm{Fe}^{3+}$ ion, the rhombohedral symmetry is unchanged, but the lattice expands with the relative expansion along the $a$-axis being $\sim 6$ times larger than that along the c-axis. Figure 2.7 shows the variation of the lattice parameters a and $\mathrm{c}$ with a change in Fedoping. The solid lines show the Vegard's law fit. The DFT calculations are done on the disordered supercells of $\mathrm{CuAl}_{1-x} \mathrm{Fe}_{x} \mathrm{O}_{2}$ for $x=0.0,0.04,0.08$, and 0.11 . See Chapter 3 for the computational details. The disordered supercell for $x=0.11$ along the $a$ - and $c$-axes are shown in Figures 2.7 (b) and 2.7 (c). Our computational results show good agreement with the experimental data and fit Vegard's law $[64,65]$. Small deviations from Vegard's law maybe since our calculations are done at $0 \mathrm{~K}$, whereas the experiments are done at room temperature. Bond lengths are marked in Figure 2.7 (c). These results imply that the substitution of $\mathrm{Fe}^{3+}$ for the smaller $\mathrm{Al}^{3+}$ (ionic radii 0.065 and $0.054 \mathrm{~nm}$, respectively) introduces localized strain to the lattice, and the $\mathrm{M}-\mathrm{O}(\mathrm{M}=\mathrm{Al}, \mathrm{Fe})$ bond lengths increases with higher Fe doping. The bonds around substituted Fe atoms become distorted, and the $\mathrm{M}-\mathrm{O}$ bond length increases ( $\mathrm{Al}-\mathrm{O}$ and $\mathrm{Fe}-\mathrm{O}$ bonds are 0.191 and $0.200 \mathrm{~nm}$, respectively); whereas, the $\mathrm{Cu}-\mathrm{O}$ bond length decreases from $0.185 \mathrm{~nm}$ to 0.184 $\mathrm{nm}$ near the dopant. These changes in the bond lengths affect the lattice parameters and vibrational modes.

After synthesis of the powders was ball-milled to make the crystallite size more evenly distributed. In Figure 2.8, the crystallite size of the as-prepared samples before and after the samples were ball-milled 
for two hours in 30 minutes steps are plotted versus $x$. This crystallite size was determined from the XRD patterns using Scherrer analysis. This ball-milling step was intended to make the crystallite size of our powders consistent. This analysis shows the expected decrease in the crystallite size $L$ for the doped samples after ball-milling [7]. The magnetic measurements reported in Chapter 4 were done on the ball-milled samples.

\subsection{Elemental Analysis by Scanning Electron Microscopy (SEM):}

\subsubsection{SEM Overview:}

(a)

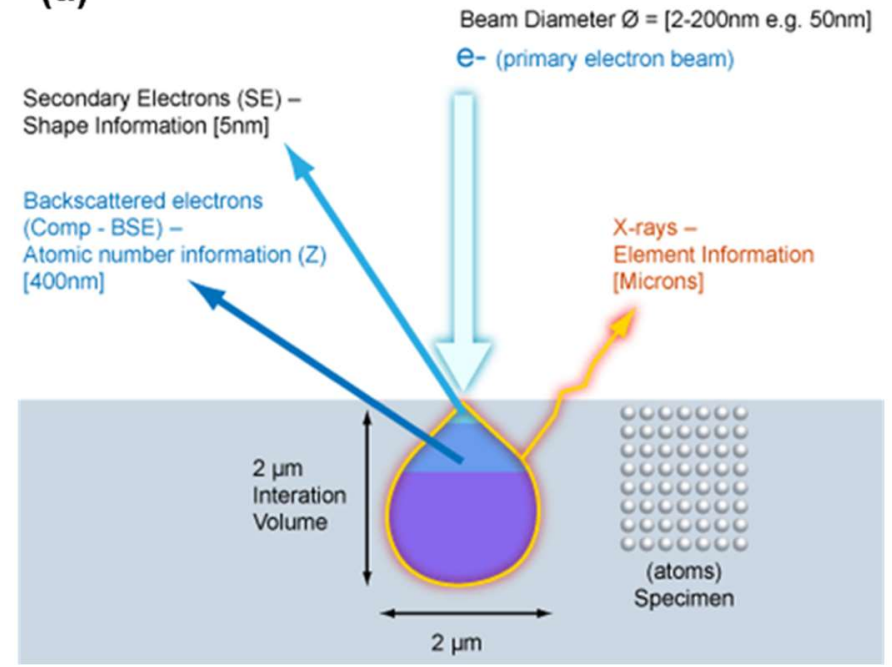

(b)

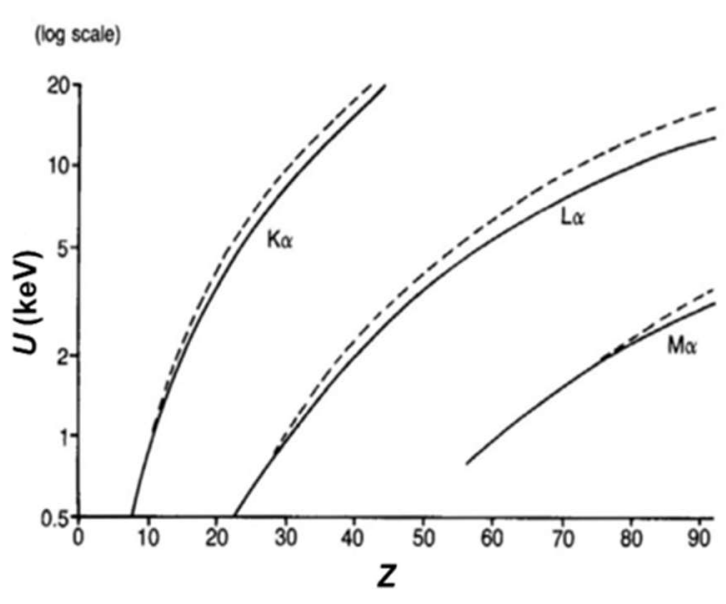

Figure 2.9: (a) shows the interaction volume of the electron beam and the sample and the possible phenomena arising from this interaction. (b) shows the dependence of excitation energy edges vs. atomic number which based on this relationship the acceleration voltage can be selected.

When the primary beam of electrons incidents on a sample, it penetrates the specimen and interacts with the atoms inside the interaction volume, see Figure 2.9 (a). The size of the interaction volume depends on the accelerating voltage of the primary electron beam and the mean atomic number (or density) of the sample. The interaction volume increases for larger accelerating voltage and decreases for samples with a higher mean atomic number. Various types of scattering can happen, and a wide variety of information can be extracted from scattered beams such as topography, elemental composition, even crystallography, phase, etc., with electron backscattered diffraction. Specific detectors are needed to obtain the information that can collect the associated scattered electrons or x-ray. In this work, the surface topography and the elemental 
composition are measured among various types of information that can be achieved by using scanning electron microscopy. Here is a summary of the secondary electron microscopy (SEM) and energy dispersive spectroscopy (EDS) that can provide information about the surface and elemental composition and the discussion of the results of the measured sample $[66,67]$.

In the SEM, some low energy electrons from the topmost surface of the sample mobilize through inelastic scattering by the primary incident beam and overcome the surface energy barrier and escape from the sample. These electrons are called secondary electrons, which is relatively insensitive to the target atomic number and is dependent on the incident beam energy. Although sample electrons mobilize throughout the interaction volume, their escape probability from the surface of the sample decreases sharply with depth, which is $\sim 10 \mathrm{~nm}$. Hence, secondary electrons are useful in studying the surface characteristics of the sample.

The electron microprobe uses x-ray spectrometry to identify and measure the concentration of elements in the microscopic volumes of the specimen. The signals detected from raster scanning the surface are used to obtain high resolution scanning electron images, in which x-ray maps show the spatial distribution of elements. Characteristic x-rays generated from an interaction volume in a single spot are used to obtain a complete quantitative elemental analysis. Sample preparation is minimal, but a wellpolished surface is required for accurate quantitative analysis. The typical range of x-ray energies analyzed in the electron microscopes is between 0.12 and $10 \mathrm{keV}$. The elemental determination is performed by the characteristic x-ray energies. In the inelastic scattering of electron beam case, strongly bound inner shell electrons probably are ejected from the target atoms if the beam energy is higher than the critical excitation energy of the target atomic shell. Subsequent relaxation of the atom from its excited state, which involves the transfer of an outer shell electron to the ionized inner shell, leads to the generation of a characteristic $\mathrm{x}$ ray. The x-ray may be emitted, or it may be absorbed and causes the ejection of another outer shell electron, which is called Auger electron. The fraction of characteristic x-rays yields or fluorescent yield for a specific 
shell increases with the atomic number, while the Auger yield decreases. Characteristic x-rays energies are associated with the following energies:

- $\mathrm{K}_{\alpha}$ : a vacancy in $\mathrm{K}$-shell is filled by a transition from L-shell

- $\mathrm{K}_{\beta}$ : a vacancy in $\mathrm{K}$-shell is filled by a transition from M-shell

- $\quad \mathrm{L}_{\alpha}$ : a vacancy in $\mathrm{L}-$ shell is filled by a transition from M-shell

- $\mathrm{M} \alpha$ : when a vacancy in M-shell is filled by a transition from $\mathrm{N}$-shell

To calculate the concentration of the elements, one should take to account the necessary corrections, which are (i) the atomic number correction (Z), (ii) absorption correction (A), and (iii) fluorescence correction (F), which is well known as ZAF. The matrix correction depends on the composition of the specimen. Hence, the composition is unknown before the analysis; the ZAF factors are also unknown. Therefore, the atomic concentration is determined through iteration. Two main factors, the accelerating voltage value, and the take-off angle play a critical role in decreasing the correction values. (i) The relation between atomic number correction and accelerating voltage (U) is complex, although in general, decreases very slowly with an increase in U. (ii) Part of the x-rays generated at depth are not emitted and are absorbed in the specimen by interacting with other specimen atoms, which may become ionized as a result. Therefore, the absorption is maximum when the energy of the x-ray is slightly above the critical excitation energy of the target atomic shell. Since high $U$ values require large absorption corrections, a low $\mathrm{U}$ is preferable, and the larger is the take-off angle, the lower is the absorption coefficient. (iii) x-ray fluorescence occurs because of the photoelectric absorption of primary x-rays as the target atom relaxes from an excited state. Since x-rays can travel longer distances than electrons in matter, the range of fluoresced x-rays is also greater ( $\sim 10$ times) than the primary electron interaction range. The secondary $\mathrm{x}-$ ray interaction volume may be as much as 1000 times greater than the primary interaction volume. Therefore, a high take-off angle and lower accelerating voltage are preferable to minimize the ZAF. 
However, most electron microprobes have fixed take-off angles but a variable accelerating voltage to minimize the ZAF correction [66].

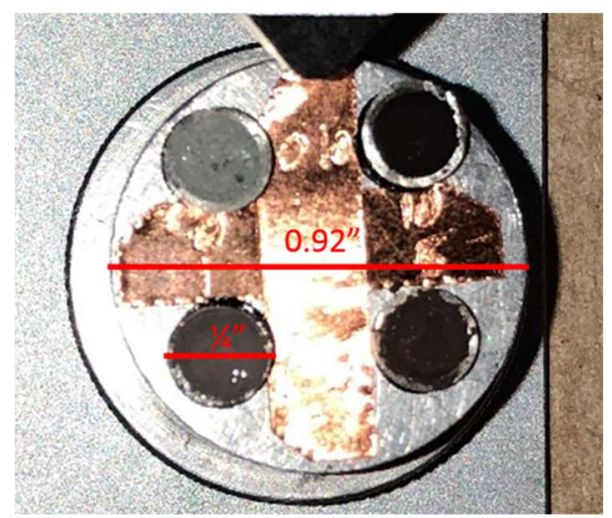

Figure 2.10: shows four samples in the titanium crucible and attached to a general sample holder. The copper tape is used to mark and facilitate the sample inside the SEM.

The accelerating voltage needs to be determined based on the contributing atoms. See Figure 2.9 (b), which shows the relationship between the atomic numbers and $U$. $U$ should not be selected much higher than the critical excitation energy to minimize the ZAF.

\subsection{Delafossite Alloys Topography and Elemental Characterization:}

The elemental characterization of the samples was performed using an EDAX system on a Hitachi S-4700 Scanning Electron Microscope, respectively, which uses a cold cathode field emission as an x-ray source. To increase the contact of our semiconductor samples with metal to prevent the charging due to the accumulation of positive charge on the sample and using the sample for multiple measurements, we designed Ti crucibles. The powder samples are contained inside the crucible, as you can see in the optical image in Figure 2.10.
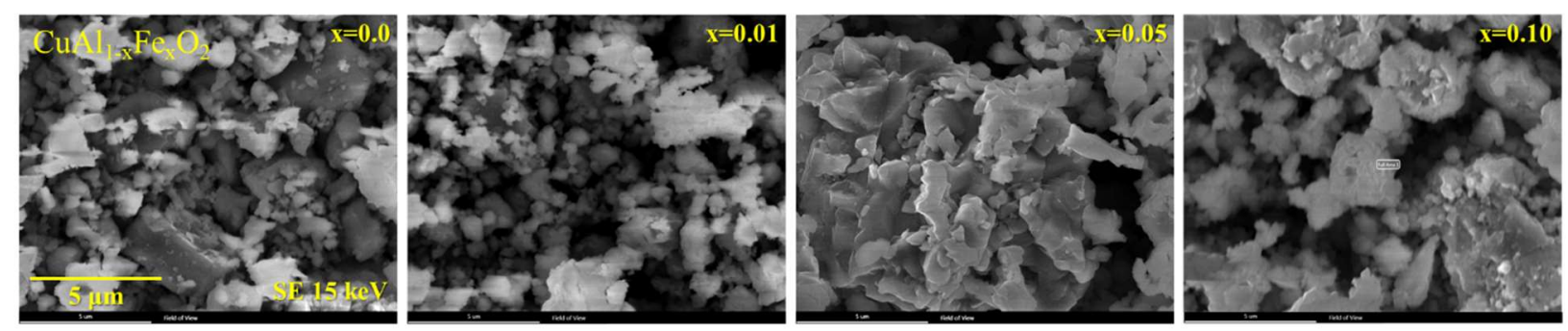

Figure 2.11: shows four secondary electron images of the powder samples taken at $9 \mathrm{k}$ magnification, $15 \mathrm{kV}$ acceleration voltage, and $12 \mathrm{~cm}$ working distance. 

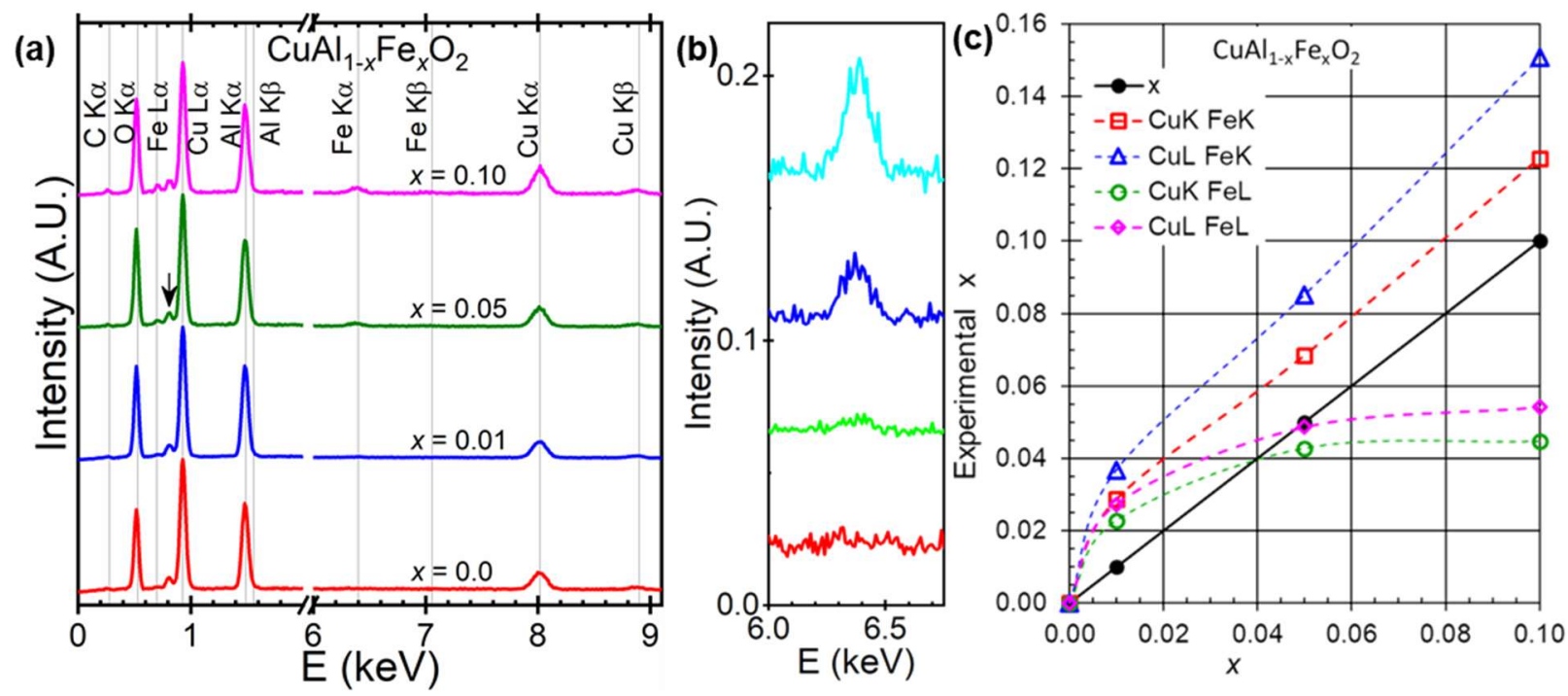

Figure 2.12: (a) shows the EDS spectra for $\mathrm{CuAl}_{1-x} \mathrm{Fe}_{x} \mathrm{O}_{2}$ samples. The energy edges of each element are marked by grey lines. The arrow shows the artifact sum peak due to $\mathrm{C} \mathrm{K} \alpha$ and $\mathrm{O} K \alpha$ peaks. (b) is the blow up of Fe $\mathrm{K} \alpha$ monotonous increase vs. iron concentration. (c) shows the measured iron concentration using various ZAF corrections associated with different combination of the energy edges. The black solid line shows the expected concentration.

Secondary electron microscopy (SEM) and energy dispersive (x-ray) spectroscopy (EDS) was performed on $\mathrm{CuAl}_{1-x} \mathrm{Fe}_{x} \mathrm{O}_{2}$ for $\mathrm{x}=0.0,0.01,0.05$, and 0.10 samples. An SEM image and EDS spectrum are shown for all four samples (see Figures 2.10 and 2.11, respectively). The SEM image shows a powder with a distribution of particle sizes, consistent with earlier Scherrer analysis [7]. The image is taken at 15 $\mathrm{kV}$ accelerating voltage, $10 \mu \mathrm{A}$ electron beam current, and $12 \mathrm{~cm}$ working distance. The EDS signal is collected from the whole region shown in Figure 2.11. The EDS spectra for all four samples are shown in Figure 2.12 (a). the $\mathrm{x}$-axis between 2 and 6 is broken as there was no peak in that range. From the usual elemental quantification (ZAF), the concentration of $\mathrm{C}$ was about $15 \%$ for all samples, which is typical for an air-exposed powder. Figure 2.12 (b) shows the Fe Kal peak for $x=0.0,0.01,0.05$ and 0.10 . This Fe peak monotonically increases with nominal $x$ value. Figure 2.12 (c) is a plot showing EDS determined $x$ values vs. nominal $x$ values using several calculation methods. These methods include ZAF analysis using the $\mathrm{Cu}$ and $\mathrm{Fe} \mathrm{K}$ lines (open squares); ZAF using $\mathrm{Cu}$ and $\mathrm{Fe} \mathrm{L}$ lines (open diamonds), and the ratio between the $\mathrm{Fe}$ and $\mathrm{Cu} \mathrm{K}$ lines (corrected for the fluorescence differences between $\mathrm{Fe}$ and $\mathrm{Cu}$ ) (open triangles), (dashed lines are visual guides, and the solid black line shows the expected value of $x$ vs. nominal). The Fe to $\mathrm{Cu}$ ratio shows the expected linear relationship, with exaggerated measured $x$, which is probably the 
result of the $\mathrm{Z}$ dependence in the EDS process not considered. The K-line ZAF analysis shows the best quantitative agreement, with a small systematic exaggeration of the measured $x$. The L-line ZAF analysis poor agreement with saturated behavior. This poor agreement is probably because the Fe L $\alpha$ peak is a small peak on the shoulder of the $\mathrm{Cu} \mathrm{L} \alpha$ peak, whereas the $\mathrm{Fe}$ and $\mathrm{Cu} \mathrm{K}$ peaks a well separated. Using the $\mathrm{K}$-line ZAF analysis, disregarding the carbon, the elemental ratios are given by $\mathrm{Cu}_{1.00} \mathrm{Al}_{0.68} \mathrm{Fe}_{0.09} \mathrm{O}_{1.20}$, as compared with the nominal $\mathrm{Cu}_{1} \mathrm{Al}_{0.90} \mathrm{Fe}_{0.10} \mathrm{O}_{2}$. The measured elemental ratios are within the expected range for EDS on powders without calibration with standards. Certainly, the EDS measurements support the nominal elemental Fe content for these samples. The peak that is marked by the arrow in Figure 2.12 (a) is present in all of the samples and cannot be a peak from any of the elements. These peaks might be an artifact peak called Sum peak $(0.91 \mathrm{eV})$ arise from detector error in recording the $\mathrm{C} \mathrm{K} \alpha(0.28 \mathrm{eV})$ and $\mathrm{O} \mathrm{K} \alpha(0.53 \mathrm{eV})$ energies as an individual peak [8]. 


\subsection{Chemical Analysis by X-ray Photoelectron Spectroscopy (XPS):}

\subsubsection{XPS Overview:}

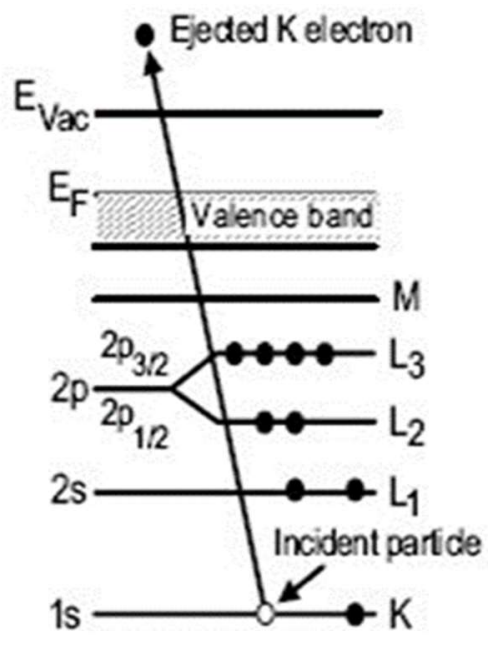

(a) Ionization

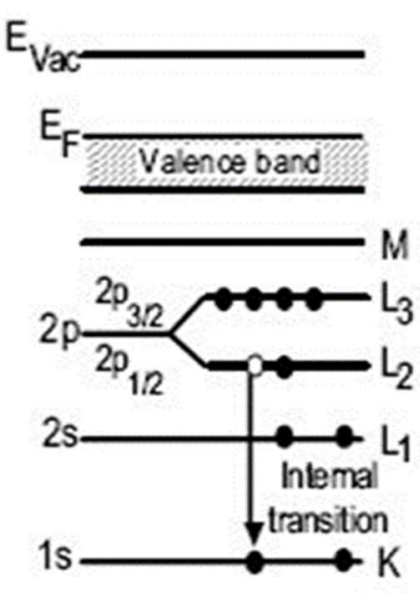

(b) Relaxation

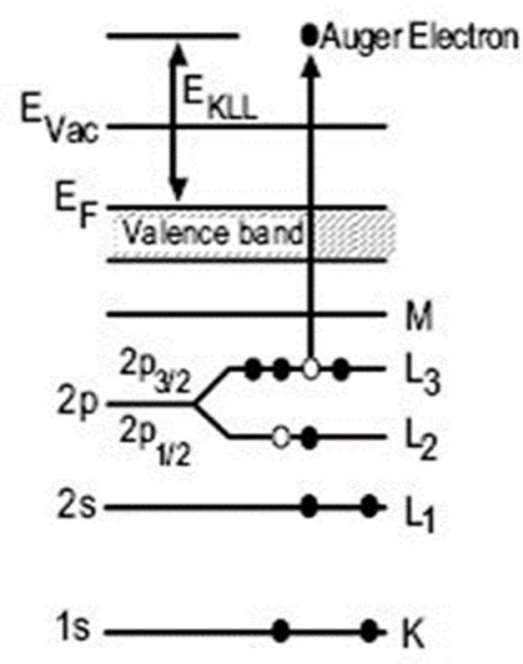

(c) Emission

Figure 2.13: (a) shows when x-ray interact with the atoms of the sample bound electron will be ejected with specific quantized energies which is the photoelectric effect (ionization process), (b) and (c) shows electrons form higher energy levels will replace the lower energy level (relaxation process) and emit x-ray energy which can cause the electron with similar binding energy to be ejected (auger electrons).

X-ray photoelectron spectroscopy (XPS) is a well-established surface analysis technique capable of providing elemental and chemical state information from the outer 5 to $10 \mathrm{~nm}$ of a solid surface. Hence, this is not an appropriate technique for the bulk sample, and for the estimation of elemental composition, EDS is more suitable. When an $\mathrm{x}$-ray of known energy (hv), generated from either $\mathrm{Al} \mathrm{K} \alpha$ at $1486.7 \mathrm{eV}$ or $\mathrm{Mg} \mathrm{K} \alpha$ at $1253.6 \mathrm{eV}$, interacts with an atom, because of the photoelectric effect, a photoelectron can be emitted. The photoelectron process is shown in Figure 2.13 (a). Note that the selection of the x-ray source does not affect the binding energy of the main peaks. However, the Auger peaks shift depending on the selection of the x-ray source wavelength. The emitted electron's kinetic energy $\left(E_{k}\right)$ can be measured, and the atomic core level binding energy $\left(\mathrm{E}_{\mathrm{b}}\right)$ relative to the Fermi level $\left(\mathrm{E}_{\mathrm{F}}\right)$ of the sample can be determined using the following equation [68]:

$E_{b}=h v-E_{k}-\phi_{s p}$ 
where $\Phi_{\mathrm{sp}}$ is the work function of the spectrometer. To identify the concentration of the elements within $10 \mathrm{~nm}$ of the surface, various core level binding energies observed in a spectrum can be used except for hydrogen and helium. Thanks to the binding energies sensitivity to the chemical environment of the atom, the chemical state information can also be extracted, which means the binding energy of the peaks can change, and there can be more than one peak observed. By deconvoluting the peaks and comparing the binding energies with reported computational and experimental values, the type of bonding and the chemical environment will be determined. Overall, binding energies increase when the atom of interest participates in strong bonds, whereas, decrease when core electrons of atoms withdraw electrons from their neighboring atoms [68].

A thin layer of 1-2 $\mathrm{nm}$ carbonaceous material is usually found on the surface of air-exposed samples. This layer is generally known as adventitious carbon. Even small exposures to the atmosphere can produce these films. Adventitious carbon is mostly comprised of a variety of hydrocarbons species with small amounts of both singly and doubly bound oxygen functionality, containing C-C, C-H, C-O-C, and O$\mathrm{C}=\mathrm{O}$ chemical bonds. The source of this carbon is ambiguous. It cannot be graphitic in nature, and in most modern high vacuum systems, vacuum oils are not readily present. It is possible that it is produced by CO or $\mathrm{CO}_{2}$ species [69].

The deconvoluted spectra will give us the percentage of each species as a function of each element. The actual amount of oxygen needs to be estimated to determine the concentration of each chemical state of elements. The oxygen amount associated with the adventitious Carbone can be determined from the detailed $\mathrm{C} 1 \mathrm{~s}$ peak scan and subtract it from the detailed scan of $\mathrm{O} 1 \mathrm{~s}$ and finally consider the exact amount of oxygen in the survey scan. The adventitious carbon $\mathrm{C} 1 \mathrm{~s}$ from the $\mathrm{C}-\mathrm{C}$ component $(284.8 \mathrm{eV})$ is commonly used as a charge reference for XPS spectra. 


\subsubsection{Delafossite Alloys Chemical Analysis:}

The chemical characterization of the samples was performed using a PHI 5000 VersaProbe x-ray photoelectron spectroscopy system with an $\mathrm{Mg}$ conventional x-ray source with a $5 \mathrm{~mm}$ beam cross-section. The $\mathrm{Al}$ focused $\mathrm{x}$-ray source is not used as the Fe $2 \mathrm{p}$ peaks would overlap with $\mathrm{Cu}$ Auger peaks, and for small Fe, concentration made the estimation of the concentration hard. The same titanium crucibles are used for the measurement.

$\mathrm{X}$-ray photoelectron spectroscopy (XPS) is done on all $\mathrm{CuAl}_{1-x} \mathrm{Fe}_{x} \mathrm{O}_{2}$ powder samples. As all the sample XPS spectra are similar, the spectra and the deconvolution of the peaks for $x=0.10$ samples are showing. Figure 2.14 (a) shows the survey scan (step size $0.5 \mathrm{eV}$ ) for $\mathrm{CuAl}_{0.9} \mathrm{Fe}_{0.1} \mathrm{O}_{2}$. Figure 2.14 (b-f) is the detailed scans (step size $0.1 \mathrm{eV}$ ) of the strongest intensity peaks. The background is determined by the Shirley method. The peaks are deconvoluted by functions that are a mixture of Lorentzian and Gaussian functions. Details of the peak fitting are also indicated for all the spectra. The C 1s peaks $(\sim 285 \mathrm{eV})$ are the adventitious carbon highest intensity peak. It is fitted to an alkane $(\mathrm{C}-\mathrm{C}, \mathrm{C}-\mathrm{H})$, alcohol $(\mathrm{C}-\mathrm{OH})$, and acid/ester $(\mathrm{O}-\mathrm{C}=\mathrm{O})$ peaks (See Figure $2.14(\mathrm{c}))$. The oxygen associated with the adventitious carbon should be estimated based on the $\mathrm{C}$ 1s detailed scan and be subtracted from the total oxygen peak percentage in the survey scan. The $\mathrm{O} 1 \mathrm{~s}$ peak $(\sim 530 \mathrm{eV})$ is fitted with three lattice peaks associated with $\mathrm{Al}^{3+}, \mathrm{Cu}^{+}, \mathrm{Cu}^{2+}$, along with organic and water peaks, shown in Figure 2.14 (d). Figure 2.14 (f) shows $\mathrm{Cu} 2 \mathrm{p}$ peaks ( 935 eV) which is fitted as $\mathrm{Cu}^{1+}$ and $\mathrm{Cu}^{2+} 2 \mathrm{p}$ peaks. These two contributions are easily distinguishable as the 

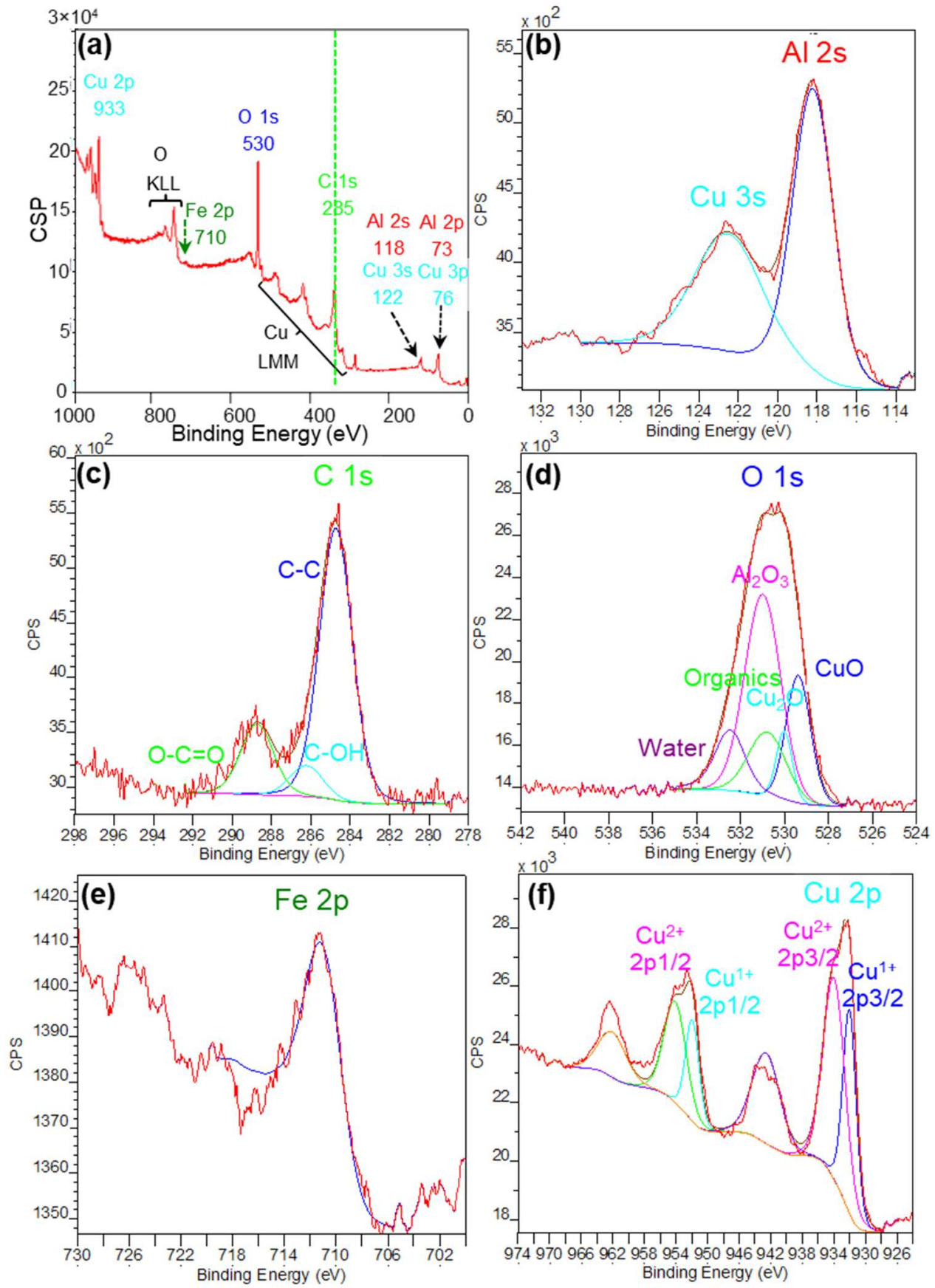

Figure 2.14: (a) XPS survey scan for $x=0.10$ using a conventional Mg x-ray source, (b-f) are the detailed scans for labeled peaks, respectively, $\mathrm{Al} 2 \mathrm{~s}$, adventitious $\mathrm{C} 1 \mathrm{~s}, \mathrm{O} 1 \mathrm{~s}, \mathrm{Fe} 2 \mathrm{p}$, and $\mathrm{Cu} 2 \mathrm{p}$. expanded as indicated. Chemical species in the detailed scans (inset) are labeled in the same color as the curve used to fit the data. Upper right insert is a table of peak height ratios.

$\mathrm{Cu}^{2+}$ has two satellite peaks related to $\mathrm{Cu}^{2+} 2 \mathrm{p} 3 / 2$ and $\mathrm{Cu}^{2+} 2 \mathrm{p} 1 / 2$. Fe $2 \mathrm{p}$ peak $(\sim 711 \mathrm{eV})$ is fitted as $\mathrm{Fe}^{3+}$. The $\mathrm{Cu} 3 \mathrm{~s} / \mathrm{Al} 2 \mathrm{~s}$ combined peaks $(\sim 120 \mathrm{eV})$ are fitted as separate $\mathrm{Cu} 3 \mathrm{~s}$ Al 2 s peaks (where the area of $\mathrm{Cu}$ $3 \mathrm{~s}$ is consistent with that from $\mathrm{Cu} 2 \mathrm{p}$ peaks) in Figure 2.13 (b). The alkane $\mathrm{C}$ peak can be disregarded to 
determine the chemical makeup of our powder but use the alcohol and acid/ester related peaks to determine their contribution to the Oxygen peak. For the Oxygen content, the contribution of the organic $\mathrm{O}$ as well as the $\mathrm{O}$ in water is disregarded. The size of the lattice peaks for $\mathrm{Al}^{3+}, \mathrm{Cu}^{1+}$, and $\mathrm{Cu}^{2+}$ are consistent in the area from the associated $\mathrm{Al} 2 \mathrm{~s}$ and $\mathrm{Cu} 2 \mathrm{p}$ lines. Thus, the procedure is designed to determine the $\mathrm{Cu}^{1+}, \mathrm{Cu}^{2+}, \mathrm{Al}$, and $\mathrm{O}$ (lattice only) content for the powders. Table 2.1 contains the ratios for $\frac{\mathbf{F e} 2 p_{3 / 2}}{\mathbf{A l ~} 2 s}$ and $\frac{\mathbf{C u}^{1+}}{\mathbf{C u}^{1+}+\mathbf{C u}^{2+}}$. The former is related to the experimentally determined $x$. The table shows this ratio monotonically increases with the nominal value of $x$, as expected. However, the ratio is much less than $x$ and appears to saturate. The latter ratio measures the amount of the different copper valences of $1+$ and $2+$. This ratio indicates that within the surface layer probed by XPS (about $10 \mathrm{~nm}$ ), there is a near-even mixture of $\mathrm{Cu}^{1+}$ and $\mathrm{Cu}^{2+}$ with a systematic trend to lower $\mathrm{Cu}^{1+}$ for higher $\mathrm{Fe}$ content. The delafossite has $\mathrm{Cu}^{1+}\left(\right.$ spinel $\left.\mathrm{Cu}^{2+}\right)$, and it is not surprising that at the surface, there is further oxidation leading to more $\mathrm{Cu}^{2+}$. This may be where the small amount of the spinel phase, as measured using $\mathrm{XRD}$, tends to be located. Moreover, this $\mathrm{Cu}^{2+}$ may also contribute to the paramagnetism described in the magnetic properties chapter [8].

Table 2.1: shows the ratio of the $\mathrm{Fe}$ to $\mathrm{Al}$ strongest peaks and the $\mathrm{Cu}^{1+}$ to the total $\mathrm{Cu}$.

\begin{tabular}{|c|c|c|c|c|}
\hline Nominal $\boldsymbol{x}$ & $\mathbf{0 . 0 0}$ & $\mathbf{0 . 0 1}$ & $\mathbf{0 . 0 5}$ & $\mathbf{0 . 1 0}$ \\
\hline$\frac{\mathbf{F e} 2 \boldsymbol{p}_{\mathbf{3} / \mathbf{2}}}{\mathbf{A l ~ 2 s}}$ & 0.00 & 0.005 & 0.014 & 0.019 \\
\hline$\frac{\mathbf{C u}^{\mathbf{1 +}}}{\mathbf{C u}^{\mathbf{1 +}}+\mathbf{C u}^{\mathbf{2 +}}}$ & 0.44 & 0.38 & 0.37 & 0.28 \\
\hline
\end{tabular}

\subsection{Summary and Conclusion:}

We synthesized $\mathrm{CuAl}_{1-x} \mathrm{Fe}_{x} \mathrm{O}_{2}$ alloys by solid-state reaction of the oxide precursors by optimizing the synthesis parameters. Our XRD measurements confirmed the substitution of Fe in Al sites and the rhombohedral crystal structure. By Rietveld refinement, the phases in the powders are quantified. The elemental characterization of the samples was performed, which confirmed the monotonically increasing Fe contentment vs. nominal $x$. From the XPS results, a significant amount of $\mathrm{Cu}^{2+}$ on the surface of the 
particles is observed. $\mathrm{Cu}^{2+}$ can generate a paramagnetic background in the magnetic measurements. Moreover, it can lead to the $\mathrm{Cu}^{2+} / \mathrm{Cu}^{+}$transition absorption feature in the optical UV-Vis measurements. 


\section{Chapter 3}

\section{Computational Study of Dilute Delafossite alloys}

\subsection{Overview:}

In this chapter, I discuss the details of the computational study using density functional theory (DFT) to model the phonon, electrical, and optical dynamics for the $\mathrm{CuAl}_{1-\mathrm{x}} \mathrm{Fe}_{\mathrm{x}} \mathrm{O}_{2}$. This is how the phonon dispersion and the electronic band structure for a supercell are calculated. In particular, I introduce a weighted dynamical matrix (WDM) approach that overcomes the difficulties involving the intensive calculations usually required to model the phonons dispersion for the dilute magnetic alloys. The data presented here are compared to the experimental results in Chapters 5 and 6.

\subsection{Very Short Overview of Density Functional Theory:}

Any crystal structure can be described as a system of electrons and nuclei that interact with each other based on quantum mechanics. The ground-state of the energy of this system can be calculated by solving the corresponding many-body Schrödinger equation, $H \Psi=E \Psi$, where $H$ is the Hamiltonian operator, $\Psi$ is the many-body wave function, and $E$ is the total energy. The Hamiltonian for a system of interacting electrons and nuclei can be written as follows:

$H(\boldsymbol{R}, \boldsymbol{r})=T_{N}(\boldsymbol{R})+T_{e}(\boldsymbol{r})+V_{N N}(\boldsymbol{R})+V_{e e}(\boldsymbol{r})+V_{e N}(\boldsymbol{R}, \boldsymbol{r})$

where $\boldsymbol{R}$ are the nuclei coordinates, and $\boldsymbol{r}$ is the electrons coordinates, $T_{N}$ and $T_{e}$ are the kinetic energy operators for the nuclei and electrons, respectively, and $V_{N N}, V_{e e}$, and $V_{e N}$ are the electrostatic potential energy operators among nuclei, electrons, and electrons, and nuclei, respectively. The solution of this equation is computationally demanding and is only tractable for simple systems. Therefore, some approximations are crucial to study the properties of complex systems. Below we outline these approximations.

The first approximation is the Born-Oppenheimer approximation [70] that takes advantage of the nuclei masses being much larger than electrons mass $\left(M_{N} \gg m_{e}\right)$. Hence, the kinetic energy of nuclei is 
not significant compared to that of the moving electrons and can be neglected ( $\left.T_{N} \cong 0\right)$. This assumption simplifies the Hamiltonian by decoupling the electronic from the nuclear of the wave function. The interactions between electrons and nuclei can be approximated by an external potential $V_{\text {ext }}(\boldsymbol{r})$ and the Hamiltonian simplifies to:

$H_{e}(\boldsymbol{R}, \boldsymbol{r})=T_{e}(\boldsymbol{r})+V_{N N}(\boldsymbol{R})+V_{e e}(\boldsymbol{r})+V_{e x t}(\boldsymbol{r})$.

Even though the Born-Oppenheimer approximation is applied, still solving the above equation for interacting electrons of $N$ ions in a macroscopic solid $\left(N \sim 10^{23}\right)$ is intractable because of the complexity of the many-body wave function. In practice, further simplifications and approximations are necessary to estimate the electron-electron interactions. One very successful approach is density functional theory (DFT), which was formulated by Hohenberg, Kohn [71], and Sham [72] to estimate the many-body electron-electron interactions. Hohenberg and Kohn built the basis of DFT based on two theorems:

Theorem 1: The external potential $V_{\text {ext }}(\boldsymbol{r})$ exerted on a many-body system is uniquely determined by the ground-state electron density $n_{0}(\boldsymbol{r})$ of that system.

Theorem 2: For any given external potential $V_{\text {ext }}(\boldsymbol{r})$, there exists a universal functional $E_{H K}[n(\boldsymbol{r})]$ for energy in terms of electron density $n(\boldsymbol{r})$. The global minimum of this functional is the ground state energy, and the density that minimizes the functional is the ground-state electron density $n_{0}(\boldsymbol{r})$.

The important improvement of DFT for the calculation of the energy of a many-body system is to use the electron density as the only variable, instead of the electronic wave function. For an N-electron system, this greatly reduces the number of degrees of freedom from $3 N$ to 3 (the $x, y, z$ spatial coordinates of the electron density). The energy can be expressed as a function of the electron density: $E=E(n)$, and the electron density $n$ is also a function of the electron coordinates $r(n=n(r))$. Hence, the energy is a functional of the electron density $(E=E[n(r)])$. (A functional means a function of another function and is indicated by the square brackets.) The total energy under the Hohenberg and Kohn theorem can be rewritten as the following:

$E_{H K}[n(\boldsymbol{r})]=T[n(\boldsymbol{r})]+E_{e e}[n(\boldsymbol{r})]+E_{\text {ext }}[n(\boldsymbol{r})]+E_{N N}$, 
where $T$ represents the kinetic energy of the electrons and $E_{e e}$ represents the electron-electron interaction. $E_{\text {ext }}=\int V_{\text {ext }}(\boldsymbol{r}) n(\boldsymbol{r}) d \boldsymbol{r}$ is the interaction between the external potential and the electrons, which can be the potential from the nuclei $E_{e N}$, or an external field such as an electric field or a magnetic field, and $E_{N N}$ is the interaction between the nuclei.

The most common implementation of DFT is through the Kohn-Sham approach that maps the system of many interacting electrons onto a system of non-interacting particles leading to the same groundstate density as the real system. This mapping makes the problem tractable and the quantum mechanical effects that are not included in the "known" terms are included in an exchange-correlation functional of the density. In this framework, the density through the one-electron orbital $\psi_{i}(\boldsymbol{r})$ and the total electronic energy is given as follows:

$$
\begin{aligned}
& n(\boldsymbol{r})=\sum_{i}\left|\psi_{i}(\boldsymbol{r})\right|^{2}, \\
& E_{K S}[n(\boldsymbol{r})]=E_{\text {known }}[n(\boldsymbol{r})]+E_{x c}[n(\boldsymbol{r})], \\
& E_{K S}[n(\boldsymbol{r})]=\left\langle\psi_{i}\left|T[n(\boldsymbol{r})]+V_{e e}[n(\boldsymbol{r})]+V_{e x t}[n(\boldsymbol{r})]+V_{N N}+V_{x c}[n(\boldsymbol{r})]\right| \psi_{i}\right\rangle, \text { and } \\
& E_{K S}[n(\boldsymbol{r})]=\left\{-\frac{1}{2} \sum_{i}\left\langle\psi_{i}\left|\nabla^{2}\right| \psi_{i}\right\rangle+\frac{1}{2} \int \frac{n(\boldsymbol{r}) n\left(\boldsymbol{r}^{\prime}\right)}{\left\|\boldsymbol{r}-\boldsymbol{r}^{\prime}\right\|} d \boldsymbol{r} d \boldsymbol{r}^{\prime}+\int V_{e x t}(\boldsymbol{r}) n(\boldsymbol{r}) d \boldsymbol{r}+E_{N N}\right\}+E_{x c}[n(\boldsymbol{r})] .
\end{aligned}
$$

In Equation(3.7), the $1^{\text {st }}$ term corresponds to the electron kinetic energy; the $2^{\text {nd }}$ term is Coulomb interactions between pairs of electrons (the Hartree energy, $E_{H}$ ) the $3^{\text {rd }}$ term is the energy of electrons in the external potential $V_{\text {ext }}$ due to the nuclei, the $4^{\text {th }}$ term is the interaction between nuclei, and the $5^{\text {th }}$ and last term is the exchange and correlation energy between electrons. All of these terms are known except the exchange-correlation energy functional $E_{x c}[n(\boldsymbol{r})]$. The Hartree energy $\left(2^{\text {nd }}\right.$ term $)$ includes a so-called selfinteraction that involves the Coulomb interaction between the electron and itself. The self-interaction is not physical and needs to be corrected along with several other effects that are gathered into the final energy in the Kohn-Sham equations, $E_{x c}$, which involves the exchange and correlation between electrons. If the functional $E_{x c}[n(\boldsymbol{r})]$ is provided, then the ground-state energy and density of the problem of the many- 
body electron can be determined. For a given set of atomic positions, the ground state is determined via the minimization of $E_{K S}$ giving rise to the total energy and the electronic density of the system.

Unfortunately, the exact exchange-correlation term is not known, and approximations are needed to estimate this term. The most common approximation is to consider the electron density constant at all points in space [73], that is, $n(\boldsymbol{r})=$ constant. This approximation was proposed by Kohn and Sham, and is called the local density approximation (LDA) [72] where the exchange-correlation energy per electron is only depend on the density at this point, and where the exchange-correlation energy of a non-uniform electron system is approximated by that of a uniform electron gas with that density, so that

$E_{x c}^{L D A}[n]=\int \epsilon_{x c}[n(\boldsymbol{r})] n(\boldsymbol{r}) d \boldsymbol{r}$.

The exchange part of the homogeneous gas can be obtained from using the Hartree-Fock technique, which gives a simple analytic form:

$\epsilon_{x c}[n(\boldsymbol{r})]=-\frac{3}{4 \pi}\left(3 \pi^{2} n(\boldsymbol{r})\right)^{1 / 3}$

LDA is most valid in the uniform density limit. Even though it appears too simple, it works remarkably well in many cases, which makes this approximation the most widely used in solid-state simulations. Typical errors associated with LDA in comparison to experimental measurements are about $1 \%$ on atomic positions and lattice constants and $5 \%$ on phonon frequencies. The largest error is in the electronic bandgap, which is underestimated compared to experimental values.

Another common class of functionals uses information about the local electron density and the local gradient in the electron density. This generalized gradient approximation (GGA) [74] accepts that, in reality, electron densities are not uniform and include this spatial variation in the electron density that can make more inclusive functionals to describe a wider range of materials. Note that the equations on which the GGA are based are valid for slowly varying densities. In the GGA, the exchange-correlation functional includes the local electron density and gradient in the electron density: 
$E_{x c}^{G G A}[n(\boldsymbol{r})]=\int \epsilon_{x c}[n(\boldsymbol{r}),|\nabla n(\boldsymbol{r})|] n(\boldsymbol{r}) d \boldsymbol{r}$.

Even though GGA functionals include many terms, it is often thought that non-empirical GGA functionals should result in more accurate predictions than the LDA functionals. This is quite often the case, but there are exceptions. One exception is in the calculation of the surface energy of transition metals and oxides. Because there are many ways in which information from the gradient of the electron density can be included in a GGA functional, there are many ways GGA functionals can be developed and used. In our calculations, we use the Perdew-Burke-Ernzerhof functional (PBE) [75], which is one of the most wellknown functionals.

In general, when compared to LDA, GGA gives a better description of energetic properties and is more applicable to chemical modeling, but it also overestimates the lattice constants and bond lengths. In addition, just like LDA, GGA fails to describe strongly correlated systems, such as transition metal oxides, where the $d$-electrons are strongly correlated with each other via the Coulomb interaction. A famous example is that DFT predicts the $\mathrm{CoO}$ to be metallic, while it is actually an antiferromagnetic insulator.

To accurately calculate the bandgap values of strongly correlated systems, DFT $+U[76]$ has been developed, where $U$ is the Hubbard correction taking into account. The total energy in the GGA+U method is:

$E_{G G A+U}=E_{G G A}[n(r)]+E_{H u b}\left[\left\{n^{\sigma}\right\}\right]-E_{d c}\left[\left\{n^{\sigma}\right\}\right]$

where $\sigma$ shows the spin index, $n(r)$ is the electron density and $\left\{n^{\sigma}\right\}$ is the density matrix of $\mathrm{d}$ or $\mathrm{f}$ electrons for spin $\sigma .1^{\text {st }}$ term is the standard GGA energy functional with spin, $2^{\text {nd }}$ term $E_{H u b}$ is the Hubbard term that contains electron-electron Coulomb interactions, and the last term $E_{d c}$ is a so-called double-counting term that eliminates the part of the interaction energy already contained in $E_{H u b}$ to avoid double-counting problems. The matrix form of Equation (3.11) can be written as:

$E_{G G A+U}=E_{G G A}+\frac{1}{2} U \sum_{m, \sigma \neq m^{\prime}, \sigma^{\prime}} n_{m}^{\sigma} n_{m^{\prime}}^{\sigma^{\prime}}-\frac{1}{2} U N(N-1)$ 
where $m$ denotes the magnetic quantum number and $N=\operatorname{Tr}\left(n_{m m^{\prime}}^{\sigma}\right)$. The orbital energies $\varepsilon_{i}$ is:

$\varepsilon_{i}=\frac{\partial E}{\partial n_{i}}=\varepsilon_{G G A}+U\left(\frac{1}{2}-n_{i}\right)$

Where $n_{i}$ denotes the orbital occupations. The dependency on the occupation number is expected as the Hubbard correction is only applied to the states that are most disturbed by correlation effects. In this simple consideration, the GGA orbital energies are shifted by $-U / 2$ for occupied orbitals $\left(n_{i}=1\right)$ and by $+U / 2$ for unoccupied orbitals $\left(n_{i}=0\right)$, resulting in lower and upper Hubbard bands separated by $\mathrm{U}$, which opens a gap at the Fermi energy in transition metal oxides. However, the bandgap is not completely corrected since it still suffers from underestimation due to other DFT-inherent causes such as selfinteraction errors. We note that adjusting $U$ to achieve the experimental band gap or adding $U$ terms on states other than the highly localized semi-core states is unjustified. Another complication is the choice of the $U$ parameter.

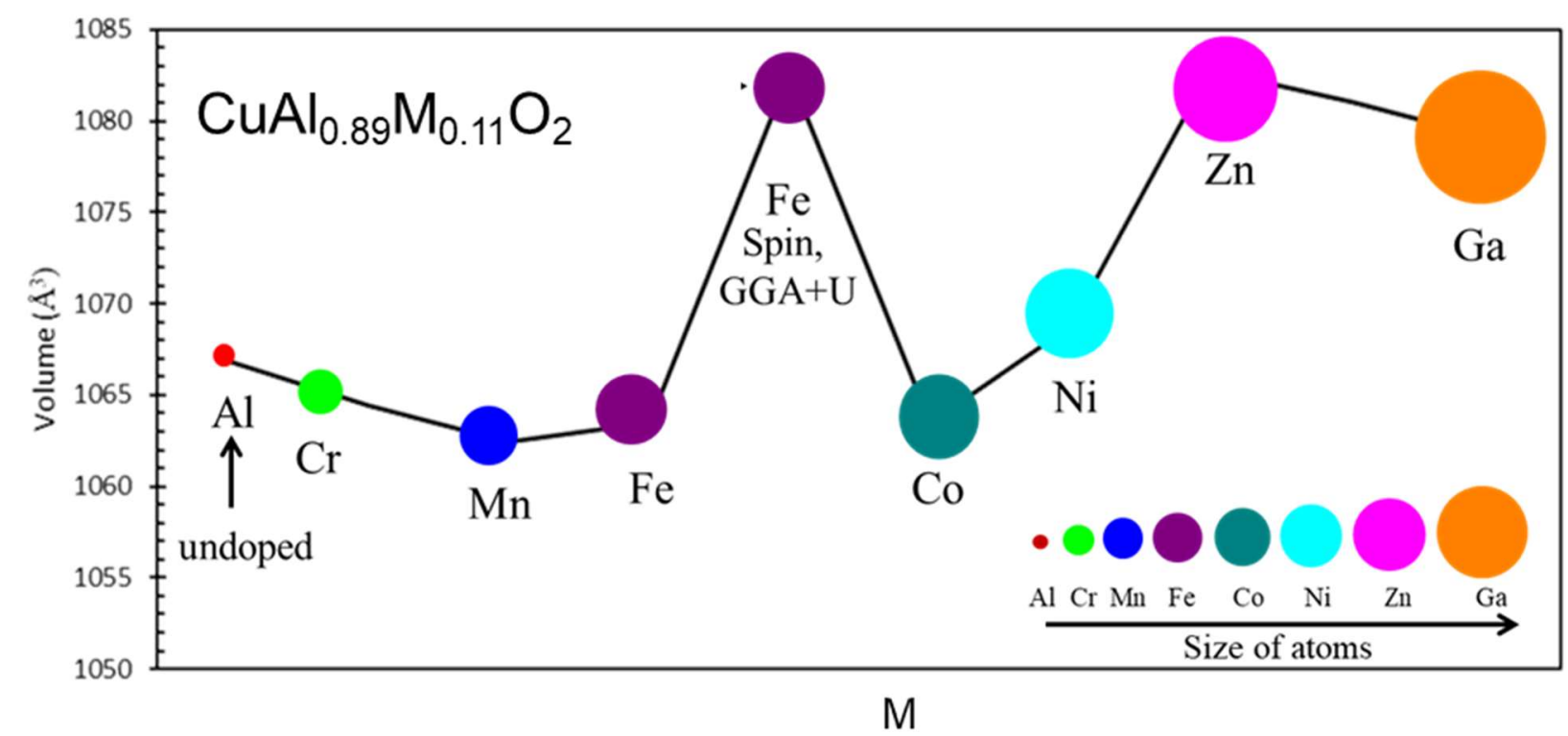

Figure 3.1.: Volume of conventional unit cell is calculated for $3 \times 3 \times 1$ supercells of $\mathrm{CuAl}_{0.89} \mathrm{M}_{0.11} \mathrm{O}_{2}(\mathrm{M}=\mathrm{Cr}, \mathrm{Mn}$, $\mathrm{Fe}, \mathrm{Co}, \mathrm{Ni}, \mathrm{Zn}$, and $\mathrm{Ga}$ ) using spin polarized GGA for all the alloys. For M = Fe GGA+U showsbetter agreement with the experimental values and the Vegrad's law prediction.

To illustrate the appropriateness of DFT $+U$ calculation in our study, we calculated the conventional unit cell volume by supercells of $3 \times 3 \times 1$ (108 atoms) using spin-polarized (GGA $+U$ ) in VASP (Vienna Ab 
initio simulation package) package for $\mathrm{CuAl}_{0.89} \mathrm{M}_{0.11} \mathrm{O}_{2}(\mathrm{M}=\mathrm{Cr}, \mathrm{Mn}, \mathrm{Fe}, \mathrm{Co}, \mathrm{Ni}, \mathrm{Zn}$, or Ga). Figure 3.1 illustrates the volume for each alloy. Because all $\mathrm{M}$ elements are larger than $\mathrm{Al}$, we expect an increase in the lattice parameters and the unit cell volume. Only for $\mathrm{M}=\mathrm{Fe}$ the Hubbard correction was considered. Our test showed that just considering the spin does not improve the lattice parameters sufficiently from this. We conclude that for transition metals, it is necessary to consider the Hubbard correction $U$ to calculate more accurate lattice parameters.

Other functionals have been developed to deal with other underestimations in the DFT calculation. However, these are beyond the scope of discussion in this thesis.

\subsection{Calculation of Lattice Parameters:}

Our computational strategy is the following: we use the supercell approach to determine the lattice parameters of the $\mathrm{CuAl}_{1-x} \mathrm{Fe}_{x} \mathrm{O}_{2}$ alloys for $x=0,0.01,0.05,0.1$, and 1.0 using Vienna $\mathrm{Ab}$ initio Simulation Package (VASP 5.4.4), see Figure 2.7 (b) and (c).

The lattice parameters for $\mathrm{CuAl}_{1-x} \mathrm{Fe}_{x} \mathrm{O}_{2}$ are calculated in its ground-state energy configuration using VASP [77-79]. The relaxation of the conventional unit cell is done with an $8 \times 8 \times 2 \Gamma$-centered $k$-point mesh and kinetic energy cut-off of $600 \mathrm{eV}$. The rhombohedral conventional unit cell $(R \overline{3} m)$ for $\mathrm{CuAlO}_{2}$ is used to construct a $3 \times 3 \times 1$ supercell containing 108 atoms. We use projector-augmented wave pseudopotentials [80] with the valence-electron configurations $3 p^{6} 3 d^{10} 4 s^{1}, 3 p^{6} 3 d^{7} 4 s^{1}, 3 s^{2} 3 p^{1}$, and $2 s^{2} 2 p^{4}$ for $\mathrm{Cu}, \mathrm{Fe}, \mathrm{Al}$ and $\mathrm{O}$, respectively. There are many possible configurations for Fe substituting for Al, but most of them can be ruled out by symmetry considerations, which is done by a site-occupation disorder (SOD 0.47 ) code [81]. The relaxation of the disordered supercell is done with a $4 \times 4 \times 2 \Gamma$-centered $k$-point mesh and $600 \mathrm{eV}$ kinetic energy cut-off. The relaxation method used leaves the supercell volume, positions, and cell shape free to change. Due to the strong correlation effects of Fe $d$ electrons, we used a simplified (rotationally invariant) $\mathrm{PBEsol}+U$ approach $[82,83]$ with on-site Coulomb parameter $U=4.6 \mathrm{eV}$ and 
include spin polarization for Fe. The bandgap, lattice parameters, magnetic moment, and Raman modes of $\mathrm{CuFeO}_{2}$ are compared with the reported experimental values to find the optimized $U$.

The observed percentage changes in the lattice parameters $a, c$, and volume $V$ with an increase in $x$ determined from the XRD patterns are plotted in Figure 2.7 (a). The \% changes in $a$ are larger than those in $c$, confirming the substitution of the larger $\mathrm{Fe}^{3+}$ for $\mathrm{Al}^{3+}$ because the $\mathrm{Al}$ sites are in the layers normal to the $c$ direction and are bonded to oxygens, see Figure 2.7 (b). The solid black lines in Figure 2.7 (a) show the fit to Vegard's equation using $a_{\mathrm{CuAlO}_{2}}=2.86 \AA$ and $a_{\mathrm{CuFeO}_{2}}=3.04 \AA$, and $c_{\mathrm{CuAlO}_{2}}=16.96 \AA$, and $c_{\mathrm{CuFeO}_{2}}=17.17 \AA$. The samples have rhombohedral delafossite structure, and there is a lattice expansion associated with replacing $\mathrm{Al}$ with $\mathrm{Fe}$, which shows up as the peaks shift to lower angles $[7,48]$. A more detailed explanation of the lattice parameter dynamics is in Chapter 2.

\subsection{Challenges of Phonon Calculation:}

In this section, $\mathrm{I}$ describe the calculation of vibrational modes for dilute $\mathrm{CuAl}_{1-x} \mathrm{Fe}_{x} \mathrm{O}_{2}$ alloys from first principles. Specifically, I am interested in the behavior of these vibrational modes with Fe content and their comparison to the experimental results in Sect. 5. The calculation of vibrational frequencies (phonon spectra) in dilute anisotropic alloys is not a simple undertaking, and successful methodologies are not well developed. Our original approach started with the accurate calculations of the lattice structure of the dilute alloys using a disordered supercell. However, to continue and use these results to determine the phonon spectra, especially for our dilute anisotropic alloys, is too computationally demanding. Instead, effective medium approaches must be used. One such approach, the virtual crystal approximation (VCA), is well known for alloy calculations, but it does not work well for non-isoelectronic alloying elements like Al and Fe [40]. Another such approach is the coherent potential approximation (CPA), which works best for isotropic lattices [84] but not for highly anisotropic lattices like the delafossites, and, in the case of dilute alloys, it is computationally demanding $[41,85]$. Based on these issues, we do not use VCA or CPA to model the vibrational modes of our dilute anisotropic delafossite alloys. In principle, the calculation of the vibrational frequencies can be done based on the supercell results; however, these calculations are only 


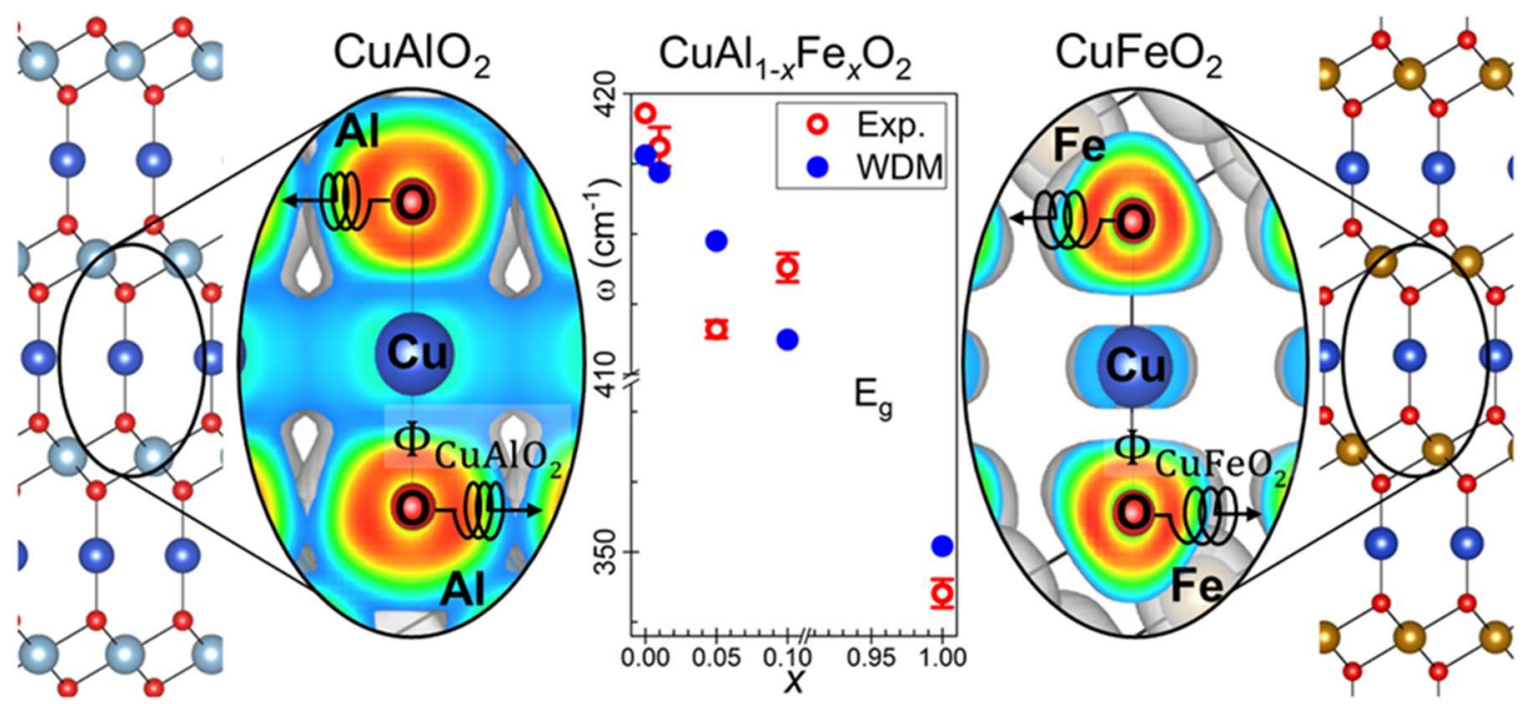

Figure 3.3.: Starting from the left and right edges, the supercells of $\mathrm{CuAlO}_{2}$ and $\mathrm{CuFeO}_{2}$ parent compounds are shown, with zoomed in images showing their modeled electron localization. These models allow the calculation of the wavenumber of the $\mathrm{E}_{\mathrm{g}}$ phonon mode for dilute $\mathrm{CuAl}_{1-x} \mathrm{Fe}_{x} \mathrm{O}_{2}$ alloys, using a weighted dynamical matrix (WDM) approach. Center we compare $\mathrm{E}_{\mathrm{g}}$ values for WDM approach to experiment.

tractable in the case of the parent structures, $\mathrm{CuAlO}_{2}$ and $\mathrm{CuFeO}_{2}$, at the endpoints. Again, for the alloys, especially the dilute alloys, the very large supercells make the calculations too computationally demanding. For these reasons, we developed a new effective-medium approach using a weighted dynamical matrix (WDM), based on modifying the masses and interatomic force constants after calculation of the HellmannFeynman forces [47] for parent compounds $\mathrm{CuAlO}_{2}$ and $\mathrm{CuFeO}_{2}$. There have been several attempts to consider mass and force-constant disorder in random alloys [42,44-46,86-89]. The approach presented here is straightforward and computationally efficient and works well for dilute anisotropic $\mathrm{CuAl}_{1-x} \mathrm{Fe}_{x} \mathrm{O}_{2}$ alloys.

\subsubsection{Weighted Dynamical Matrix (WDM) Approach:}

To find the phonon modes, one needs to construct the dynamical matrix. To do so, $\boldsymbol{r}_{l i}$ The ith atom's deviation in the supercell's lth unit cell from the equilibrium position is introduced. Also, the secondorder force constants for the $l i-l^{\prime} i^{\prime}$ atom pair denoted as $\Phi_{\alpha \beta}\left(0 i, l^{\prime} i^{\prime}\right.$ ) (with $\alpha$ and $\beta$ indices for coordinates) are calculated. Then, the dynamical matrix $\mathbf{D}(\mathbf{q})$ at the wavevector $\mathbf{q}$ is constructed as follows:

$\mathrm{D}_{i i^{\prime}}^{\alpha \beta}(\mathbf{q})=\frac{1}{\sqrt{m_{i} m_{i^{\prime}}}} \sum_{l^{\prime}} \Phi_{\alpha \beta}\left(0 i, l^{\prime} i^{\prime}\right) \exp \left[i \mathbf{q} \cdot\left(\mathbf{r}_{l^{\prime} i^{\prime}}-\mathbf{r}_{0 i}\right)\right]$, 


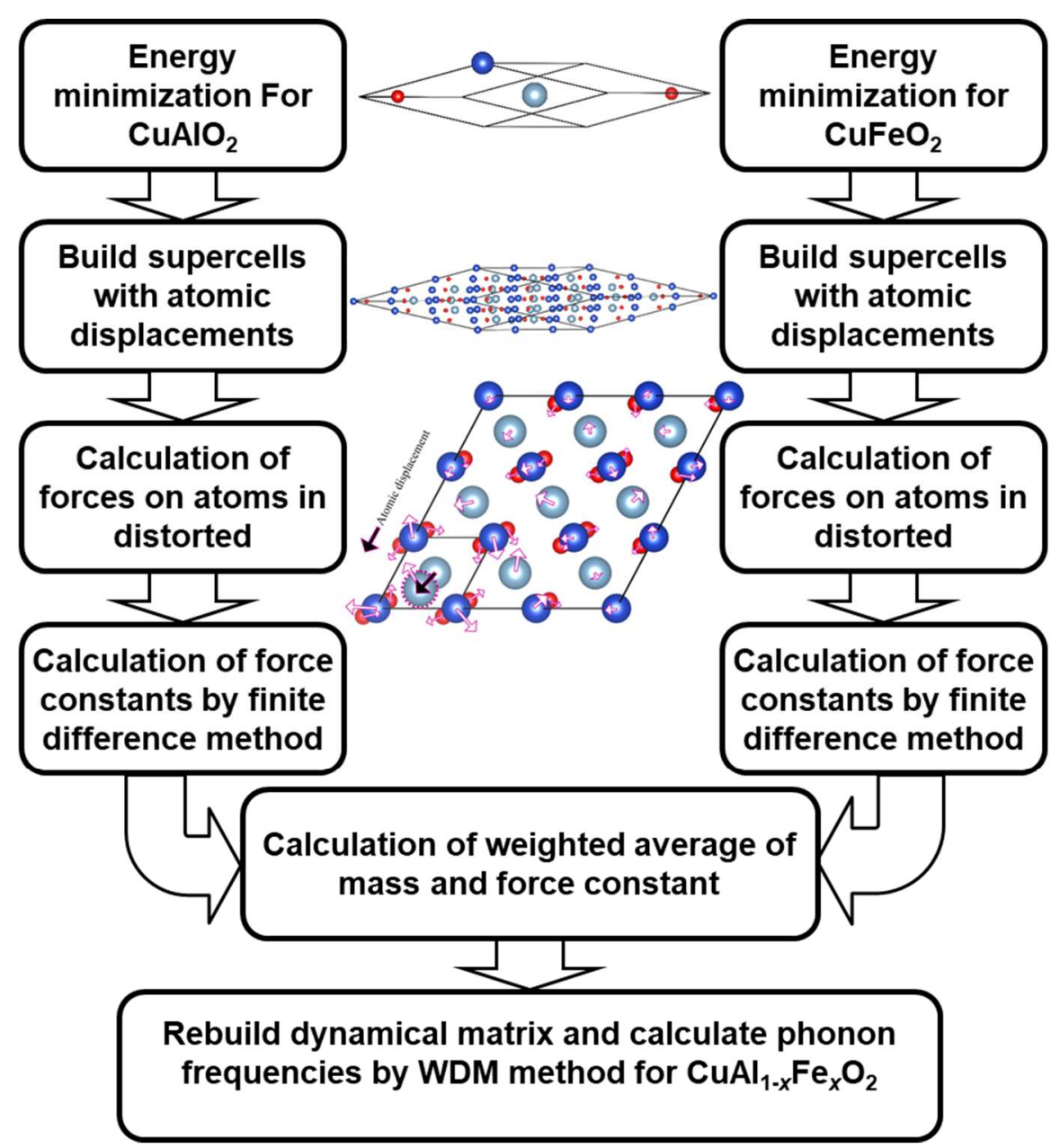

Figure 3.4.: Flow chart of WDM approach to calculate the alloys phonon modes using the computed results of the parent compounds.

where $m_{i}$ is the mass of the $i^{\text {th }}$ atom. Phonon frequencies $\omega(\mathbf{q}, \kappa)$ and mode eigenvectors $\chi(\mathbf{q}, \kappa)$ at $\mathbf{q}$, where $\kappa$ is the band index, are obtained by solving the eigenvalue equation:

$\mathbf{D}(\mathbf{q}) \chi(\mathbf{q}, \kappa)=[\omega(\mathbf{q}, \kappa)]^{2} \chi(\mathbf{q}, \kappa)$.

This proposed approach is tested and compared to our experimental results for $\mathrm{CuAl}_{1-x} \mathrm{Fe}_{x} \mathrm{O}_{2}$. To calculate the phonon modes for these alloy samples, first, the Hellmann-Feynman forces of the parent structures $\left(\mathrm{CuAlO}_{2}\right.$ and $\left.\mathrm{CuFeO}_{2}\right)$ for the atomic displacement of $0.01 \AA$ from their equilibrium positions are calculated by DFT calculations. Then from forces $\vec{F}_{\mathrm{CuAl}_{2}}$ and $\vec{F}_{\mathrm{CuFeO}_{2}}$ the force constants $\Phi_{\mathrm{CuA}_{2}}$ and $\Phi_{\mathrm{CuF} \quad 2}$ are calculated. The weighted dynamical matrix is constructed as follows:

$\overline{\mathrm{D}}_{i i^{\prime}}^{\alpha \beta}(\mathbf{q})=\frac{1}{\sqrt{m_{i} m_{i^{\prime}}}} \sum_{l^{\prime}} \bar{\Phi}_{\alpha \beta}\left(0 i, l^{\prime} i^{\prime}\right) \exp \left[i \mathbf{q} \cdot\left(\mathbf{r}_{l^{\prime} i^{\prime}}-\mathbf{r}_{0 i}\right)\right]$ 
where $\bar{\Phi}=x \Phi_{\mathrm{CuFe}_{2}}+(1-x) \Phi_{\mathrm{CuAlO}_{2}}$ refers to the weighted force constant, where $x$ refers to $\mathrm{Fe}$ concentration. Also, we consider a pseudo-atom instead of an M-site atom $\left(\mathrm{CuMO}_{2}\right)$ with mass as $m_{\mathrm{M}}=$ $x m_{F e}+(1-x) m_{A l}$ in Equation (3.16). Figure 3.4 shows a flow chart of the step by step procedure to calculate any percentage of alloy using the parent compounds computed results.

The advantage of our approach is that it can be easily used to estimate the interatomic force constants. Importantly, any percentage of dilute alloys can be considered, unlike the approach using a disordered supercell, which requires a huge supercell to represent a dilute alloy. However, there are some limitations to this approximation. First, by replacing Al with Fe, the Brillouin zone volume decreases, but this is not considered in the weighted dynamical matrix. Thus, we assume that this decrease does not influence the systems with dilute dopants. Also, in this method, we consider a pseudo-atom M instead of $\mathrm{Al}$ and Fe. Then, in states' projected density, the partial density of states from the individual $\mathrm{Al}$ and $\mathrm{Fe}$ atoms cannot be identified; only the constructed pseudo-atom $\mathrm{M}$ is available. This lack of identification implies that we do not consider the effect of Fe-alloying on the electronic dielectric permittivity or the Born-effective charges, which account for the long-range interaction of the macroscopic E-field induced by the polarization of collective ionic motions near the $\Gamma$-point.

Similar approaches, using only mass renormalization [90-92] or mass and force-constant renormalization, have been developed previously $[42,86-88]$. By considering only the weighted average mass, the phonon modes' decrease does not agree well with experimental data. This highlights the fact that the simple weighted average mass alone does not work well due to strain. The improvements for weighted averaging the interatomic force constants along with the masses are necessary.

Detailed features of our calculations include: interatomic force constants calculated for parent compounds $\mathrm{CuAlO}_{2}$ and $\mathrm{CuFeO}_{2}$ primitive unit cells (relaxation done with $8 \times 8 \times 8 \Gamma$-centered $k$-point mesh and kinetic energy cut-off increased to $600 \mathrm{eV}$ for both structures); supercells of $3 \times 3 \times 3$ with $0.01 \AA$ displacement are created [93] then forces (with $4 \times 4 \times 4 \Gamma$-centered $k$-point mesh) and Born-effective charges and electronic dielectric permittivity (with $16 \times 16 \times 16 \Gamma$-centered $k$-point mesh) are calculated with VASP. 


\subsection{Challenges of the Band Structure Calculation:}

The calculation of the electronic band structure and the resultant optical absorption for pure nonalloyed delafossites is already challenging. Density functional theory (DFT), using local density (LDA) and generalized gradient (GGA) approximations, successfully predicts the crystal geometry of the insulators. However, they usually fail to predict the insulating band structure of transition-metal oxides such as $\mathrm{CuAlO}_{2},[3,4,94,95]$ because they underestimate $d$-orbital correlations. [96] This can be overcome by including a Hubbard on-site Coulomb interaction $(\mathrm{DFT}+U)[10,76]$ or Green's function with Coulomb screening (GW), [34,97] both of which are more suitable for calculations of $d$ orbitals. $[75,80,98]$ Calculations using LDA/GGA $+U$ correct the energy of the $d$ orbitals through the $U$ parameter, which is itself not self-interaction free. These oxides exhibit strong polaronic (large polaron constant: $\alpha_{\mathrm{p}}, \alpha_{\mathrm{p}} \sim 1$ [99]) and excitonic effects (exciton binding energy of $\sim 0.5 \mathrm{eV}$ [97]) that can be modeled in GW calculations with a many-body quasiparticle approach. Alternatively, hybrid functional calculations can mostly fix the selfinteraction problem, improve the treatment of localized states and better predict the bandgap. [100] However, hybrid functionals are more computationally demanding than $\mathrm{DFT}+U$ calculations for delafossites, and these demands are greater still when extending the calculations to dilute alloys that require large supercells not one unit cell. For example, one Fe atom in a $3 \times 3 \times 3$ supercell is $4 \%$ Fe concentration $\left(\mathrm{CuAl}_{0.96} \mathrm{Fe}_{0.04} \mathrm{O}_{2}\right)$, whereas changes are expected observed for dilute concentrations at and below 1\%. For practical reasons, taking a GGA $+U$ approach for dilute alloys of $\mathrm{CuAl}_{1-x} \mathrm{Fe}_{x} \mathrm{O}_{2}$ will handle the charge distribution of the $d$ orbitals and make use of well-established values of $U$ [98] and can be applied across a wide concentration range.

\subsubsection{Calculation of the Electronic Band Structure:}

Calculations are performed within the DFT with a plane-wave basis set, as implemented in VASP [77,101,102]. A Perdew-Burke-Ernzerhof (PBE) [75] generalized gradient approximation (GGA) exchange-correlation functional [75] and projector-augmented wave pseudopotentials [80] with the valence-electron configurations $3 p^{6} 3 d^{10} 4 s^{1}, 3 p^{6} 3 d^{7} 4 s^{1}, 3 s^{2} 3 p^{1}$, and $2 s^{2} 2 p^{4}$ for $\mathrm{Cu}, \mathrm{Fe}, \mathrm{Al}$, and $\mathrm{O}$. A simplified 
rotationally invariant $\mathrm{PBE}+U$ approach [103] is taken with an on-site Coulomb parameter $U=4.0 \mathrm{eV}$, as suggested by Jain et al. . [98] for both $\mathrm{Fe}$ and $\mathrm{Cu}$. Also, spin polarization for Fe is included. The relaxation method used leaves the supercell volume, positions, and cell shape free to change. A $3 \times 3 \times 3$ supercell is constructed from the rhombohedral structure $(\mathrm{R} \overline{3} \mathrm{~m})$ primitive unit cell of the $\mathrm{CuAlO}_{2}$ with 108 atoms to be able to calculate the comparable dilute alloy structures. There are many possible configurations for $\mathrm{Fe}$ substituting for Al, but most of them can be ruled out by symmetry considerations, which is done by siteoccupation disorder SOD code [81]. The relaxation of this disordered supercell is done with a $4 \times 4 \times 4 \Gamma$ centered $k$-point grid. The energy cut-off $600 \mathrm{eV}$ for wave functions was employed for calculations. Primitive supercell calculations are used for the electronic band structure of dilute alloys. The band structure for a supercell is more complicated because of the uncertainty in the calculated wave functions. To simplify the alloyed supercell structure to the primitive representation, we use the Fold2Bloch code [104,105], which is based on a Bloch spectral density. The color spectrum from white to dark red is associated with the spectral weight from 0.0 to 1.0 , which is determined by both the magnitude of the corresponding Bloch character and degeneracy.

\subsection{Summary and Conclusion:}

In this chapter, the theory of the DFT is explained. The challenges of performing calculations for strongly correlated materials are addressed. The lattice parameters calculation and comparison of their percentage increase with the experimental results and the Vegard's law are used to optimize the Hubbard correction $U$. Furthermore, the challenges of phonon calculation for dilute anisotropic magnetic alloys of $\mathrm{CuAl}_{1-x} \mathrm{Fe}_{x} \mathrm{O}_{2}$ is explained in detail, and a weighted dynamical matrix approach is introduced. This approach is computationally efficient, applicable to a range of alloying elements, and computable for any alloy concentration percentage. Moreover, the challenges of the electronic band structure of $\mathrm{CuAlO}_{2}$ is explained. The necessary adopted computational method and parameters are presented. 


\section{Chapter 4}

\section{Variation of Magnetic Properties of Delafossite Alloys:}

\subsection{Overview:}

In this chapter, the temperature $(T)$ and magnetic field $(H)$ dependent magnetic properties of the $\mathrm{CuAl}_{1-x} \mathrm{Fe}_{x} \mathrm{O}_{2}$ alloys are presented that are measured using the vibrating sample magnetometry (VSM) and the electron spin resonance (ESR) to evaluate if the dilute alloys are room temperature ferromagnets. The Curie-Weiss and modified Brillouin functions for $T$ and $H$ dependent measurements, respectively. The Zeeman theory is used to find the g-factor from the ESR spectrum, see Figure 4.1.

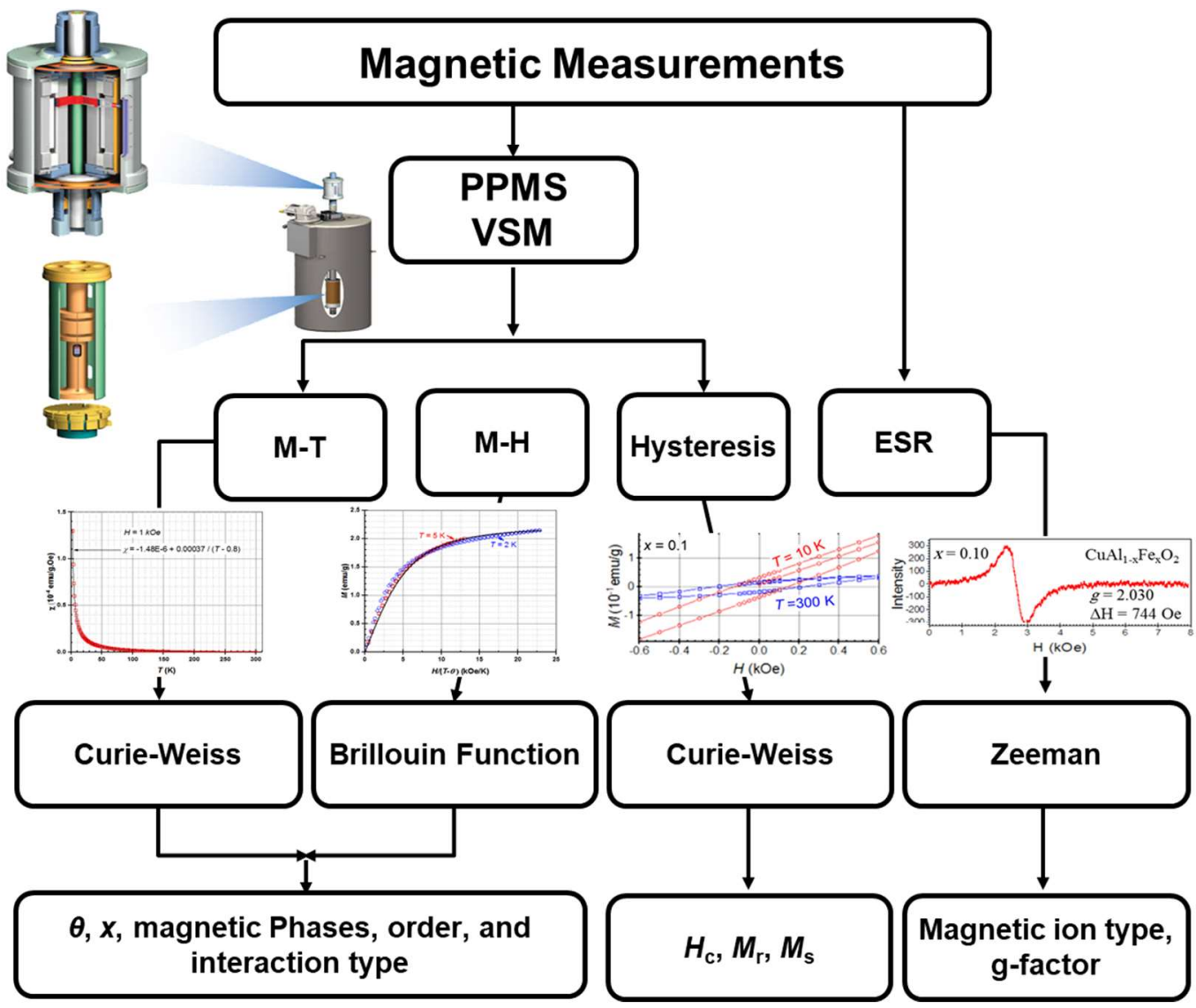

Figure 4.1: Flowchart of the magnetic measurements performed on $\mathrm{CuAl}_{1-x} \mathrm{Fe}_{x} \mathrm{O}_{2}$ powders and the theoretical fit used to study the magnetic properties. 


\subsection{Introduction:}

Technologically, $\mathrm{CuAlO}_{2}$ alloyed with $\mathrm{Fe}$ is of interest for at least two reasons: First, the control of the hole concentrations has led to the investigation of its thermoelectric properties for the energy conversion process after Park et al. [107] showed improvements in the thermoelectric properties of $\mathrm{CuAlO}_{2}$ by doping with $\mathrm{Fe}^{3+}$ at the $\mathrm{Al}^{3+}$ site; second, $\mathrm{CuAlO}_{2}$ doped with transition metal ions (V, $\mathrm{Cr}, \mathrm{Mn}, \mathrm{Fe}, \mathrm{Co}$, and Ni) may be a dilute magnetic semiconductor (DMS) with potential spintronic applications. Kizaki et al. [53] designed transparent and half-metallic DMS based on $\mathrm{CuAlO}_{2}$ from the first-principles calculations by using the KKR-CPA-LSDA method for V, Cr, Mn, Fe, Co, and Ni alloys. They calculated the exchange
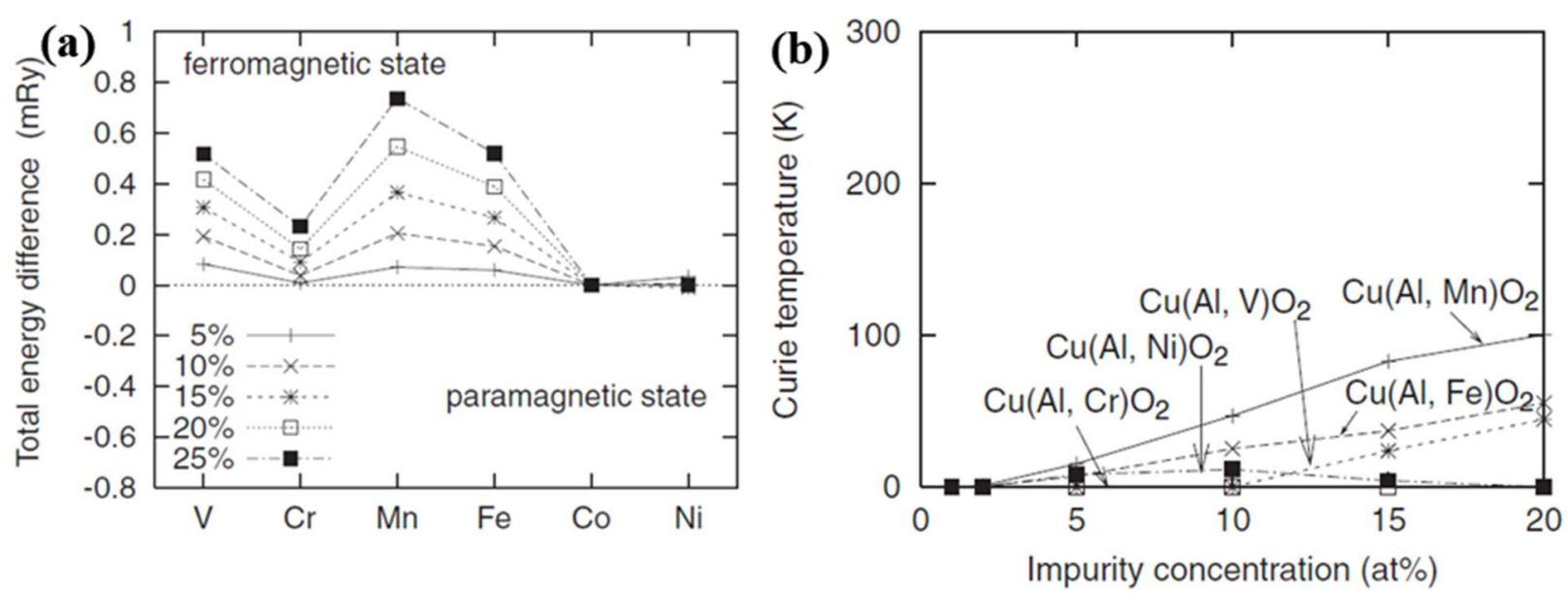

Figure 4.2: (a) Stability of the ferromagnetic state in $\mathrm{Cu}(\mathrm{Al}, \mathrm{M}) \mathrm{O}_{2}$ for $\mathrm{M}=\mathrm{V}, \mathrm{Cr}, \mathrm{Mn}, \mathrm{Fe}, \mathrm{Co}$, and Ni. Total energy difference per unit cell between the ferromagnetic state and the paramagnetic state are plotted. Concentrations of alloys are $5,10,15,20$, and $25 \%$. Positive energy difference means that the ferromagnetic state is more stable than the paramagnetic state. (b) Calculated $T_{\mathrm{C}}$ of $\mathrm{Cu}(\mathrm{Al}, \mathrm{V}) \mathrm{O}_{2}, \mathrm{Cu}(\mathrm{Al}, \mathrm{Cr}) \mathrm{O}_{2}, \mathrm{Cu}(\mathrm{Al}, \mathrm{Mn}) \mathrm{O}_{2}, \mathrm{Cu}(\mathrm{Al}, \mathrm{Fe}) \mathrm{O}_{2}$, and $\mathrm{Cu}(\mathrm{Al}, \mathrm{Ni}) \mathrm{O}_{2}$ within Monte Carlo Approximation as a function of the concentration of $\mathrm{V}, \mathrm{Cr}, \mathrm{Mn}, \mathrm{Fe}$, and $\mathrm{Ni}$, respectively. [53]

interaction between TM ions in $\mathrm{CuAlO}_{2}$-based DMS. For $\mathrm{Cu}(\mathrm{Al}, \mathrm{TM}) \mathrm{O}_{2}$, it is found from the total energy difference that almost all of the systems show ferromagnetism. In Figure 4.2 (a), the energy difference is plotted for all of the transition metal alloys. Ferromagnetism is the stable phase for all of them. In Figure 4.2 (b), the $T_{\mathrm{c}}$ is shown for all of the alloys using Monte Carlo approximation, which has a linearly increasing relationship to the alloy concentration up to concentrations of $20 \%$. This is a property of ferromagnetism. 
Previous studies by Park et al. [107] of the $\mathrm{CuAl}_{1-x} \mathrm{Fe}_{x} \mathrm{O}_{2}$ system did not include any magnetic measurements. Zhang et al. [108] reported magnetic measurements in $\mathrm{CuAl}_{1-x} \mathrm{Mn}_{x} \mathrm{O}_{2}(x=0.01,0.03,0.05$, and 0.07) and showed that fitting the data to the Curie-Weiss law $(\chi=C /(T-\theta))$ yielded negative $\theta$ and the Curie constant $C$ decreasing with increase in $x$, contrary to the calculations by Kizaki et al. [53], which predicted ferromagnetic interaction among $\mathrm{Mn}$ ions in $\mathrm{CuAl}_{1-x} \mathrm{Mn}_{x} \mathrm{O}_{2}$. Magnetic measurements in undoped $\mathrm{CuAlO}_{2}$ by Luo et al. [106] showed a ferromagnetic signature in the samples, which was related to holes produced by $\mathrm{Cu}$ vacancies and oxygen interstitials. Transport measurements in $\mathrm{CuAl}_{1-x} \mathrm{Ni}_{x} \mathrm{O}_{2}$ showed that with increasing $x$, conductivity, Hall mobility, and hole concentration for the samples decreased, possibly due to $\mathrm{Ni}^{2+}$ substituting for $\mathrm{Cu}^{1+}$. But, no magnetic investigation of these samples of $\mathrm{CuAl}_{1-x} \mathrm{Ni}_{x} \mathrm{O}_{2}$ was reported in their work.

From the above survey, it is evident that there has been a lack of magnetic investigations of the doped $\mathrm{CuAlO}_{2}$ samples, and so the predictions of Kizaki et al. [53] remain mostly untested.

In this chapter, the results of detailed investigations of the magnetic properties of polycrystalline powder samples of $\mathrm{CuAl}_{1-x} \mathrm{Fe}_{x} \mathrm{O}_{2}(x=0.0,0.01,0.05$, and 0.1$)$ are reported. The new results related to the question of whether $\mathrm{CuAl}_{1-x} \mathrm{Fe}_{x} \mathrm{O}_{2}$ is a room temperature ferromagnet, as some recent papers have reported, are investigated. The measurements of magnetization $(M) v s$. temperature $(T)$ of the samples were carried out from 2 to $300 \mathrm{~K}$ and in magnetic fields $(H)$ up to $90 \mathrm{kOe}$. These measurements show that the Curie constant, $C$, does scale with $x$, but $\theta$ is negative, showing that the exchange interaction among the $\mathrm{Fe}^{3+}$ ions is antiferromagnetic. The same conclusion is drawn from the analysis of the isothermal $M$ vs. $H$ measurements. Also, down to $2 \mathrm{~K}$, any transitions have not been observed to an ordered magnetic state. These conclusions disagree with the predictions resulting from the calculations of Kizaki et al. [53] noted earlier. Details of our results, discussion, and analysis are presented below. Furthermore, the high-resolution hysteresis measurement results at $10 \mathrm{~K}$ and room temperature are presented. Major conclusions from these investigations reported here are that although $M$ and $\chi$ do very nearly with $x$, minute quantities of impurities such as the spinel phase $\mathrm{CuAl}_{2} \mathrm{O}_{4}$ and $\alpha-\mathrm{Fe}_{2} \mathrm{O}_{3}$, which are not easily detected in x-ray diffraction (XRD) 
studies of the samples, play essential roles in the measured properties. The presence of $\alpha-\mathrm{Fe}_{2} \mathrm{O}_{3}$, which is a weak ferromagnet for $T>T_{\mathrm{M}}=260 \mathrm{~K}$ but an antiferromagnet for $T<T_{\mathrm{M}}$ [110] is the likely source of observed hysteresis loops at room temperature. Details of these results are presented in the following pages.

\subsection{Experimental Details:}

The magnetic measurements were performed using a PPMS Evercool II system. This system allows the sample temperature to be varied from 1.8 to $400 \mathrm{~K}$ with $1 \%$ accuracy and the magnetic field from $\mathrm{H}=-90$ to $90 \mathrm{kOe}$ with an accuracy of 0.2 Oe. For our samples, our estimated error in $M / \mathrm{m}(\mathrm{emu} / \mathrm{g})$ is less than $0.1 \%$, and in $\chi(\mathrm{emu} /(\mathrm{g} \mathrm{Oe})$ is also less than $0.1 \%$.

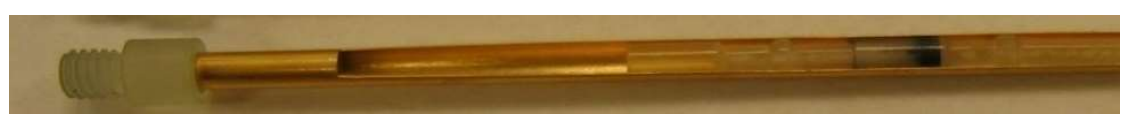

Figure 4.3: the picture shows the $\mathrm{CuAl}_{1-x} \mathrm{Fe}_{x} \mathrm{O}_{2}$ powder in the sample holder and snapped in the center of the brass half tube.

For the vibrating sample magnetometer (VSM) magnetic measurements, about $10 \mathrm{mg}$ of each powder sample is used. For each sample, the powder is compressed and sealed in a plastic tube sample holder (P/N 4096-388), shown in Figure 4.3. This sample holder snaps into a brass half tube that is appropriately positioned in the magnetic field. This sample is paramagnetic, and its susceptibility is known vs. the magnetic field. The calibration test is run before each measurement for the Palladium test sample. The small diamagnetic background related to the sample holder and the brass piece is measured (without the powder sample) and subtracted from the measured data measured for a powder sample associated with the powder samples. Note that it is crucial to make sure in VSM measurement, the sample is not able to move, and the particles should be tightly held in the sample holder.

\subsection{Theory of Paramagnetism:}

Paramagnetism is expected in atoms, molecules, and lattice defects possessing an odd number of electrons, free atoms, and ions with a partly filled inner shell, transition elements; ions isoelectronic with transition elements; rare earth and actinide elements, a few compounds with an even number of electrons, including 
molecular oxygen and organic biradicals, and metals. In the case of our interest material, the $\mathrm{Cu}^{2+}$ ion is a paramagnetic ion vs. $\mathrm{Cu}^{+}$is nonmagnetic.

Here, the physics necessary for modeling the paramagnets is explained. The magnetic moment of an atom is given by $\mu=-g \mu_{B} J$ where $J$ is the total angular momentum $J=L+S$ (orbital $L$ and $\operatorname{spin} S$ angular momenta), $g$ is the splitting factor ( $g$-factor), and $\mu_{\mathrm{B}}$ is the Bohr magneton (eh/2mc in CGS and $e \hbar / 2 m$ in SI). The g-factor for a free atom is formulated as follows $(g=2.0023 \sim 2.00$ for a free electron):

$g=1+\frac{J(J+1)+S(S+1)-L(L+1)}{2 J(J+1)}$

The energy levels of an atom with angular momentum $J$ in a magnetic field are:

$U=-\mu . B=m_{J} g \mu_{B}$

where $m_{J}$ is the azimuthal quantum number $\left(-J \leq m_{J} \leq J\right)$, which is called the Zeeman splitting. For a single spin with no orbital moment, $m_{J}= \pm 1 / 2$ and $\mathrm{g}=2$, and $U= \pm \mu_{B} B$. In this case, the equilibrium populations are defined as:

$\frac{N_{1}}{N}=\frac{\exp \left(\mu_{B} B / \tau\right)}{\exp \left(\mu_{B} B / \tau\right)+\exp \left(-\mu_{B} B / \tau\right)}$

$\frac{N_{2}}{N}=\frac{\exp \left(-\mu_{B} B / \tau\right)}{\exp \left(\mu_{B} B / \tau\right)+\exp \left(-\mu_{B} B / \tau\right)}$

where $\tau=k_{B} T, N_{1}, N_{2}$ are the populations of the lower and upper levels, and $N$ is the total number of magnetic atoms. The magnetic moment of the upper state along the field direction is - $\mu$, and of the lower state is $\mu$. The resultant magnetization for $N$ atoms per unit volume is as follows (with $x=\mu_{B} / k_{B} T$ ):

$M=\left(N_{1}-N_{2}\right) \mu=N \mu \frac{e^{x}-e^{-x}}{e^{x}+e^{-x}}=N \mu \tanh x \stackrel{x<<1}{\Longrightarrow} M=N \mu\left(\frac{\mu B}{k_{B} T}\right)$

In general, for a magnetic field, an atom with angular momentum quantum number $J$ has $2 J+1$ equally spaced energy levels, and the magnetization is given by: 
$M=N \mu J \mu_{B} B_{J}(x)\left(x=\frac{g J \mu_{B} B}{k_{B} T}\right)$

where the Brillouin function $B_{J}$ is defined as follows:

$B_{J}(x)=\frac{2 J+1}{2 J} \operatorname{ctnh}\left(\frac{(2 J+1) x}{2 J}\right)-\frac{1}{2 J} \operatorname{ctnh}\left(\frac{x}{2 J}\right)$

In magnetic-field dependent measurement, this equation is used for fitting our isothermal $M$ vs. $H$ data. Equation (4.6) is a particular case of (4.5) for $J=1 / 2$.

If $x=\mu B / k_{B} T<<1$, the susceptibility is simplified to the following:

$\chi=\frac{M}{B}=\frac{N J(J+1) g^{2} \mu_{B}^{2}}{3 k_{B} T}-\frac{N p^{2} \mu_{B}^{2}}{3 k_{B} T}=\frac{C}{T}$

Where $C$ is the Curie constant. Equation (4.8) is known as the Curie law, and (4.9) is known as the CurieWeiss law [111].

In the paramagnetic phase, applied field $\mathrm{B}_{\mathrm{a}}$ causes a finite magnetization, which can lead to a finite exchange

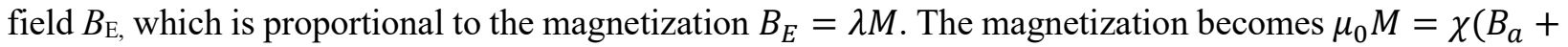
$B_{E}$ ). By replacing the magnetic field in Equation (9), obtain the following equation is obtained:

$\chi=\frac{M}{B_{a}}=\frac{C}{T-\theta}$

This equation is called the Curie-Weiss law. For the case of our dilute alloys with $\mathrm{Fe}^{3+}$ magnetic ions, the data should be fitted with the above equation.

The exchange field represents the exchange interaction, which is included in $\theta$. According to the Heisenberg model, the exchange energy of two electrons is $-2 J_{\mathrm{s}_{1}} . \mathrm{s}_{2}$. Then, if the $\mathrm{z}$ number of nearest neighbors is considered for an atom, the exchange energy will be $-2 z J_{\mathrm{s}_{1}} . \mathrm{s}_{2}$. At any specific temperature, thermal energy is $3 \mathrm{k}_{\mathrm{B}} T$. At $T=\theta$, the exchange energy is suppressed by the thermal energy $3 \mathrm{k}_{\mathrm{B}} \theta$. Therefore, after a simple calculation, the $T_{\mathrm{c}}$ relationship to $J$ can be estimated as follows [111]:

$J=\frac{3 k_{B} \theta}{2 z S(S+1)}$ 
From Equation (11), it is understood that by fitting to the Curie-Weiss law, the exchange integrals strength and the type of exchange can be estimated. If $\theta<0$ the interaction is antiferromagnetic and if $\theta>0$ is ferromagnetic [111].

\subsection{Magnetic Properties of Alloys:}

\subsubsection{Temperature Dependence of Magnetization:}

The temperature dependence of the magnetic susceptibility $\chi=M / H(M$, magnetization, and $H$, applied magnetic field) for $H=1 \mathrm{kOe}$ is shown in Figure 3 for the two undoped samples of $\mathrm{HP}$ and $\mathrm{LP} \mathrm{CuAlO}_{2}$ (HP and LP refer to the purity of the $\mathrm{Cu}_{2} \mathrm{O}$ precursor). (In this work, the samples were cooled in zero applied magnetic fields from $300 \mathrm{~K}$ to $2 \mathrm{~K}$, followed by application of a measuring magnetic field and taking the data with increasing temperatures after stabilizing the temperature at each measuring temperature).

From the plots in Figure 4.4, it is evident that for undoped $\mathrm{CuAlO}_{2}, \chi_{\mathrm{LP}}>\chi_{\mathrm{HP}}$ at all temperatures. This is expected since the concentration of $\mathrm{Cu}^{2+}$ ions associated with the surface $\mathrm{Cu}^{2+}$ that is (discussed in chapter 2), and the possible spinel phase is higher in the LP sample. The following equation is used to fit the theory to $\chi$ vs. $T$ data, which included the temperature-independent term, called the modified Curie-Weiss law given by:

$$
\chi=\chi_{0}+\frac{C}{(T-\theta)}
$$


In Equation 4.11, $\chi_{0}$ is the temperature-independent component containing contributions from diamagnetism of the ions and Van Vleck susceptibility, $C=N \mu^{2} / 3 k_{B}$, where $N$ is the number of magnetic ions per gram and $k_{B}$ Boltzmann's constant. Note that $N=x N_{A} / M W$ is used, where $N_{A}$ is Avogadro's constant, $M W$ is the sample's molecular weight, $x$ is the concentration of $\mathrm{Cu}^{2+}$ ions for the undoped samples, and $\mathrm{Fe}^{3+}$ ions for the doped samples and $\theta$ is the Curie-Weiss temperature, which is proportional to exchange integral, $J$, between $\mathrm{Fe}^{3+}$ ions. $\chi_{0}$ is determined from the plot of $\chi v s .1 / T$ using the $y$-intercept in the limit of $1 / T=0$. The fits of the data to Equation 4.11 are shown as solid lines in Figure 4.3, and the values of the fitted parameters $\left(\chi_{0}, C\right.$, and $\left.\theta\right)$ are listed in Table 4.1. $\mu=1.9 \mu_{B}$ is used for $\mathrm{Cu}^{2+}$ with $g=2.19$, as the typical average values for $\mathrm{Cu}^{2+}$ ions, and use $\mu^{2}=g^{2} \mu_{B}^{2} S(S+1)$ with $S=1 / 2$ and determine experimental values of $C=1.14 \times 10^{-4} \mathrm{emu} \cdot \mathrm{K} \cdot \mathrm{g}^{-1} \mathrm{Oe}^{-1}\left(0.99 \times 10^{-4} \mathrm{emu} \cdot \mathrm{K} \cdot \mathrm{g}^{-1} \mathrm{Oe}^{-1}\right)$ for LP (HP) samples. This analysis yields $x=$ 0.031 (0.028) for the $\mathrm{CuAl}_{2} \mathrm{O}_{4}$ impurity in the undoped samples [7].

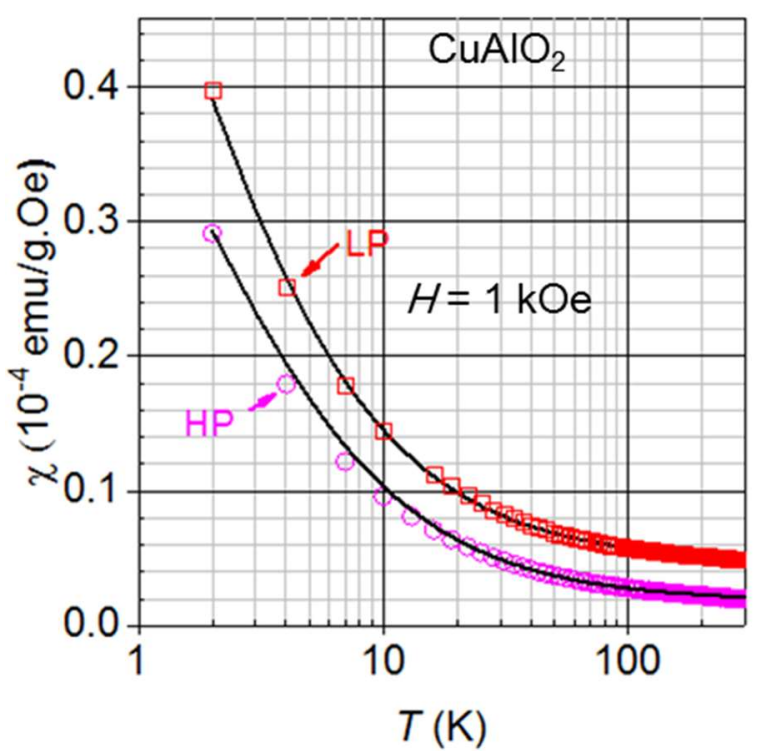

Figure 4.4: Magnetic susceptibility $\chi=M / H$ vs. $T$ is plotted for the high purity and low-purity $\mathrm{CuAlO}_{2}$ samples at $H=1 \mathrm{kOe}$. The solid lines are fits to the Curie-Weiss law (Eq. 12) with fitting parameters given in Table 4.1. 


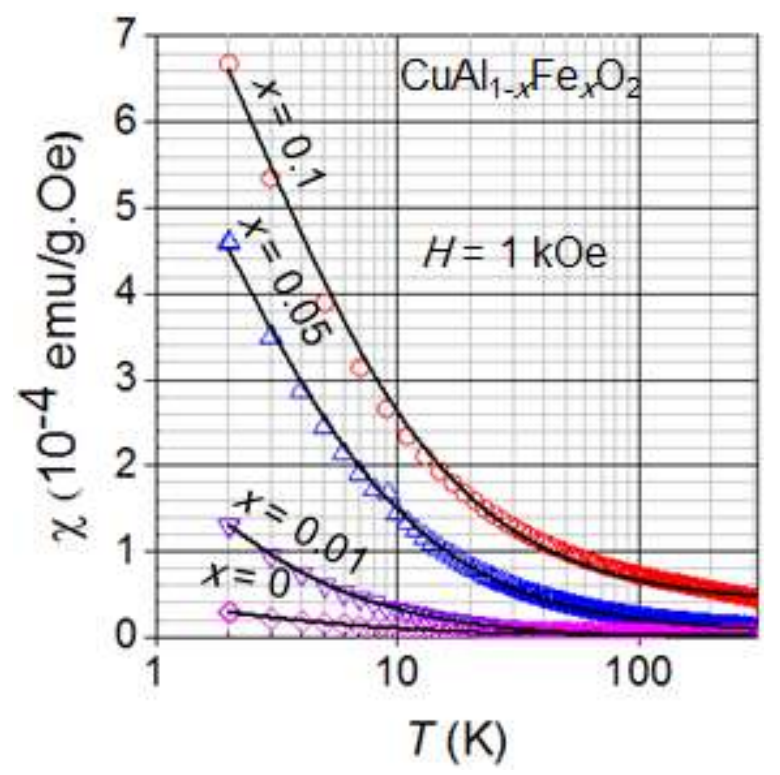

Figure 4.5: $M$ vs. $T$ plots for $\mathrm{CuAl}_{1-x} \mathrm{Fe}_{x} \mathrm{O}_{2}$ samples at $H=1 \mathrm{kOe}$. Symbols are experimental points and lines fits to the modified Curie-Weiss law (Eq. 12) with fitting parameters given in Table 4.1.

The temperature dependence of the magnetic susceptibility for the three samples of $\mathrm{CuAl}_{1-x} \mathrm{Fe}_{x} \mathrm{O}_{2}$ with nominal $x=0.01,0.05$, and 0.1 is shown in Figure 4.5. The data for the undoped HP sample is included for comparison purposes. The log-scale on the temperature axis is used to highlight the low-temperature variations since $\chi$ vs. $T$ has a rapid variation in this regime. Solid lines are fits to Eq. 12 with the fitting parameters given in Table 1 . As expected, $\chi$ qualitatively scales with the magnitude of $x$, and the temperature dependence of $\chi$ for the undoped $\mathrm{CuAlO}_{2}$ is comparatively negligible. Nevertheless, the contribution of $\chi$ of the undoped sample is subtracted from the magnitude of $\chi$ for $x=0.01,0.05,0.1$ samples to determine the magnitudes of $C$ and $\theta$. Assuming $S=5 / 2$ and $g=2$ (as is normally observed for $\mathrm{Fe}^{3+}$ ions) yields $x=$ $0.011,0.044$, and 0.086 for the nominal doping of $x=0.01,0.05$, and 0.1 , respectively. This comparison represents a reasonably good agreement. The sign of $\theta$ is negative for all samples implying that the exchange coupling between the nearest-neighbor $\mathrm{Fe}^{3+}-\mathrm{Fe}^{3+}$ ions is antiferromagnetic in nature. The observed increase in the magnitude of $\theta$ with an increase in $x$ is understandable because the probability of $\mathrm{Fe}^{3+}$ ions being nearest neighbors increases with an increase in $x$. Also, some clustering of the magnetic ions cannot be ruled out [7]. 


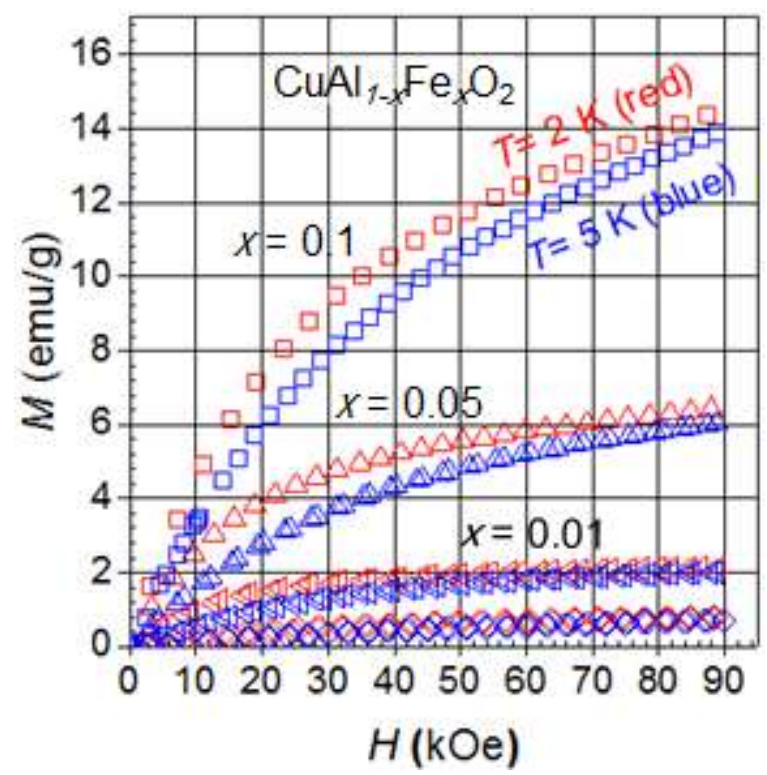

Figure 4.6: Data of $M v s . H$ are plotted for the $\mathrm{CuAl}_{1-x} \mathrm{Fe}_{x} \mathrm{O}_{2}$ samples with $x=0.0(\mathrm{HP}), 0.01,0.05$, and 0.1 for $T$ $=2 \mathrm{~K}$ (red) and $5 \mathrm{~K}$ (blue).

\subsubsection{Magnetic Field Dependence of Magnetization}

The isothermal magnetic field dependence of the magnetization was measured at select temperatures between 2 to $300 \mathrm{~K}$ at magnetic fields up to $90 \mathrm{kOe}$ (max. field for our PPMS Evercool II system). The $M$ vs. $H$ data at 2 and $5 \mathrm{~K}$ for samples with $x=0.0$ (HP), $0.01,0.05$, and 0.10 are plotted in Figure 4.6. These plots show how $M$ changes with $x$ and $H$. As expected from the $M v s . T$ measurements presented earlier, $M$ at a given $H$ increases with an increase in the Fe concentration and decrease in $T$.

To see how a given sample $M v s$. $H$ compares at different $T$, in Figure 4.7, we show the $M v s$. $H$ plots for the $x=0.01$ sample at $T=2,5,10$, and $20 \mathrm{~K}$. A simple check of this data indicates that $M v s . H$ does not simply scale as $H / T$ as expected for a simple paramagnet. Therefore, we analyze the data using the Brillouin function with $H /(T-\theta)$ scaling, as has been done for similar systems. The data of $M v s . H$ are fitted to the modified Brillouin function given by the following equation:

$M=x N g \mu_{B} S B_{S}(y), y=\frac{g \mu_{B} S H}{k_{B}(T-\theta)}$, with 
$B_{S}(y)=\frac{2 S+1}{2 S} \operatorname{coth}\left(\frac{2 S+1}{2 S} y\right)-\frac{1}{2 S} \operatorname{coth} \frac{1}{2 S} y$.
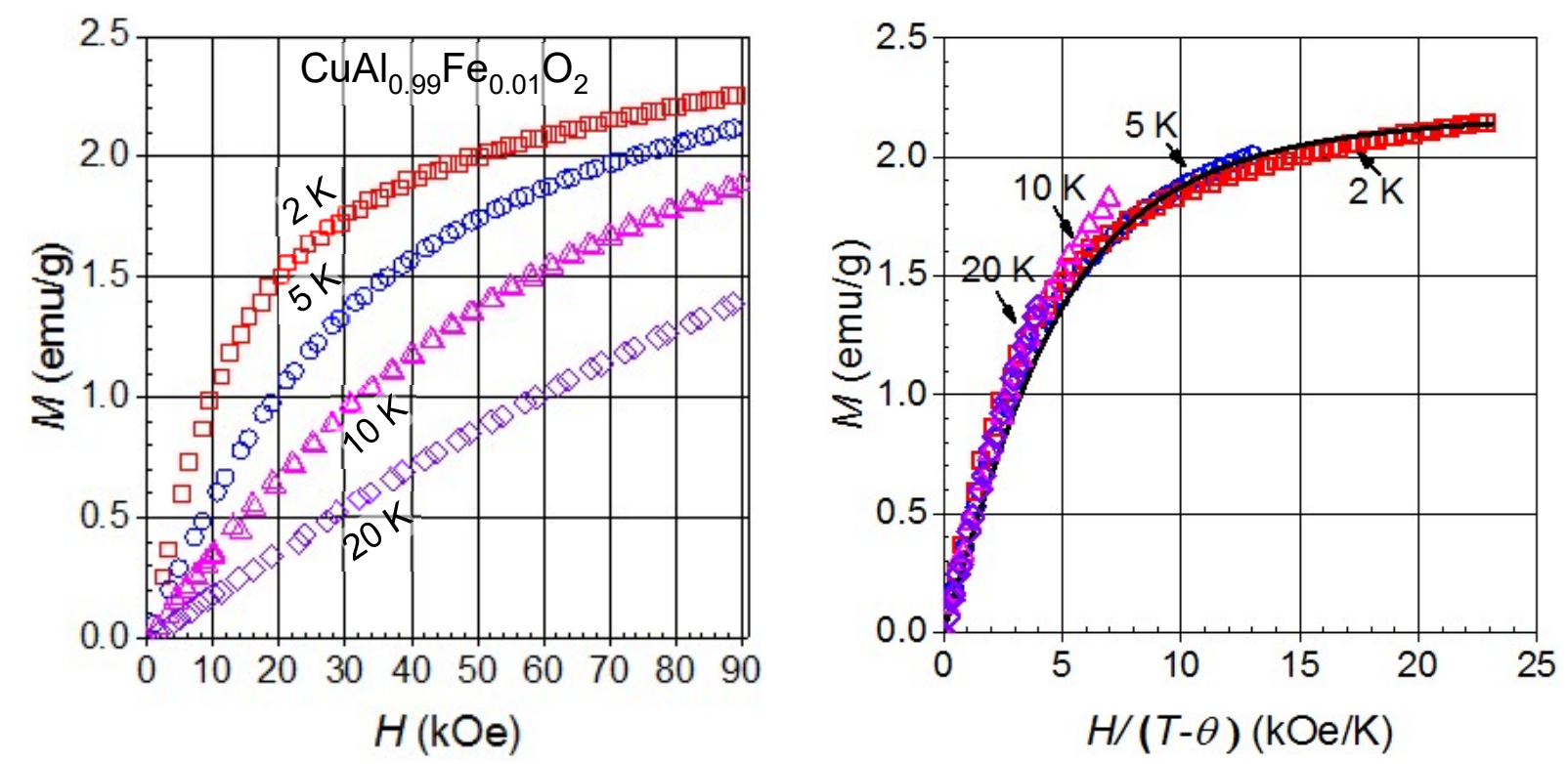

Figure 4.7: Top graph shows $M$ vs. $H$ for $\mathrm{CuAl}_{0.99} \mathrm{Fe}_{0.01} \mathrm{O}_{2}$ at $T=2,5,10$, and $20 \mathrm{~K}$. Bottom graph shows $M v s$. $H /(T-\theta)$ and black solid line shows the fit to the Eq. 14.

For fitting the $M v s$. $H$ data, $\theta$ was chosen so that the data at 2 and $5 \mathrm{~K}$ collapse to a single curve. Again, a negative $\theta$, characteristic of antiferromagnetic exchange coupling, is required for collapsing the data to a single curve. $M v s . H /(T-\theta)$ plotted for the $x=0.01$ sample is shown in Figure 4.6 with the solid line a fit to the modified Brillouin function (Eq. 13 and 14) with the magnitudes of the fitted parameters as follows: $x$ $=0.0097, \theta=-1.9 \mathrm{~K}$, and $M_{0}=2.18 \mathrm{emu} / \mathrm{g}$. Fits for the other samples using the data at 2 and $5 \mathrm{~K}$ are shown in Figure 10 with the magnitudes of the fitting parameters $x, \theta$, and $M_{0}$, listed in Table 4.1 [7]. 


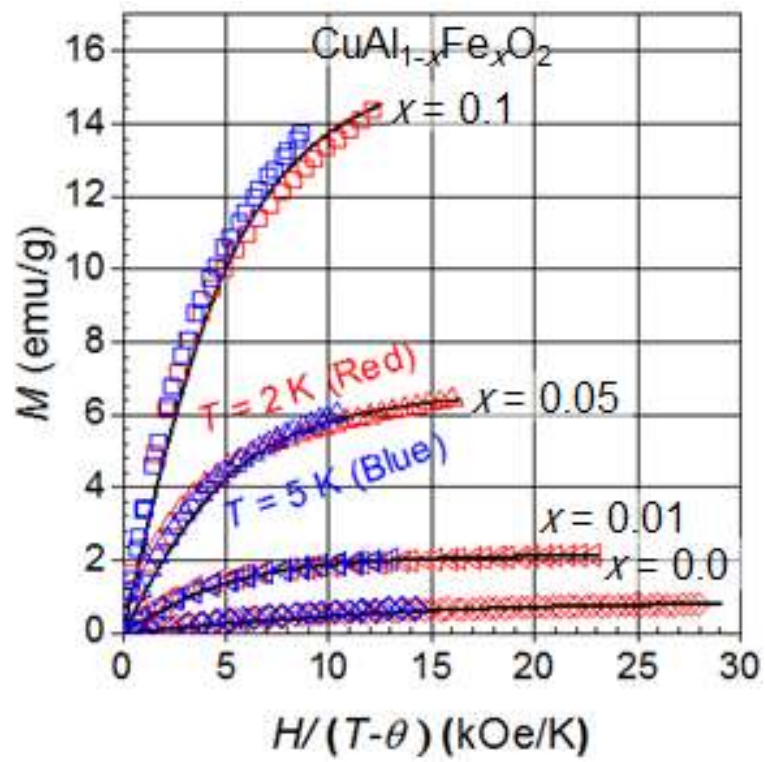

Figure 4.8: $M v s . H /(T-\theta)$ for $x=0.0,0.01,0.05$, and 0.1 at $T=2$ and $5 \mathrm{~K}$. Solid lines are fits to the Brillouin function (Eq. 13 and 14).

Next, the magnitudes of the parameters determined from the analysis of the $M$ vs. $T$ data are compared to the Curie-Weiss law, which follows from the Brillouin function in the limit of $y<<1$ and the Brillouin function variation often observed only at low $T$ and high $H$ (Figs. 4.6, 7, and 8). These parameters listed in Table 1 show overall good agreement between the parameters determined from the $M v s . T$ fits and $M v s$. $H$ fits. Note that $M_{0}=x N g \mu_{B} S$ is valid only for the $T=0 \mathrm{~K}$ limit, whereas our data could only be taken at $2 \mathrm{~K}$. Since experimental $M_{0}$ increases with a decrease in $T$ (see Figure 4.10), the magnitudes of $x$ determined from $M_{0}$ are somewhat underestimated. Therefore, measurements at still lower temperatures and higher magnetic fields are likely to provide even better agreement, as shown recently in another system [7]. 


\subsubsection{High-resolution magnetic hysteresis loops}

(a)

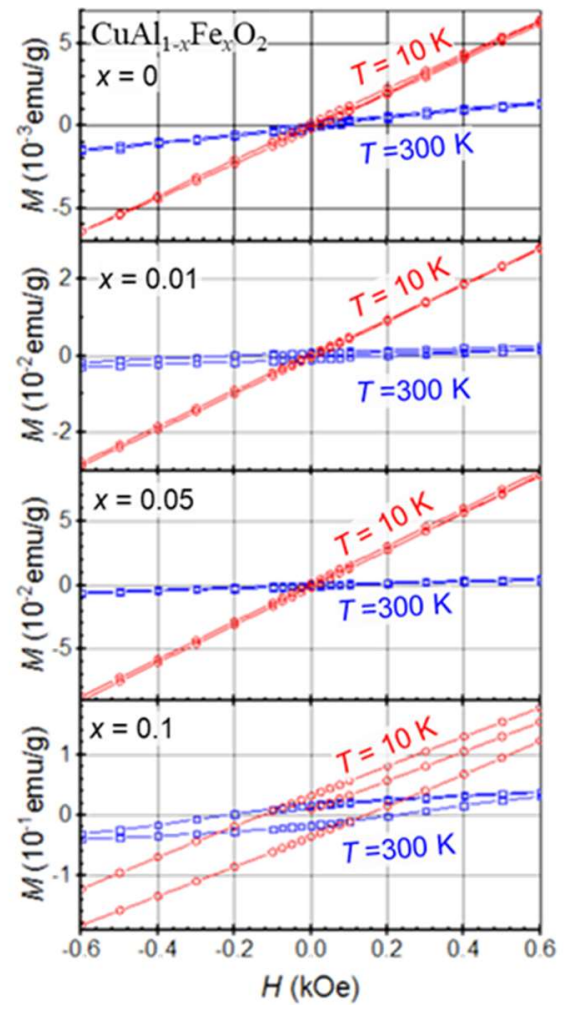

(b)

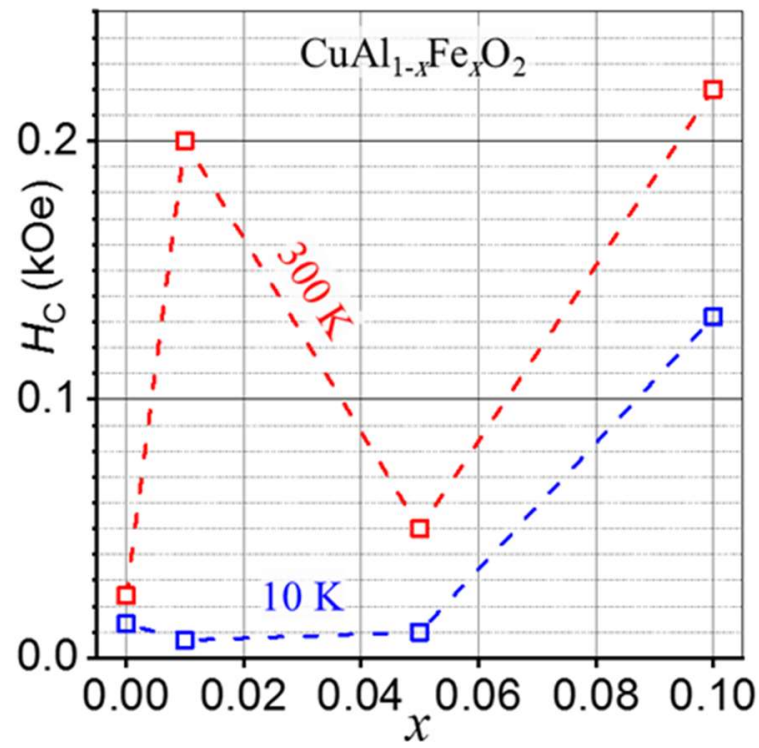

Figure 4.9: (a) Hysteresis loops are plotted for $x=0.0,0.01,0.05$, and 0.1 at $10 \mathrm{~K}$ and room temperature. (b) the coercive field obtained from the hysteresis loops are plotted vs. Fe concentration.

The high-resolution hysteresis loop measurements of $M$ vs. $H$ at 300 and $10 \mathrm{~K}$ are carried out to address the question of room-temperature ferromagnetism (RTFM) in the $\mathrm{CuAl}_{1-x} \mathrm{Fe}_{x} \mathrm{O}_{2}$ samples. The plots of $M$ vs. $H$ at 300 and $10 \mathrm{~K}$ for the low- $H$ region are shown in Figure 4.9 (a) for $x=0.0-0.10$ samples. The variation of coercivity $\left(H_{C}=\frac{H_{+}-H_{-}}{2}\right)[113]$ vs. $x$ measured at 300 and $10 \mathrm{~K}$ for all four samples is plotted in Figure 4.8 (b). For the $x=0.0$ sample, $H_{\mathrm{C}} \approx 0$ at both 300 and $10 \mathrm{~K}$. For the samples with $x=0.01$ and $0.05, H_{\mathrm{C}}=0$ at $10 \mathrm{~K}$ but significant $H_{\mathrm{C}} \sim 100 \mathrm{Oe}$ is observed at $300 \mathrm{~K}$. For the $x=0.10$ sample, $H_{\mathrm{C}} \sim 100$ Oe both at 10 and $300 \mathrm{~K}$. These results suggest that the samples of $\mathrm{CuAl}_{1-x} \mathrm{Fe}_{x} \mathrm{O}_{2}$ doped with $\mathrm{Fe}$ are ferromagnetic at 300 $\mathrm{K}$ as also reported by others $[53,109]$. However, $H_{\mathrm{C}} \sim 0$ at $10 \mathrm{~K}$ for the $x=0.01$ and 0.05 samples suggest that these observations are very likely due to hematite $\left(\alpha-\mathrm{Fe}_{2} \mathrm{O}_{3}\right)$ present in the samples as an impurity but not detected by XRD or XPS. This conclusion is based on the fact that $\alpha-\mathrm{Fe}_{2} \mathrm{O}_{3}$ is a weak ferromagnet above the Morin temperature $T_{M} \approx 260 \mathrm{~K}$, but it transforms to an antiferromagnet for $T<T_{M}[114,115]$. Therefore, 
$M$ vs. $H$ variation due to $\alpha-\mathrm{Fe}_{2} \mathrm{O}_{3}$ will show a hysteresis loop at $300 \mathrm{~K}$. However, $M$ vs. $H$ will be linear without hysteresis at $10 \mathrm{~K}$. Based on the observed magnitude of the weak ferromagnetic moment of 0.001 , 0.001 , and $0.02 \mathrm{emu} / \mathrm{g}$ at $300 \mathrm{~K}$ for the nominal $x=0.01,0.05$ and 0.10 samples of $\mathrm{CuAl}_{1-x} \mathrm{Fe}_{x} \mathrm{O}_{2}$, respectively, and comparison to $M_{S}=0.45 \mathrm{emu} / \mathrm{g}$ for a bulk sample of $\alpha-\mathrm{Fe}_{2} \mathrm{O}_{3}[111,115]$, the concentration of $\alpha-\mathrm{Fe}_{2} \mathrm{O}_{3}$ impurity in $\mathrm{CuAl}_{1-x} \mathrm{Fe}_{x} \mathrm{O}_{2}$ samples is estimated to be about $0.2 \%$ in the $x=0.01$ and 0.05 samples and about $4 \%$ in the $x=0.10$ samples. The source of this impurity is some unreacted $\alpha-\mathrm{Fe}_{2} \mathrm{O}_{3}$ since $\alpha-\mathrm{Fe}_{2} \mathrm{O}_{3}$ is one of the precursors used in synthesizing the $\mathrm{CuAl}_{1-x} \mathrm{Fe}_{x} \mathrm{O}_{2}$ samples. This evidence also explains why the concentration $x$ determined from the analysis of the magnetic data, particularly for the sample with nominal $x=0.1$, is smaller than the nominal value, as noted above. Perhaps temperatures higher than 1,100 ${ }^{\circ} \mathrm{C}$ during the synthesis of the samples are needed to eliminate this impurity. Regarding RTFM in the $\mathrm{CuAl}_{1-}$ ${ }_{x} \mathrm{Fe}_{x} \mathrm{O}_{2}$ samples, it is clear that the role of impurities needs to be carefully considered since, as noted here, these impurities levels are not detectable in standard XRD analysis of the samples. From the results presented here, we inferred that the pure samples of $\mathrm{CuAl}_{1-x} \mathrm{Fe}_{x} \mathrm{O}_{2}$ without impurities are likely not ferromagnetic at room temperatures [8]. 


\subsection{Electron Spin Resonance Measurement:}

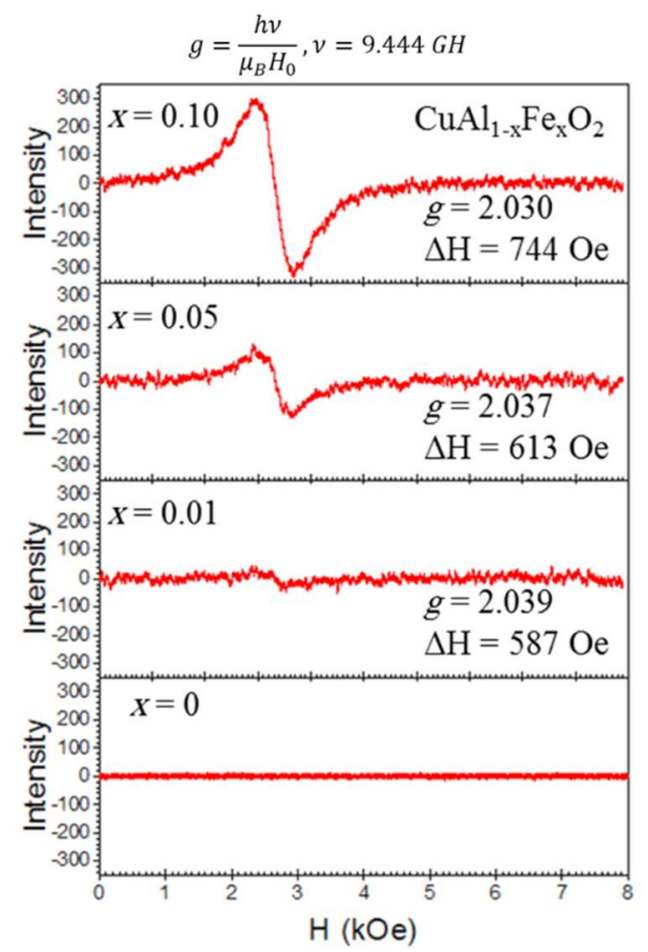

Figure 4.10: The ESR spectra for all four samples $\mathrm{CuAl}_{1-x} \mathrm{Fe}_{x} \mathrm{O}_{2}$ are plotted.

The electron spin resonance measurements are performed for the alloys at room temperature at $9.444 \mathrm{GH}$ frequency in the magnetic field up to $8 \mathrm{kOe}$, see Figure 4.10. In the $x=0$ samples, no resonance frequency was observed. However, for $x>0$, only one resonance frequency is observed. The resonance amplitude is increasing vs. $x$, which confirms the association of the resonance frequency to the iron content. Using $g=$

$\frac{h v}{\mu_{B} H_{0}}$ in the equation, the g-factor is found. The obtained values confirm that the magnetic ion is $\mathrm{Fe}^{3+}$. The $\mathrm{Fe}^{3+}$ g-factor can vary by the strength of interaction with other magnetic ions. In this case, the g-factor is small, and it shows that the magnetic ions are weakly interacting with each other.

\subsection{Conclusion:}

In this chapter, the data of the temperature and magnetic field dependence of the magnetization in the $\mathrm{CuAl}_{1-x} \mathrm{Fe}_{x} \mathrm{O}_{2}$ samples with $x=0.0,0.01,0.05$, and 0.1 are presented along with the analysis and discussion of the results. The results show that $\mathrm{Fe}$ is present as $\mathrm{Fe}^{3+}$ replacing $\mathrm{Al}^{3+}$ and the estimated value 
of $x$ using magnetic measurements is in good agreement with the nominal values of $x$. These results also show the absence of a magnetic phase transition in the Fe-doped samples at least down to $2 \mathrm{~K}$ and that $\mathrm{Fe}^{3+}$ $\mathrm{Fe}^{3+}$ exchange-coupling is antiferromagnetic in nature. Because these results do not agree with the theoretical prediction of the ferromagnetic $\mathrm{Fe}^{3+}-\mathrm{Fe}^{3+}$ exchange coupling and the possible presence of a Curie temperature increasing with an increase in $x$, a revised look at the relevant theoretical analysis is warranted. Moreover, by the electron spin resonance measurement, we confirmed that the paramagnetism is coming from the $\mathrm{Fe}^{3+}$ as no resonance was observed for the unalloyed sample. The hysteresis loops and weak ferromagnetism observed at $300 \mathrm{~K}$ for the Fe-doped samples are shown to be likely due to the presence of hematite as an impurity in the samples. This impurity possible results from some un-reacted hematite, which is one of the precursors used in the solid-state synthesis of the $\mathrm{CuAl}_{1-x} \mathrm{Fe}_{x} \mathrm{O}_{2}$ samples. Since the small level of this impurity is not detected in the standard XRD analysis of the samples, magnetic measurements, as reported here, are essential to detect this impurity. Because hematite is a weak ferromagnet at room temperature, the presence of RTFM in the impurity-free samples of $\mathrm{CuAl}_{1-x} \mathrm{Fe}_{x} \mathrm{O}_{2}$ samples is highly unlikely.

Table 4.1. Fitting parameters of the $M v s . T$ data fit to the modified Curie-Weiss law (Eq. 13) and the $M v s . H$ variations (Eq. 13 and 14). For the LP and HP samples, the magnitudes of $x$ are determined using $S=1 / 2$ for $\mathrm{Cu}^{2+}$. For the Fe-doped samples, calculations of $x$ are based on using $S=5 / 2$ for $\mathrm{Fe}^{3+}$.

\begin{tabular}{|c|c|c|c|c|c|c|c|}
\hline & \multicolumn{4}{|c|}{$M v s . T$} & \multicolumn{3}{c|}{$M v s . H$} \\
\hline Sample & $\chi_{0}$ & $C$ & $\theta$ & $x$ & $M_{0}$ & $\theta$ & $x$ \\
\hline & $\mathrm{emu} / \mathrm{g} \cdot \mathrm{Oe})$ & $\begin{array}{c}\mathrm{emu} \cdot \mathrm{K} /(\mathrm{g} \cdot \mathrm{Oe} \\
)\end{array}$ & $\mathrm{K}$ & $\%$ & $\mathrm{emu} / \mathrm{g}$ & $\mathrm{K}$ & $\%$ \\
\hline $\mathrm{LP}$ & $4.7 \times 10^{-6}$ & $1.1 \times 10^{-4}$ & -1.2 & 3.1 & 1.2 & -3.5 & 2.2 \\
\hline $\mathrm{HP}$ & $1.8 \times 10^{-6}$ & $9.9 \times 10^{-5}$ & -1.6 & 2.8 & 0.9 & -1.2 & 1.7 \\
\hline $1 \%$ & $-1.5 \times 10^{-6}$ & $3.7 \times 10^{-4}$ & -0.8 & 1.0 & 2.2 & -1.9 & 1.0 \\
\hline $5 \%$ & $4.4 \times 10^{-6}$ & $1.7 \times 10^{-3}$ & -1.8 & 4.8 & 6.8 & -3.5 & 3.0 \\
\hline $10 \%$ & $3.9 \times 10^{-5}$ & $2.8 \times 10^{-3}$ & -2.5 & 8.0 & 14.9 & -3.7 & 7.0 \\
\hline
\end{tabular}




\section{Chapter 5}

\section{Phonon Dynamics in Dilute $\mathrm{CuAl}_{1-x} \mathrm{Fe}_{x} \mathrm{O}_{2}$ Delafossite Alloys by a Weighted Dynamical Matrix Approach}

\subsection{Overview:}

In this chapter, the phonon derivation from the quantum and classical views are explained. Also, the background information to understand the phonon discussion is given. The phonon dynamics by increasing the iron content is studied. Using our straightforward and computationally efficient weighted dynamical matrix (WDM) approach, we calculated the phonon dispersion in the Brillouin zone for all dilute $\mathrm{CuAl}_{1-x} \mathrm{Fe}_{x} \mathrm{O}_{2}$ alloys. Also, the WDM computational results are compared to the experimental results measured by Raman and FTIR spectroscopies. The flow chart in Figure 5.1 shows the computational and experimental steps followed to study the phonon dynamics in this chapter.

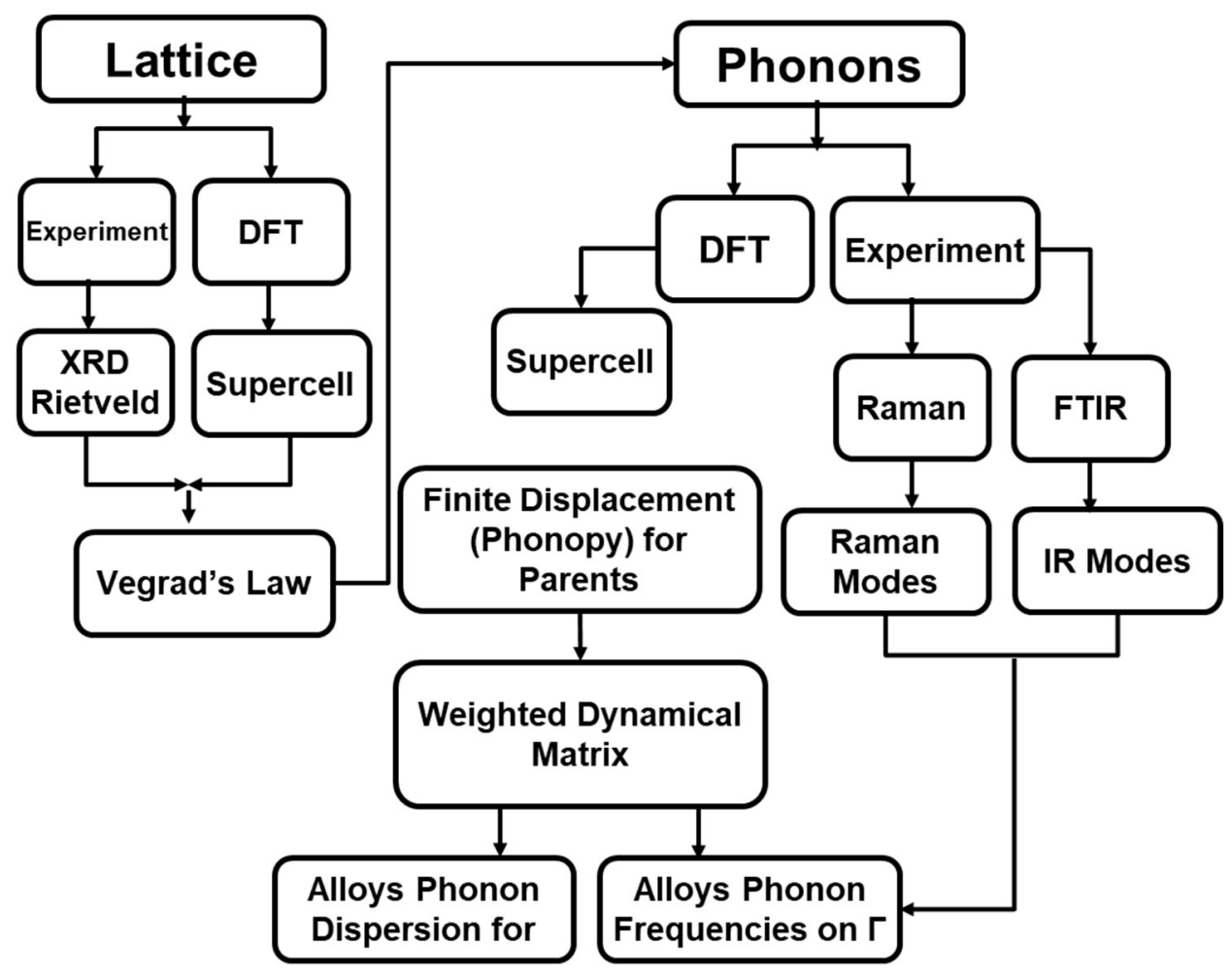

Figure 5.1: Flowchart of the phonon measurements and calculations performed on $\mathrm{CuAl}_{1-x} \mathrm{Fe}_{x} \mathrm{O}_{2}$ alloys. 


\subsection{Theory and Background Information:}

Atomic vibrations (phonons) in a periodic solid are standing elastic waves, which are the normal modes. For any crystal structure, with $N$ atoms in the primitive unit cell that are vibrating in the $3 D$ space, there should be $3 \mathrm{~N}$ phonon modes ( $\mathrm{N}$ atoms $\times 3$ dimensions $\leftrightarrow 3 \mathrm{~N}$ phonons). There are two ways to derive the phonon modes. First, from the quantum view: which considers phonons as quasi-particles. The phonon energies cab be shown as follows (elementary excitations in solids) [116]:

$E_{n}=(n+1 / 2) \hbar \omega$

The phonon quasi particle mass is $m_{0}=0$, their momentum is $p=\hbar k$, the spin is an integer $(S=0)$. Therefore, the equilibrium distribution function of phonons at temperature $\mathrm{T}$ can be found from the BoseEinstein statistics $n(\omega, T)=1 /\left[\exp \left(\hbar \omega / k_{\mathrm{B}} T\right)-1\right]$.

Second, from the classical view: the atomic bonds are assumed like an elastic spring. Therefore, the single atomic physical system can be explained by Hook's law:

$m \ddot{x}=-k x \rightarrow \omega=\sqrt{\frac{k}{m}}$

where $x$ is the displacement due to displacement of atoms from their equilibrium position, $k$ is the spring constant, and $\omega$ is the vibration frequency. We can generalize this equation of motion to the following $N$ atomic equation for a crystal structure in 3D, in a primitive unit cell [116]:

$$
\begin{aligned}
& {[\omega(\mathbf{q}, \kappa)]^{2} \chi(\mathbf{q}, \kappa)=\sum_{i \prime \kappa^{\prime}} \mathrm{D}_{i i^{\prime}, \kappa \kappa^{\prime}}(\mathbf{q}) \chi_{i \prime}(\mathbf{q}, \kappa)} \\
& \chi_{i \prime}(\mathbf{q}, \kappa)=\frac{1}{\sqrt{m_{i}}} u_{i}(\mathbf{q}, \kappa), \kappa=1,2,3 \text { and } i=1, \ldots, N \\
& \mathrm{D}_{i i^{\prime}, \kappa \kappa^{\prime}}(\mathbf{q})=\frac{1}{\sqrt{m_{i} m_{i^{\prime}}}} \Phi_{i i^{\prime}, \kappa \kappa^{\prime}}(\mathbf{q})
\end{aligned}
$$


In the above equations, $\kappa$ is the spatial dimensions, $i$ is the number of an atom in a primitive unit cell, D is the dynamical matrix, $m_{\mathrm{i}}$ is the mass of an $i$ th atom in a unit cell, $u_{\mathrm{i}}$ is the atomic displacement vector, and $\Phi$ is the second derivatives of the crystal potential. The matrix form of Equation (4) is $\omega^{2} \chi(\mathbf{q})=$ $\mathrm{D}(\mathbf{q}) \chi(\mathbf{q})$. In this equation, $\chi$ is a $3 N \times 1$-dimension matrix and $3 N \times 3 N$. By solving the eigenvalue equation (6), the values of phonon modes can be calculated.

Longitudinal Acoustic
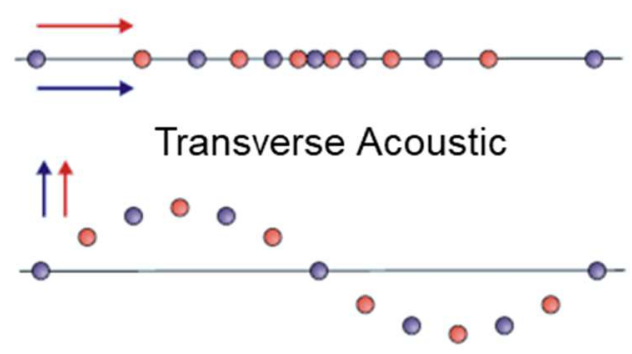

Figure 5.2: Schematic view of four possible phonon modes for a one-dimensional atomic chain.

Longitudinal Optical

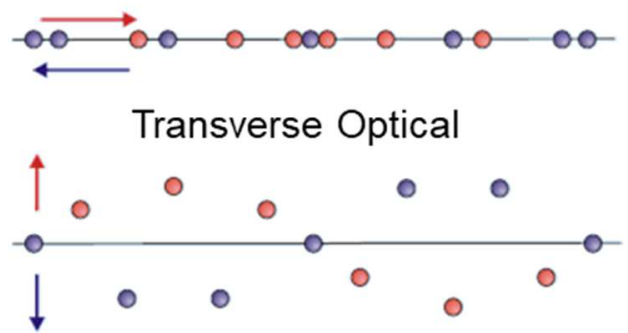

There are two types of Phonons. Acoustic phonon modes originate from in-phase displacements, and Optical phonon modes are coming from out-of-phase displacements. There are three acoustic modes in any $N$ atomic unit cell structure. There are $3 N-3$ optical phonons present in any $N$ atomic primitive unit cell. For example, in our material of study, $\mathrm{CuAlO}_{2}$ has four atoms in a primitive unit cell. Therefore, there are three acoustic modes and nine optical modes. The simple case of a chain of atoms is shown schematically in Figure 5.2. There are four phonon modes possible in a one-dimensional chain of atoms, including longitudinal and transverse acoustic (TA and LA) and longitudinal and transverse optical (TO and LO) modes. 


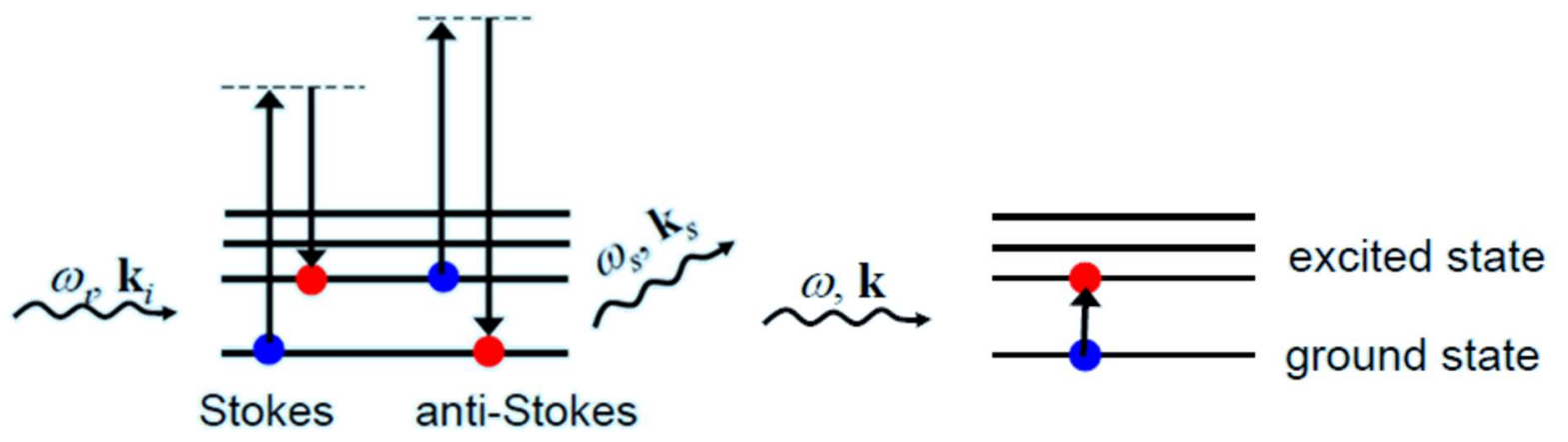

Figure 5.3: (a) Shows the Raman process schematically. (b) shows the IR absorption (FTIR) method for measurement of vibrational modes.

The types of phonon modes are dependent on the crystal symmetry, and by group theory consideration, one can find the possible modes. For more information on the determination of phonon modes by group theory, please read the references $[117,118]$.

There are various measurement techniques such as inelastic neutron scattering, X-ray spectroscopy, Fourier transform infrared spectroscopy, and Raman spectroscopy to measure the phonon frequencies. In our work, we are using Raman and FTIR spectroscopies, which provide information on the $\Gamma$-point of the Brillouin zone due to the small energy electromagnetic (EM) source used for the measurement [119-121].

The phonon modes are either Raman active or IR-active. Raman-active modes are generated from the induced dipole moment due to the deformation of the electron shell. There are Stokes and anti-Stokes modes. The Stokes modes are the frequencies that the incident frequency is smaller than the inelastically scattered EM wave. However, in anti-Stokes observation, the scarred EM wave frequency is higher than the incident EM wave [120,121]. Figure 5.3(a) shows the Raman scattering process schematically. IRActive modes are generated from the induced dipole moment due to the change in the atomic positions. This process schematically is shown in Figure 5.3(b).

\subsection{Experimental Methods:}

Room temperature Raman spectra $\left(200-2500 \mathrm{~cm}^{-1}\right)$ were acquired using a Horiba Jobin-Yvon LabRAM HR800 system (Ar ion laser, $\lambda=488 \mathrm{~nm}$, the spectral resolution of $1.5 \mathrm{~cm}^{-1}$ with a 2000 lines $/ \mathrm{mm}$ grating). The laser was focused on the powder samples with a $10 \times$ objective to obtain good signal to noise 
and auto exposed for 15 accumulations using $100 \%$ of the laser power $(50 \mathrm{~mW})$. Room temperature FTIR spectra (400-4000 $\left.\mathrm{cm}^{-1}\right)$ were acquired using a Perkin-Elmer Spectrum-Two system (spectral resolution 0.5 $\mathrm{cm}^{-1}$ ). FTIR samples were made using the $\mathrm{KBr}$ pellet method, with pellets oven-dried at $90{ }^{\circ} \mathrm{C}$ to remove adsorbed/absorbed moisture prior to acquiring the FTIR spectra.

\subsection{Phonon Dynamics:}

\subsubsection{Phonon Dispersion}

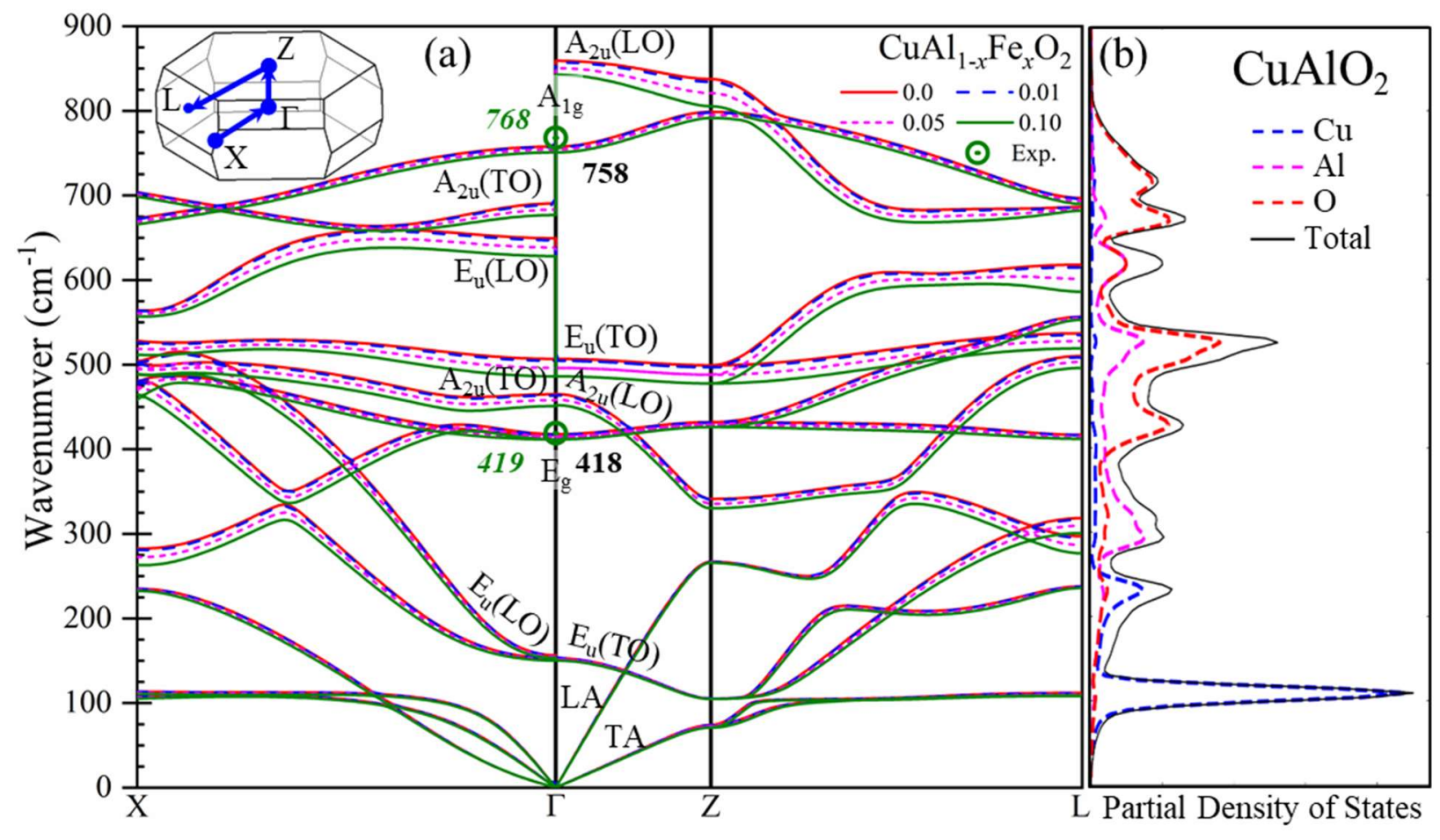

Figure 5.4: (a) shows phonon dispersion for $\mathrm{CuAl}_{1-x} \mathrm{Fe}_{x} \mathrm{O}_{2}, x=0,0.01,0.05$, and 0.10 in the path in the Brillouin zone in the inset. The experimental values of these two Raman modes for $x=0.0$ are marked as hollow circles. (b) shows the phonon density of states in the Brillouin zone

Here, we present the calculated phonon modes for $\mathrm{CuAl}_{1-x} \mathrm{Fe}_{x} \mathrm{O}_{2}(x=0,0.01,0.05$ and 0.1$)$ using weighted dynamical matrix approach. See chapter 2 for a detailed explanation of the WDM approach. Figure 5.4 (a) shows the phonon dispersion for four doping concentrations along the X-Z- $\Gamma$-L paths (Brillouin zone is shown, inset). For $\mathrm{CuAlO}_{2}$, with its four-atom primitive cell, there are twelve vibrational modes from group theory. At the $\Gamma$-point (Brillouin zone center), the modes have the symmetry: $\Gamma=\mathrm{E}_{\mathrm{g}}+$ $A_{1 g}+3 A_{2 u}+3 E_{u}$. There are three acoustic modes with $\Gamma_{\text {Acoustic }}=A_{2 u}+E_{u}$ symmetry and zero 
frequency; two Raman-active modes $\mathrm{E}_{\mathrm{g}}$ (doubly degenerate) and $\mathrm{A}_{\mathrm{lg}}$; and six IR-active modes of $\mathrm{E}_{\mathrm{u}}$ and $\mathrm{A}_{2 \mathrm{u}}$, which are optical phonons $[117,118]$. The experimental values for these two Raman modes are marked as hollow circles to compare with the experiment. In our calculations, we consider Born-effective charges by a non-analytical term added to the dynamical matrix that breaks the degeneracy in the doubly degenerate modes. In general, LO modes have a higher frequency than TO modes. By alloying $\mathrm{Fe}$ in $\mathrm{CuAlO}_{2}$, there is a clear decrease in the peak frequencies (red-shift) associated with the larger ionic radius and mass of Fe atoms, leading to a reduction of the interatomic force constants and an increase in the weighted average mass of the alloys compared to $\mathrm{CuAlO}_{2}$.

In Figure 5.4 (b), the partial density of phonon states for $\mathrm{CuAlO}_{2}$ is plotted as a function of the wavenumber over the whole Brillouin zone. $\mathrm{Cu}$ states are more noticeable at low frequencies $\left(<250 \mathrm{~cm}^{-1}\right)$. $E_{g}$ and $A_{1 g}$ Raman-active modes at $\Gamma$ are only influenced by the vibration of oxygen, as discussed in the next section, whereas the IR-active modes are the result of vibrations of all the atoms (see Figure 5.9(c)). The vibrational configurations, along with the experimental data, are presented below.

\subsubsection{Raman Spectra}

Raman spectra for the $\mathrm{CuAl}_{1-x} \mathrm{Fe}_{x} \mathrm{O}_{2}$ samples are shown in Figure 5.5. For $x=0$, there are two prominent peaks identified as $\mathrm{E}_{\mathrm{g}}$ at $419 \mathrm{~cm}^{-1}$ and $\mathrm{A}_{\mathrm{gg}}$ at $768 \mathrm{~cm}^{-1}$, in addition to some weaker peaks. These peak identifications follow the Raman studies (measurements and modeling for single-crystal $\mathrm{CuAlO}_{2}$ ) by Pellicer-Porres et al. [15]. Experimentally they see a peak at $767 \mathrm{~cm}^{-1}$ corresponding to the calculated $\mathrm{A}_{\mathrm{lg}}$ mode at $770 \mathrm{~cm}^{-1}$ involving the vibration of $\mathrm{O}$ atoms along the $c$-axis (parallel to the $\mathrm{O}-\mathrm{Cu}-\mathrm{O}$ bonds); and a peak at $418 \mathrm{~cm}^{-1}$ correspondings to the calculated $\mathrm{E}_{\mathrm{g}}$ at $433 \mathrm{~cm}^{-1}$ involving the vibration of $\mathrm{O}$ atoms perpendicular to the $c$-axis (in the hexagonal plane containing the $a$-axis). Other Raman studies in $\mathrm{CuAlO}_{2}[122-125]$ also reported the observations of these $E_{\mathrm{g}}$ and $A_{\lg }$ modes. In our powders, as doping level $x$ increases, the prominent $\mathrm{E}_{\mathrm{g}}$ and $\mathrm{A}_{\lg }$ peaks get broader and weaker and increasingly red-shifted. Also, 


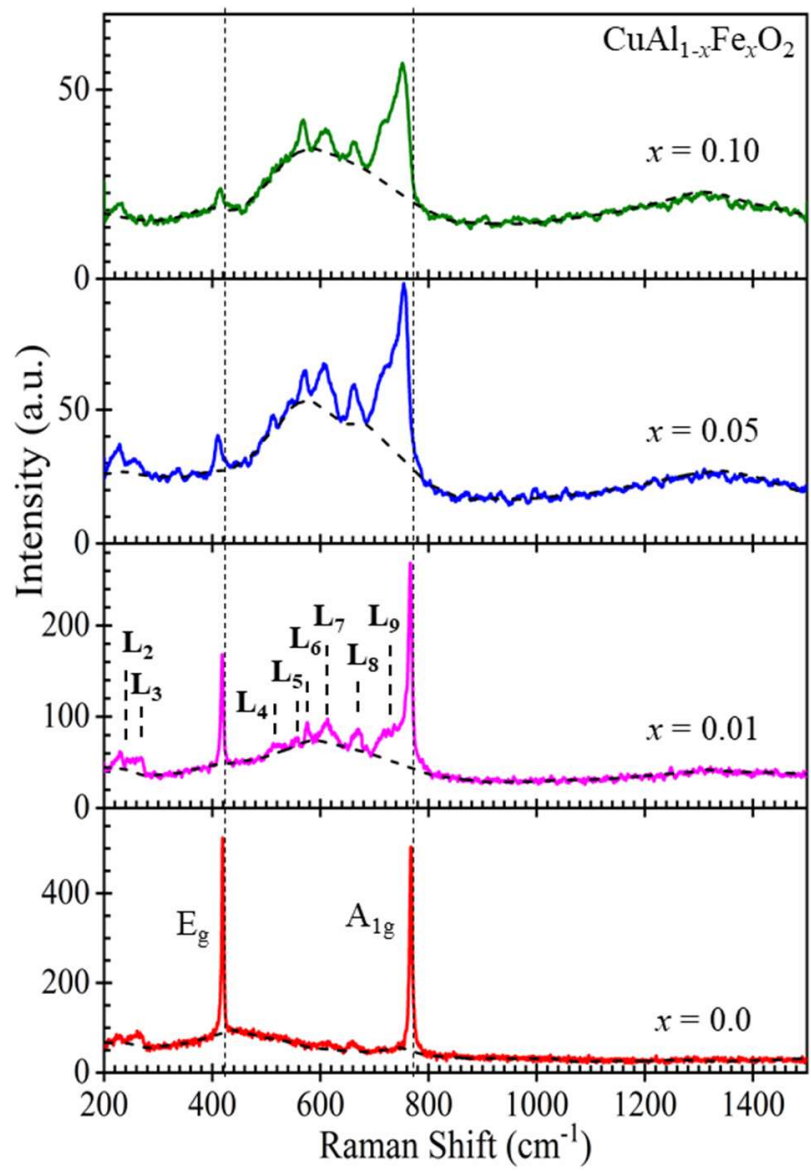

Figure 5.5: Raman spectra of $\mathrm{CuAl}_{1-x} \mathrm{Fe}_{x} \mathrm{O}_{2}, x=0,0.01,0.05$, and 0.10. $\mathrm{A}_{1 \mathrm{~g}}$ and $\mathrm{E}_{\mathrm{g}}$ modes are Raman active. Data is smoothened by averaging adjacent points. Modes change with Fe-doping due to change in $\mathrm{M}-\mathrm{O}$ bond. Dashed line shows the background. For $x=0.01, \mathrm{~L}_{2}-\mathrm{L}_{9}$ peaks (weak modes) discussed in the text are marked.

a broad background peak centered around $600 \mathrm{~cm}^{-1}$ and a second broad peak centered around 1,300 $\mathrm{cm}^{-1}$ appear in the Fe-doped samples. In addition to the $\mathrm{E}_{\mathrm{g}}$ and $\mathrm{A}_{\mathrm{lg}}$ modes, the spectra in Figure 5.5 also include several weaker peaks. These weaker peaks are similar to those reported for single crystals of $\mathrm{CuAlO}_{2}$. The peaks are associated with intrinsic defects $(\mathrm{Cu}$ vacancies and interstitial oxygen) that result in $p$-type conductivity in $\mathrm{CuAlO}_{2}$. Following the nomenclature used in [15], the observed peaks are labeled $\mathrm{L}_{2}$ through $\mathrm{L}_{9}$ in Figure 5.5. The positions of these peaks $v s . x$ are plotted in Figure 5.6. The solid symbol on the left frame show the experimental positions of the peaks for $\mathrm{CuAlO}_{2}$ reported by Pellicer-Porres et al. [15]. The $\mathrm{L}_{9}$ peak, along with the broad modes at approximately 600 and $1300 \mathrm{~cm}^{-1}$ are observed only for the Fe-doped samples and not for $\mathrm{CuAlO}_{2}$. It is likely that the broad mode at $1300 \mathrm{~cm}^{-1}$ is a second- 
order peak of the $600 \mathrm{~cm}^{-1}$ broad modes because similar second-order Raman peaks have been reported in other systems, such as graphitic samples [126].

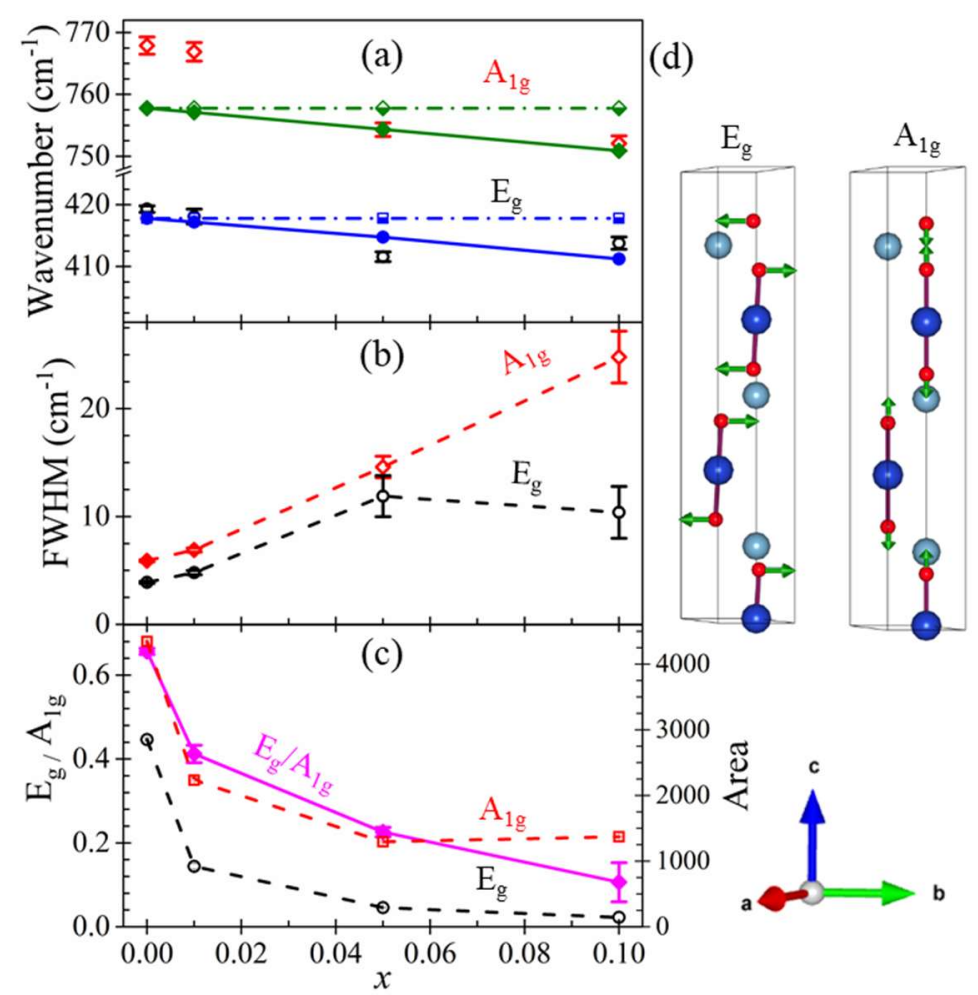

Figure 5.6: (a) Experimental peak positions of $\mathrm{E}_{\mathrm{g}}$ and $\mathrm{A}_{\mathrm{lg}}$ modes are shown as circles with error bars, compared to computed results (shown as triangular symbols with dashed lines through the points), (b) measured FWHM ) of $\mathrm{E}_{\mathrm{g}}$ and $\mathrm{A}_{1 \mathrm{~g}}$ modes, and (c) Intensity ratios (height and area under the peaks $\mathrm{E}_{\mathrm{g}} / \mathrm{A}_{1 \mathrm{~g}}$ from the Raman spectra vs. $x$ for $\mathrm{CuAl}_{1-x} \mathrm{Fe}_{x} \mathrm{O}_{2}, x=0,0.01,0.05$, and 0.10 with dashed lines as guides.

To determine the position, the full width at half maximum (FWHM), and the intensity of the $\mathrm{E}_{\mathrm{g}}$ and $A_{\lg }$ modes accurately for the Fe-doped samples, the broad peak near $600 \mathrm{~cm}^{-1}$ was subtracted out. The resulting plots of the Raman peak position, FWHM, and intensity ratios (height and area) vs. $x$ are shown in Figure 5.6 (a-c), respectively, with the schematic views of the $\mathrm{E}_{\mathrm{g}}$ and $\mathrm{A}_{\mathrm{lg}}$ Raman modes shown in Figure 5.6 (d). Figure 5.6 (a) shows a consistent red-shift for the peaks associated with the $E_{g}$ and $A_{\lg }$ modes vs. $x$; Figure 5.6 (b) shows a consistent broadening of these peaks, and Figure 5.6 (c) shows an apparent reduction in the relative strength of $E_{g}$ to $A_{1 g}$. Our discussion of these results is presented below.

\subsubsection{Red Shift of the $E_{g}$ and $A_{1 g}$ Peaks:}




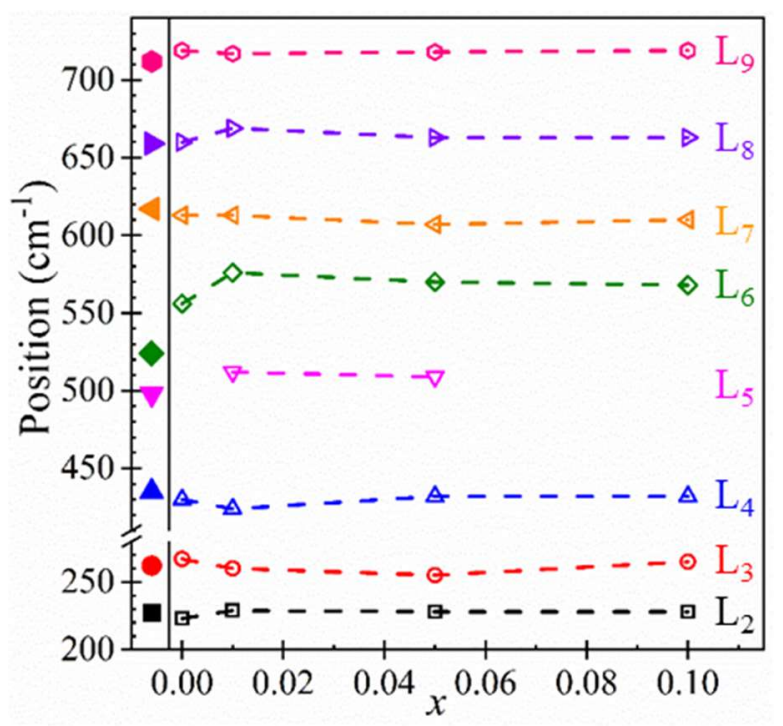

Figure 5.7: Wavenumber positions $\left(\mathrm{cm}^{-1}\right)$ of observed weak Raman modes for $\mathrm{CuAl}_{1-x} \mathrm{Fe}_{x} \mathrm{O}_{2}$ samples $(x=0,0.01$, 0.05 , and 0.1) from sample defects (open symbols). Observed (calculated) weak modes for $x=0$, from PellicerPorres 2006 Ref. [] (shown as star symbols on the left).

In Figure 5.6 (a), along with the peak positions showing the red-shift with increasing $x$, the solid lines show WDM computational results for $\mathrm{E}_{\mathrm{g}}$ and $\mathrm{A}_{\mathrm{g}}$ modes $v s . x$, and the dot-dashed (horizontal) line shows the computational results for mass-weighted average only. The Raman-active $\mathrm{E}_{\mathrm{g}}$ and $\mathrm{A}_{\mathrm{lg}}$ modes involve motion of the oxygen atoms only (see Figure 5.6 (d)), so their frequencies will not change with Fe content for a model using only mass-weighted average (prediction is horizontal). This lack of agreement with experimental results directly shows the necessity of taking into account the change in both the masses and force constants. The values and trends of calculated frequencies for our WDM approach (mass and force-constant weighted average) agree well with the experimental data. This confirms the red-shift of the optical phonon-mode associated with smaller interatomic forces and larger mass when substituting Al with $\mathrm{Fe}$, even though only the $\mathrm{O}$ is vibrating in these modes. This argument is further strengthened by directly comparing the change in properties from the $\mathrm{CuAlO}_{2}$ endpoint through to the $10 \%$ alloy to the change in properties between the $\mathrm{CuAlO}_{2}$ and endpoints (for which excellent data is available). Thus, the rate of expansion (shown in Figure 2.7 for $\left.\mathrm{CuAl}_{1-x} \mathrm{Fe}_{x} \mathrm{O}_{2}\right)$ continues until $x=1\left(\mathrm{CuFeO}_{2}\right)$. Consider this analysis for vibrational modes. Specifically, for $\mathrm{CuFeO}_{2}$, the positions of the Raman modes are $\mathrm{E}_{\mathrm{g}}=352 \mathrm{~cm}^{-1}$ and $\mathrm{A}_{\mathrm{lg}}$ $=692 \mathrm{~cm}^{-1}$ [127], representing a red-shift of the $\mathrm{E}_{\mathrm{g}}$ and $\mathrm{A}_{\mathrm{gg}}$ modes by $70 \mathrm{~cm}^{-1}(17 \%)$ and $80 \mathrm{~cm}^{-1}(10 \%)$, 
respectively, from the values for $\mathrm{CuAlO}_{2}$. The red-shift of the $\mathrm{E}_{\mathrm{g}}$ and $\mathrm{A}_{\mathrm{lg}}$ modes for $x$ up to 0.10 is $6 \mathrm{~cm}^{-1}$ $(1.4 \%)$ and $17 \mathrm{~cm}^{-1}(2.2 \%)$, respectively. This indicates the observed redshifts are the result of the expansion of the lattice constant with lengthening the $\mathrm{M}-\mathrm{O}$ bonds, which reduces their stiffness and lower these Raman frequencies. Because $\mathrm{Cu}, \mathrm{Al}$, and $\mathrm{Fe}$ atoms are stationary in the $\mathrm{E}_{\mathrm{g}}$ and $\mathrm{A}_{\mathrm{lg}}$ modes, their masses should not affect the mode frequencies. Additional support for these arguments comes from a study of the effect of particle size on the Raman spectra and lattice parameters of $\mathrm{CuAlO}_{2}$ nanoparticles down to a size of $10 \mathrm{~nm}$ [122]. The Raman modes are red-shifted by $\sim 3 \%$ with this increase in unit cell size, with $\mathrm{E}_{\mathrm{g}}$ shifting from 419 to $407 \mathrm{~cm}^{-1}$ and $A_{\text {lg }}$ from 767 to $740 \mathrm{~cm}^{-1}$ for the bulk vs. $10 \mathrm{~nm}$ particles, respectively.

\subsubsection{Broadening and Relative intensities of the $E_{g}$ and $A_{1 g}$ peaks:}

Returning to Figure 5.6 (b) shows the considerable broadening of both the $\mathrm{E}_{\mathrm{g}}$ and $\mathrm{A}_{\mathrm{lg}}$ Raman modes with increasing in $x$, and plot (c) shows the sharp decrease in the intensity ratio of the $\mathrm{E}_{\mathrm{g}} / \mathrm{A}_{\mathrm{lg}}$ modes. This is also evident in Figure 5.4, which shows that the intensity of the $\mathrm{E}_{\mathrm{g}}$ mode is reduced with an increase in $x$, to the point it is barely noticeable for $x=0.10$. The broadening of the modes with Fe doping can be related to the redshifts by the following argument. Doping $\mathrm{CuAlO}_{2}$ with Fe produces inhomogeneity in the lattice in that at sites where Fe replaces $\mathrm{Al}$, the lattice locally behaves as $\mathrm{CuFeO}_{2}$ with its Raman modes redshifted, whereas at sites without Fe doping, the Raman modes locally behave as $\mathrm{CuAlO}_{2}$. Therefore, the addition of a small amount of Fe will lead to heterogeneous broadening toward the low wavenumber side, as observed in Figure 5.5. Figure 5.6 (c) shows the behavior of the peak areas of the $E_{g}$ and $A_{1 g}$ modes and their ratio $\mathrm{E}_{\mathrm{g}} / \mathrm{A}_{\mathrm{lg}}$ with increasing $x$. Both $\mathrm{E}_{\mathrm{g}}$ and $\mathrm{A}_{\mathrm{lg}}$ peaks strongly reduce with an increase in $x$, with $\mathrm{E}_{\mathrm{g}}$ decreasing faster than $A_{1 g}$, as indicated by the decrease in the ratio $E_{g} / A_{1 g}$. These reductions are probably related to the large changes in the electronic band structure of the alloys near the excitation energy of 2.5 $\mathrm{eV}(488 \mathrm{~nm})$ even for very small $x$, and the effect of disorder associated with doping with Fe. As discussed below, for FTIR spectroscopy, the observed absorption peaks' size does not change appreciably with $x$. 


\subsubsection{FTIR Spectroscopy}

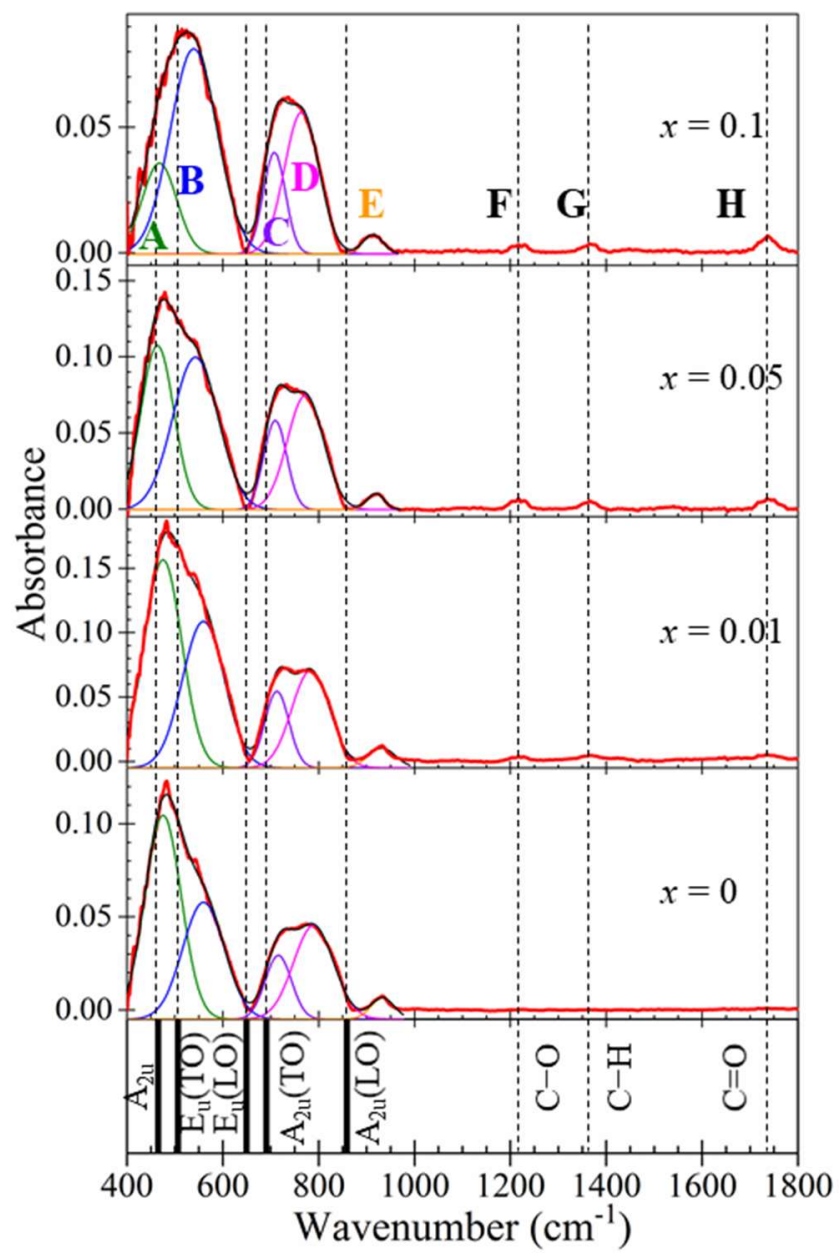

Figure 5.8: IR spectra for $\mathrm{CuAl}_{1-x} \mathrm{Fe}_{x} \mathrm{O}_{2}, x=0,0.01,0.05$, and 0.10. Peaks are labeled and the values found from the deconvolution of the peaks (see Table 5.1). F-G peaks are due to organic contaminations.

Not all the vibrational modes of a system are Raman-active. For this reason, we used FTIR spectroscopy to determine the frequencies of other $\mathrm{CuAl}_{1-x} \mathrm{Fe}_{x} \mathrm{O}_{2}$ vibrational modes to compare with our calculations. The observed FTIR absorbance spectra of the $\mathrm{CuAl}_{1-x} \mathrm{Fe}_{x} \mathrm{O}_{2}$ samples $(x=0,0.01,0.05$ and 0.10 ) in the 400 to $1800 \mathrm{~cm}^{-1}$ frequency range are shown in Figure 5.6. (For the 1800 to $4000 \mathrm{~cm}^{-1}$ range, the spectra have no distinct features.). The observed peaks are labeled as A through $\mathrm{H}$ in the top panel of Figure 6 for ease of discussion, and in the bottom panel, the sources of these peaks are identified. 

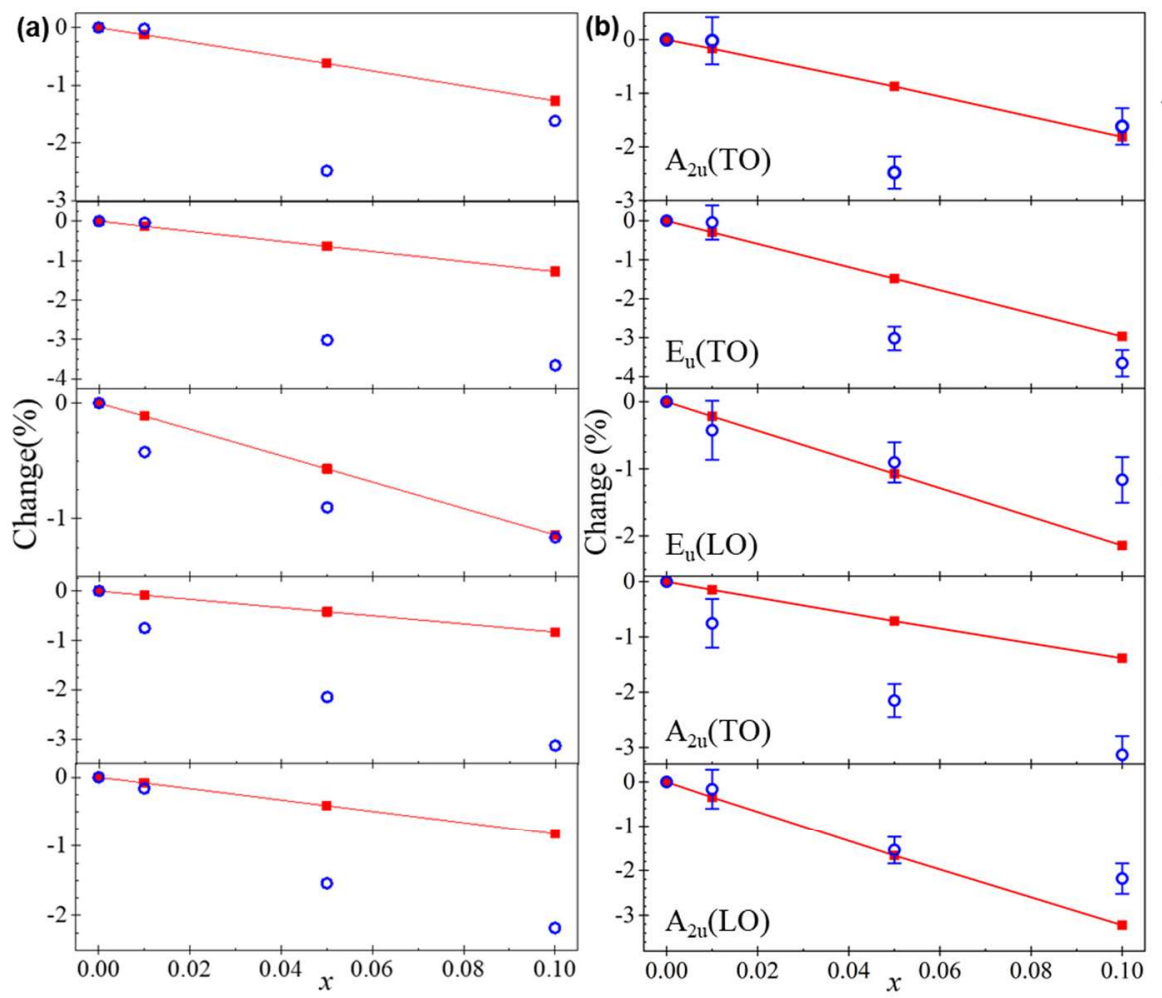

(c)
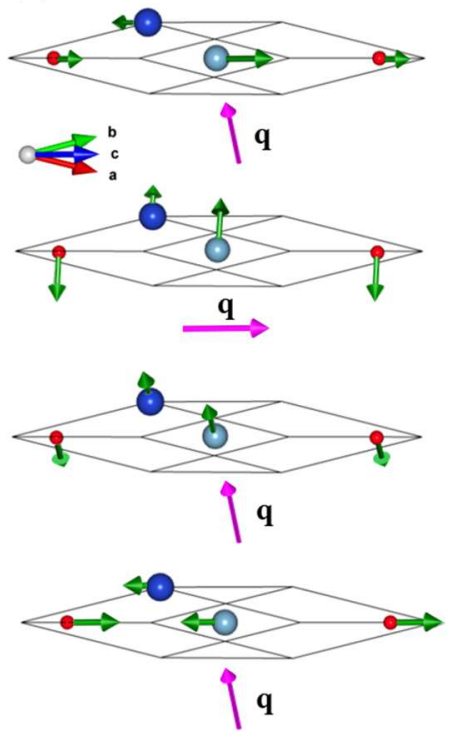

Figure 5.9: (a) Relative change in spectral line position vs. $x$ for IR-active modes: Experiment, circles and computed by renormalized mass prediction of IR modes relative change solid squares with connecting solid lines. (b) Relative change in spectral line position vs. $x$ for IR-active modes: Experiment, circles with error bars and Computed by both renormalization of mass and force constants, solid squares with connecting solid lines. (c) Schematic views of the corresponding IR vibrational modes with propagation wavevector, $\mathbf{q}$.

It is noted that the peaks $\mathrm{F}, \mathrm{G}$, and $\mathrm{H}$ are not associated with vibrational modes of $\mathrm{CuAl}_{1-x} \mathrm{Fe}_{x} \mathrm{O}_{2}$ because these peaks are independent of Fe doping and these peaks closely match the frequencies associated with the stretching modes of $\mathrm{C}-\mathrm{O}, \mathrm{C}-\mathrm{H}$, and $\mathrm{C}=\mathrm{O}$, respectively. The source of $\mathrm{F}, \mathrm{G}$, and $\mathrm{H}$ peaks are adventitious carbon layers that build upon powder samples exposed to air [128]. This agrees with our recently reported XPS studies for these powder samples, where the observed C1s peaks were identified as adventitious carbon and fitted to alkane $(\mathrm{C}-\mathrm{H})$, alcohol $(\mathrm{C}-\mathrm{OH})$, and acid-ester $(\mathrm{O}-\mathrm{C}=\mathrm{O})$ peaks [129-131]. 
The peaks labeled A to Din Figure 5.7 are de-convolved using Gaussian functions, and peaks A to E are identified according to the calculated modes shown in Figure 5.3 (a). All the peak positions and the identified mode computed using our WDM model are listed in Table 5.1. For modes A to E, the computational values are consistently smaller than our observed values, and these modes get red-shifted with an increase in $x$ (except for the A mode for $x=0.10$ ) (see table 5.1). The change in the IR-active phonon modes vs. $x$ is plotted in Figure 5.8 to facilitate the comparison between experimental and computational results. The experimental data are shown by open circles with error bars, and the computed trends are indicated by solid squares and lines for the WDM model and half-solid squares and dot-dashed line for the $A_{2 u}(L O)$ mode. The results from the WDM model agree with the experimental results within the associated error bars. The underestimation of vibrational mode wavenumbers may be related to the fact that the calculations are done for $0 \mathrm{~K}$, whereas the experimental measurements are done at room temperature. The schematic vibrational modes are shown in the primitive unit cell for each mode. All atoms are vibrating in the IR-active modes, whereas only oxygen atoms are vibrating for the Raman-active modes. From the phonon dispersion plotted in Figure 5.3 and considering the propagation wavevector direction, the modes are labeled as longitudinal optical (LO) and transverse optical (TO). The associated propagation

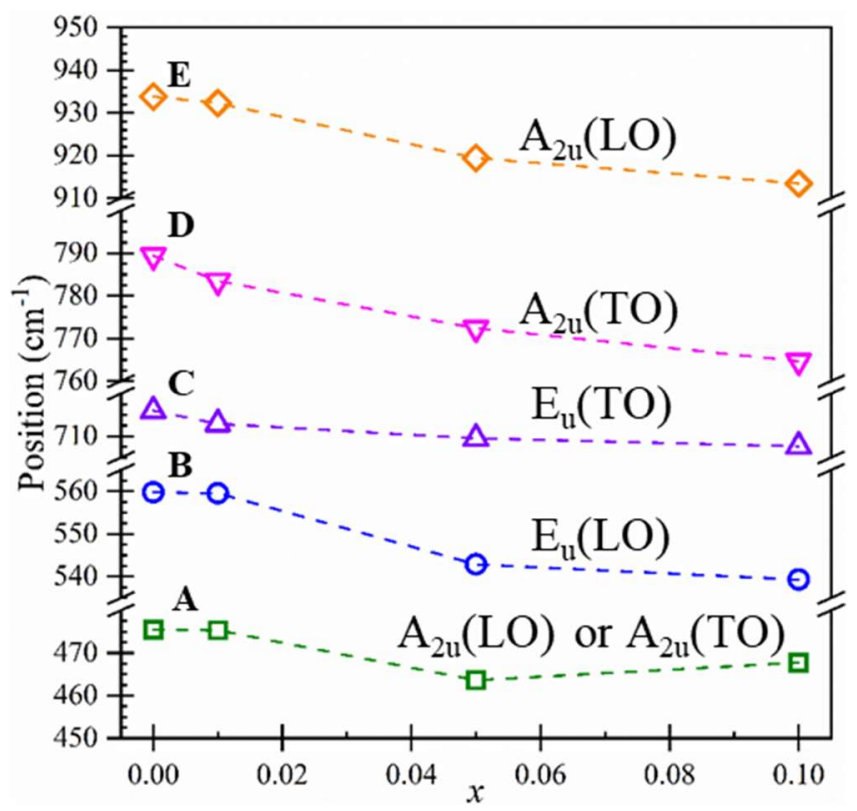

Figure 5.10: Wavenumber positions for peak positions A-E from IR spectra for $\mathrm{CuAl}_{1-x} \mathrm{Fe}_{\mathrm{x}} \mathrm{O}_{2}$, vs. $x$. Peaks are labeled as A-E (see Table 5.1). 
wavevector is shown on the schematic vibration mode representations. The $A_{2 u}(L O)$ and $A_{2 u}(T O)$ modes, both with calculated wavenumber $465 \mathrm{~cm}^{-1}$, are not resolved. Using weighted average masses only (and not the force constants) results in a change in vibration modes with $x$, as shown by the half-solid symbols connected with the dash-dotted line for the $\mathrm{E}_{\mathrm{u}}(\mathrm{TO})$ mode (second panel of Figure 5.8).

However, the agreement with our experimentally measured modes is not good, but it becomes much better when both the masses and force constants are weight averaged. The case for including the weighted average forces agrees with the findings for the Raman-active modes discussed above. This directly shows the importance of considering changes in both the force-constants and the masses. These red-shifts are qualitatively similar to those observed for the two Raman-active modes discussed earlier and are likely to have a similar origin (see Figure 5.10). Regarding the size of the absorption peaks associated with optical phonon modes (peaks A-E), qualitatively, we do not see a substantial decrease with $x$. This is in stark contrast to the large decline in peak size observed for the Raman peaks. Finally, regarding previous FTIR reports on $\mathrm{CuAl}_{1-x} \mathrm{Fe}_{x} \mathrm{O}_{2}$, we are aware of only one brief report by Lv et al. [132]. For the $x=0$ cases, they reported three modes at 513, 711, and $933 \mathrm{~cm}^{-1}$, which agree with the modes B, C, and E in Table 5.1. For the Fe-doped samples, they reported four modes, but their frequencies were not listed nor compared to the calculated values in reference [15].

\subsection{Summary and Conclusions}

In this chapter, results have been presented on the phonon modes of $\mathrm{CuAl}_{1-x} \mathrm{Fe}_{x} \mathrm{O}_{2}$ computed using several models accompanied by comparison with the Raman and FTIR modes' experimental data $\mathrm{CuAl}_{1}$ ${ }_{x} \mathrm{Fe}_{x} \mathrm{O}_{2}$ Alloy. We introduced an effective medium approach, a weighted dynamical matrix (WDM) approximation method, in the chapter and compared its results to the frequency modes measured using Raman and FTIR spectroscopy. This WDM approximation weight averages both the force constants and the alloy masses, and we directly show that this results in better agreement with the empirically measured optical phonon spectra than a more straightforward weight average of the masses only. Raman and FTIR modes for $\mathrm{CuAl}_{1-x} \mathrm{Fe}_{x} \mathrm{O}_{2}$ samples with $x=0,0.01,0.05$, and 0.10 show that the Raman-active modes, $\mathrm{E}_{\mathrm{g}}$ 
$\left(419 \mathrm{~cm}^{-1}\right), \mathrm{A}_{\lg }\left(767 \mathrm{~cm}^{-1}\right)$, and the five FTIR modes are red-shifted with an increase in $x$. The observed frequencies of these Raman and FTIR-modes agree with our calculations using the WDM approximation.

These results are consistent with the lattice expansion accompanying the Fe-doping weakening the $\mathrm{M}-\mathrm{O}$ bonds (M: $\mathrm{Al}$ or $\mathrm{Fe})$.

Table 5.1: DFT calculated and measured wavenumbers of identified IR and Raman modes for $\mathrm{CuAl}_{1-x} \mathrm{Fe}_{x} \mathrm{O}_{2}$ for $x=0$, 0.01, 0.05, and 0.10 are listed. The labels used in Figure 5.7 are shown. Peaks F-H are associated with organic contaminations.

\begin{tabular}{|l|c|c|c|c|c|c|c|c|c|c|}
\hline & & $x$ & \multicolumn{2}{|c|}{0} & \multicolumn{2}{c|}{0.01} & \multicolumn{2}{c|}{0.05} & \multicolumn{2}{c|}{0.10} \\
\hline Modes & Fig. 7 & activity & DFT & Exp. & DFT & Exp. & DFT & Exp. & DFT & Exp. \\
\hline $\mathrm{E}_{\mathrm{u}}(\mathrm{TO})$ & & IR & 153 & & 153 & & 152 & & 150 & \\
\hline $\mathrm{E}_{\mathrm{u}}(\mathrm{LO})$ & & IR & 157 & & 156 & & 155 & & 153 & \\
\hline $\mathrm{E}_{\mathrm{g}}$ & & $\mathrm{R}$ & 418 & 419 & 417 & 418 & 415 & 410 & 412 & 413 \\
\hline $\begin{array}{l}\mathrm{A}_{2 \mathrm{u}}(\mathrm{LO}) \\
\mathrm{A}_{2 \mathrm{u}}(\mathrm{TO})\end{array}$ & $\mathrm{A}$ & IR & 465 & 475 & 464 & 475 & 458 & 464 & 451 & 468 \\
\hline $\mathrm{E}_{\mathrm{u}}(\mathrm{TO})$ & $\mathrm{B}$ & IR & 507 & 560 & 505 & 560 & 496 & 543 & 486 & 539 \\
\hline $\mathrm{E}_{\mathrm{u}}(\mathrm{LO})$ & $\mathrm{C}$ & IR & 649 & 716 & 647 & 713 & 639 & 709 & 628 & 708 \\
\hline $\mathrm{A}_{2 \mathrm{u}}(\mathrm{TO})$ & $\mathrm{D}$ & IR & 691 & 789 & 689 & 783 & 683 & 772 & 677 & 765 \\
\hline $\mathrm{A}_{1 \mathrm{~g}}$ & & $\mathrm{R}$ & 758 & 768 & 757 & 766 & 754 & 754 & 751 & 751 \\
\hline $\mathrm{A}_{2 \mathrm{u}}(\mathrm{LO})$ & $\mathrm{E}$ & IR & 859 & 934 & 857 & 932 & 850 & 919 & 843 & 913 \\
\hline $\mathrm{C}-\mathrm{O}$ & $\mathrm{F}$ & IR & & 1216 & & 1217 & & 1217 & & 1217 \\
\hline $\mathrm{C}-\mathrm{H}$ & $\mathrm{G}$ & IR & & 1364 & & 1365 & & 1365 & & 1363 \\
\hline $\mathrm{C}=\mathrm{O}$ & $\mathrm{H}$ & IR & & 1735 & & 1735 & & 1735 & & 1736 \\
\hline
\end{tabular}




\section{Chapter 6}

\section{Optical Properties of Delafossite Alloys}

\subsection{Overview:}

In this chapter, the optical properties of the $\mathrm{CuAl}_{1-x} \mathrm{Fe}_{x} \mathrm{O}_{2}$ alloys are investigated experimentally and computationally. The UV-Vis-NIR spectroscopy is performed, and the electronic band structure calculations are calculated to be able to differentiate the optical defect features from the inherent optical features related to the materials. Also, in the alloys new optical absorbance feature emerges, which is also confirmed by our computational results. See the below figure that shows a summary of the chapter.

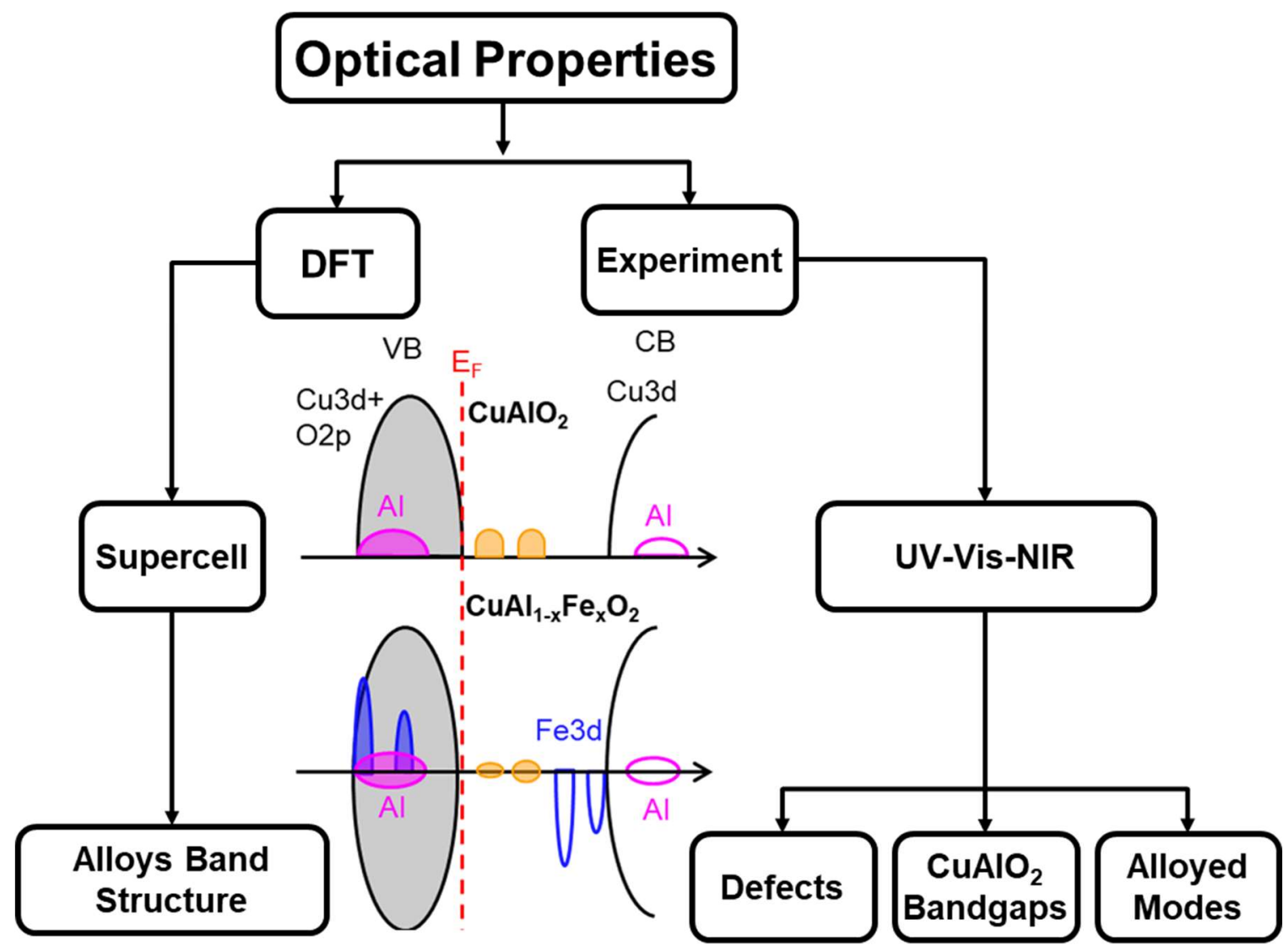

Figure 6.1: Flowchart of the experimental and computational study of the optical properties for $\mathrm{CuAl}_{1-x} \mathrm{Fe}_{x} \mathrm{O}_{2}$ alloys. 


\subsection{Theory and Background Information:}

Using ultraviolet-visible-near-infrared (UV-vis-NIR) spectroscopy, a large wavelength range of $\sim 200-2500 \mathrm{~nm}$ can be analyzed. Any wavelength of radiation that can excite electrons from the highest occupied molecular orbital (HOMO) to the lowest unoccupied molecule orbital (LUMO) gets absorbed. From the UV-Vis-NIR spectrum, the electronic transitions of the direct and indirect bandgaps, defect energy levels, present impurities, and composition information can be extracted. The UV-vis-NIR spectroscopy can be performed in both transmission and diffuse reflectance modes. The transmission mode is preferred for the transparent samples such as solid transparent thin films, solutions, solid transparent crystals, and diffuse reflectance that are appropriate for highly scattering samples such as powders and solid samples.

In the case of transmission mode, the beam of radiation, which is normal to the sample surface, goes through a sample of thickness $t$. If the incident radiation power is $P_{0}$, the power after passing through the sample is called $P$ and is calculated as follows [120]:

$$
P=P_{0} e^{-\propto t}
$$

where $\alpha$ is the absorption coefficient. The transmission coefficient is calculated as:

$T=\frac{P_{0}}{P}$

The absorbance can be calculated from Equation (6.2) with the below equation [120]:

$A=-\log T=\log \frac{P_{0}}{P}$

Whereas, in the case of diffuse reflectance mode, the scattered radiation is collected by the integrating sphere, and using the Kubelka-Munk (KM) theory, the absorption can be calculated. KM theory works best for the thick samples with more than $50 \%$ reflectance and less than $20 \%$ transmission. The particles that are composing the sample should be much smaller than the total thickness, and the sample should be evenly distributed. The KM theory is as follows [133,134]:

$F\left(R_{\infty}\right)=K / S=\left(1-R_{\infty}\right)^{2} / 2 R_{\infty}$ 
where $R_{\infty}=\underline{R}_{\text {sample }} / R_{\text {standard }}$ is the reflectance of an infinitely thick specimen, while $K$ and $S$ are the absorption and scattering coefficients, respectively.

Tauc plots are used to find the exact bandgap values from the absorption coefficient. The Tauc method is based on the assumption that the energy-dependent absorption coefficient $\alpha$ can be expressed by the following equation [135-137]:

$(\alpha \cdot \hbar \omega)^{1 / \mathrm{n}}=A\left(\hbar \omega-E_{\mathrm{g}}\right)$

where $\hbar$ is the Planck constant, $\omega$ is the photon's angular momentum, $E_{\mathrm{g}}$ is the bandgap energy, and $A$ is a proportionality constant. The $\mathrm{n}$ factor depends on the type of the electron transition and is equal to $1 / 2$ or 2 for the direct and indirect transition bandgaps $[138,139]$. If the spectrum is measured in the reflectance mode by replacing $\alpha$ with $F\left(R_{\infty}\right)$, the Tauc plot [136,140] becomes as follows:

$\left(F\left(R_{\infty}\right) \cdot \hbar \omega\right)^{1 / n}=A\left(\hbar \omega-E_{\mathrm{g}}\right)$ 


\subsection{Experimental Method:}
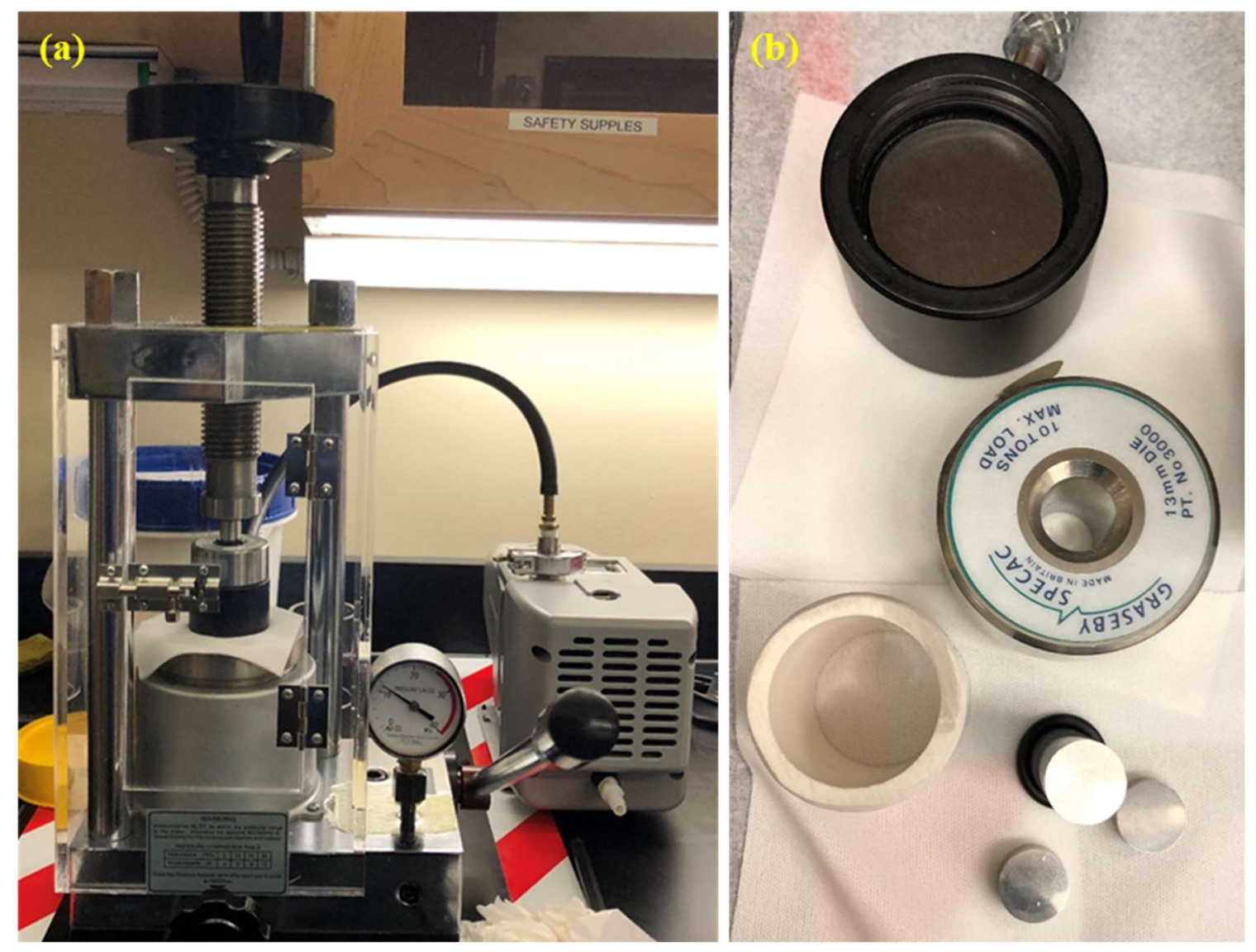

Figure 6.2: (a) Shows MTI 12 tons hydraulic press and the Agilent dry scroll pump to vacuum the die. (b) is the $1 / 2$ " die set for making our samples

The optical properties of these alloy powders in UV-visible-near IR (UV-Vis-NIR) of 1 to $6 \mathrm{eV}$ were measured at room temperature using diffuse reflectance spectroscopy employing a Shimadzu MPC3100 UV-visible spectrometer with an integrating sphere (using a $\mathrm{BaSO}_{4}$ powder reference for background correction).

PerkinElmer UV WinLab 6.4.0.971 / 2.02.06 Lambda 900 UV/VIS/NIR. We performed the optical measurements (DRS) from wavelength $250 \mathrm{~nm}$ to $2400 \mathrm{~nm}$, and the RAW file contains the wavelength and the corresponding reflectance (\%) for the four samples. However, we are unable to observe any transitions beyond $850 \mathrm{~nm}$, so all the analysis is done up-till $850 \mathrm{~nm}$.

The measurement is done using Nicolet FTIR model number Nexus 870 from Thermo Fisher Scientific. This instrument includes Ever-Glo IR and Quartz Halogen visible sources, $\mathrm{CaF}_{2}$ and $\mathrm{KBr}$ beam 
splitters, and silicone and MCTA detectors. To prepare the samples for the FTIR measurements in the transmission mode, we need to make KBr pellets. First, we dry IR grade $\mathrm{KBr}$ powder by heating on a hot plate for $24 \mathrm{~h}$ at $125 \mathrm{C}$. Otherwise, the moisture can cause the $\mathrm{KBr}$ pellet opaque. We ground the dried $\mathrm{KBr}$ powder in a mortar and pestle (sieving the powder before making the pellets can prevent from forming spots due to larger grains). We weighted up to at most $0.3 \mathrm{~g}$ of $\mathrm{KBr}$ and $0.4 \%$ sample. I will follow the same recipe. Mix the sample and $\mathrm{KBr}$ pellet very well in the mortar and pestle. We use MTI 12 tons hydraulic press to make the pellets and the die set $1 / 2$ " for making our samples, as you can see in Figure 6.2 (a) and (b). we are pulling a vacuum on the die while pressing with $3 \mathrm{Mpa}$ for 15 minutes to remove the air and help to the condensation of the pellet. Then apply $12 \mathrm{Mpa}=8$ tons for 3 minutes. After removal of the pellets, we keep the sample holder sealed by the paraffine wrapping or keeping the sample inside the vacuumed desiccators.

\subsection{Computational Method:}

The details of the density functional theory approximation simulations that are going to be discussed in the rest of this chapter are explained in Chapter 2. 


\subsection{Optical Spectroscopy:}

\subsubsection{FTIR Spectroscopy}
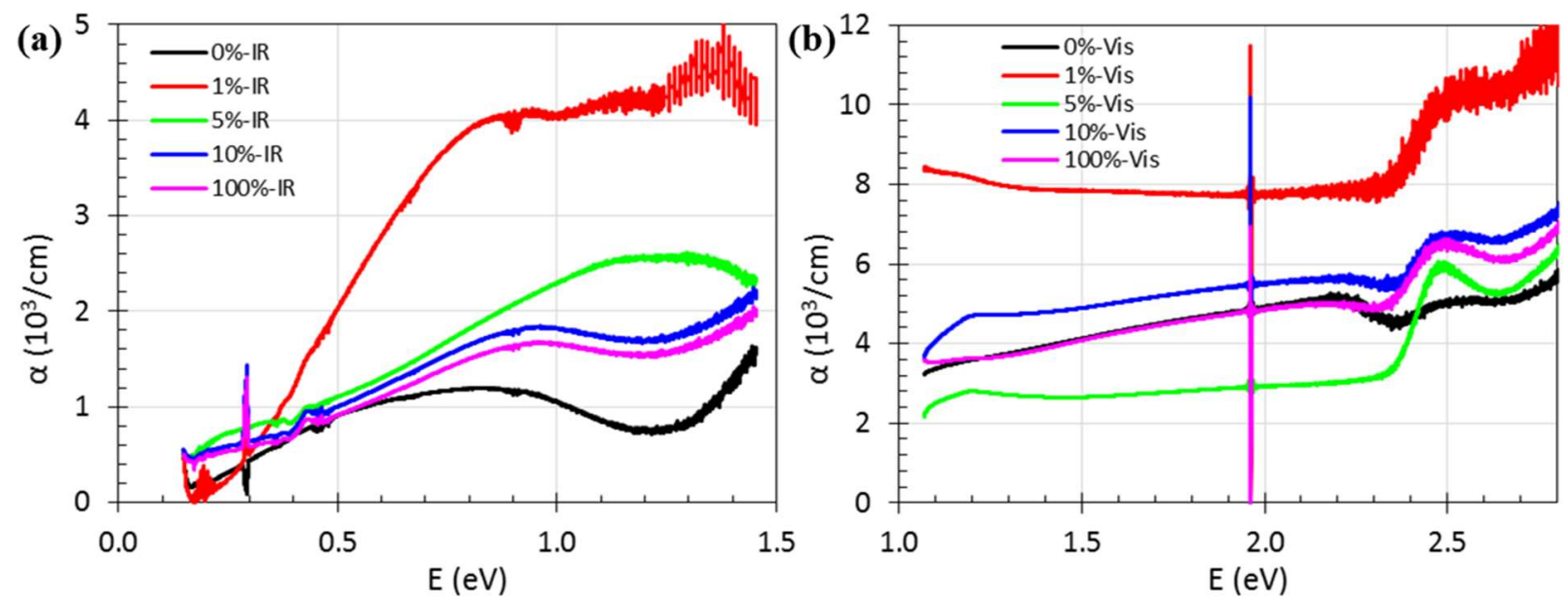

Figure 6.3: (a) and (b) Shows the absorption spectra that are measured from the $\mathrm{KBr}$ pellets in the IR and in visible range respectively for $\mathrm{CuAl}_{1-x} \mathrm{Fe}_{x} \mathrm{O}_{2}$ for $x=0.0,0.01,0.05,0.10,1.0$.

The measurement is done using Nicolet FTIR model number Nexus 870 from Thermo Fisher Scientific. This instrument includes Ever-Glo IR and Quartz Halogen visible sources, $\mathrm{CaF}_{2}$ and $\mathrm{KBr}$ beam splitters, and silicone and MCTA detectors. There are various combinations of parts that limit the measurement. We found by series of measurements on one sample that for the IR region, the best combination is the IR source, quartz beam splitter, and the MCTA detector. Unlike the visible range than a visible light source, the $\mathrm{CaF}_{2}$ beam splitter and the silicone detector work the best. The best-achieved results are shown in Figure 6.3.

Unfortunately, the results did not match well with the UV-Vis-NIR measurements, which are done in the reflection mode (explained in Section 6.5.2), probably due to a large scattering from the powder particles.

\subsubsection{UV-Vis and UV-Vis-NIR Measurements:}

Results from our computations are presented first with the $\mathrm{CuAlO}_{2}$ supercell band structure and the density of states shown in Figure 6.4 (a). The minimum of the conduction band (CB), consisting of O-2p orbitals, is located at $\Gamma$-point, and there are two local maxima in the valence band (VB), one at $L$ the other 

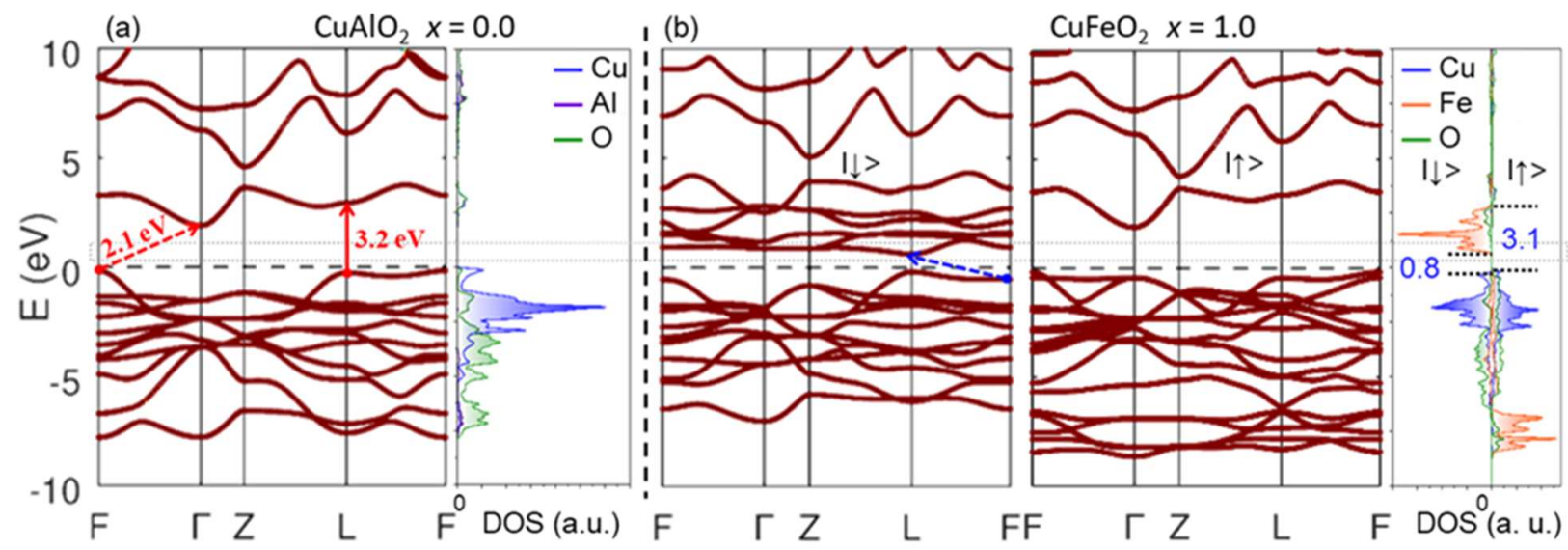

Figure 6.4: (a) Electronic band structure of $\mathrm{CuAlO}_{2}$ with the density of states, adjacent. (b) Electronic band structure of the $\mathrm{CuFeO}_{2}$ spin down $(\mid \downarrow>)$ on left spin up $(\mid \uparrow>)$ on right, as labeled, with density of states, adjacent. The black dashed lines show the fermi energy.

at $\mathrm{F}$. The minimum direct $E_{\mathrm{g}}$ of $\mathrm{CuAlO}_{2}$ is at $\mathrm{L}$, where the dipole transition between the band edge states is allowed. Vidal et al. [34] showed that all calculations (except self-consistent Green's functions) give a fundamental indirect $E_{\mathrm{g}}$ between the VB maxima at $\mathrm{F}$ and the $\mathrm{CB}$ minimum at $\Gamma$. In the literature, there is an agreement in the bandgap value of the direct transition at the L point, and it is reported in the range of 3.4-4.0 eV $[3,10-12,17]$. However, there has been a controversy over the determination of the indirect bandgap. In some reports, it is mentioned that the gap value should be $1.6-2.0 \mathrm{eV}[2,3,5]$. However, there is no observed indirect bandgap at less than $2.0 \mathrm{eV}$ in all the theoretical calculations made $[13,14,34,99]$ (apart from the results from LDA/GGA without Hubbard parameter $(U)$, which is well known for underestimating bandgaps). The indirect and direct band gaps are marked directly in Figure 6.4 (a). These gaps are expected to coincide with absorption edges in the absorption measurements described below.

The spin-polarized band structure and the density of state (DOS) of $\mathrm{CuFeO}_{2}$ are shown in Figure 6.4 (b). Because $\mathrm{CuFeO}_{2}$ is a magnetic material, the spin polarization plays a significant role in both magnetic and optical properties. The Fermi energy in the spin-up is closer to VB compared to the spindown band structure. Spin-down electronic states dominate in the range of 0.0-3.0 eV. The valence band maximum for both spin up and down consist of $\mathrm{Cu}-3 \mathrm{~d}$ bands located at the F-point. However, the 
conduction band minimum, respectively, for a spin down and up states consists of Fe-3d and O-2p, which are located at the $\mathrm{L}$ and $\Gamma$-point. The reported experimental bandgaps are the fundamental indirect bandgap

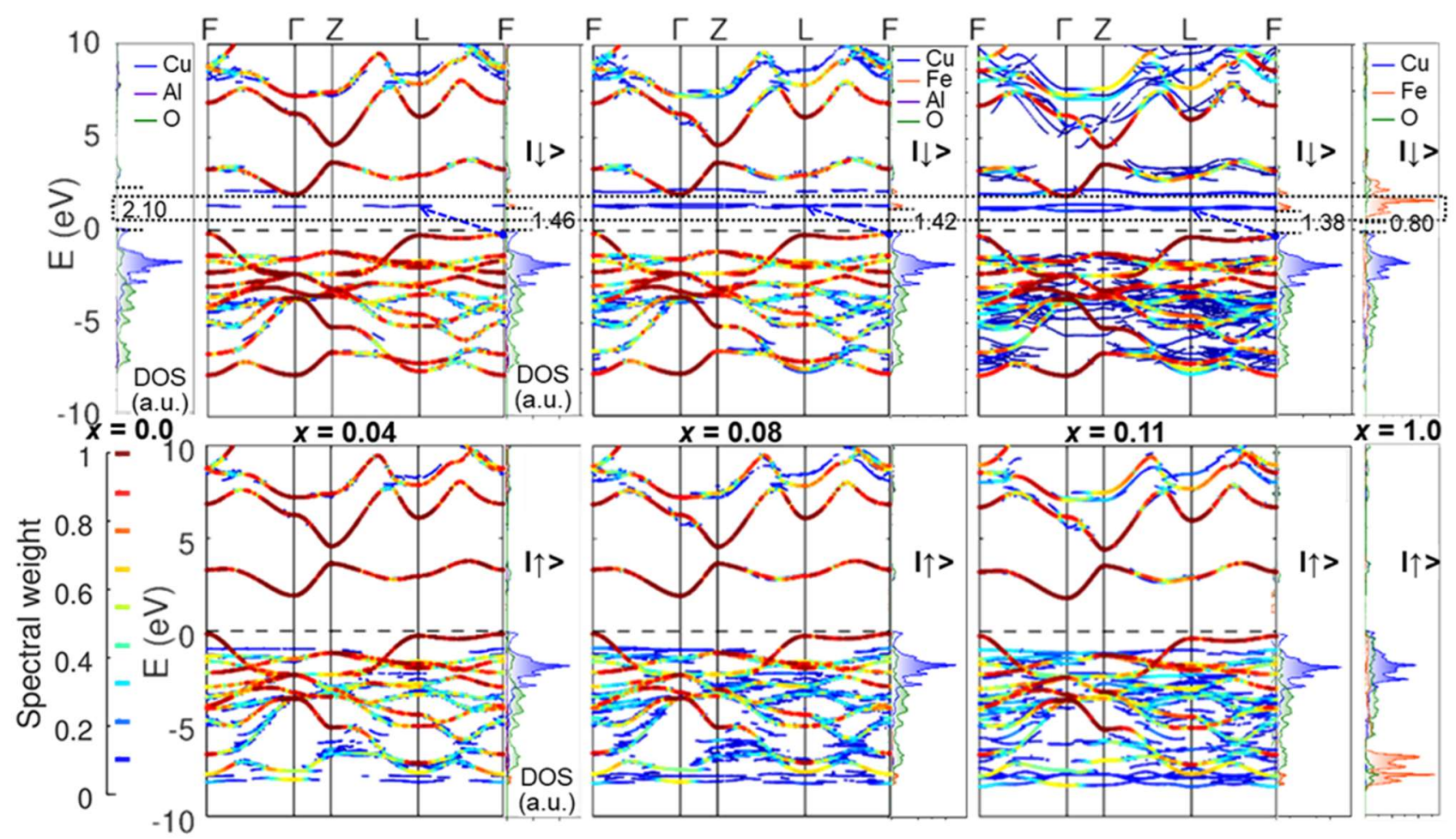

Figure 6.5: Electronic band structures of the $\mathrm{CuAl}_{1-x} \mathrm{Fe}_{x} \mathrm{O}_{2}$, calculated for $x=0.0,0.04,0.08,0.11$, and 1.0, as labeled. For $x=0.0$ and 1.0 only density of states plots is shown. For all but $x=0.0$, spin down $(\mid \downarrow>)$ is above spin up $(\mid \uparrow>)$, with spin up and spin down density of states are adjacent. The dashed lines show the fermi energy.

at $1.1 \mathrm{eV}$ and two direct bandgaps at 2.0 and $3.4 \mathrm{eV}$ [141-143]. Associating these bandgaps to the transitions in the band structure has been controversial. DOS showed in Figure 6.3 (b), and the lowest bandgap is marked for both spin up and down, respectively, 3.1 and $0.8 \mathrm{eV}$. These calculated fundamental bandgaps compare well to experimental values [141-143]. The differences in band structures of $\mathrm{CuAlO}_{2}$ and $\mathrm{CuFeO}_{2}$ are quite large, especially in the energy region of $0.8-2.1 \mathrm{eV}$ above their valence bands. $\mathrm{CuAlO}_{2}$ has no bands, whereas the Fe has introduced a rich network of bands. Optical transitions from the VB to these new Fe-related bands will mean there will be stark differences in the optical absorption spectra between $\mathrm{CuFeO}_{2}$ and $\mathrm{CuAlO}_{2}$.

The PBE $+U$ first-principle supercell calculations of the $\mathrm{CuAl}_{1-x} \mathrm{Fe}_{x} \mathrm{O}_{2}$ for $x=0.0,0.04,0.08,0.11$ and 1.0 samples are shown in Figure 6.5. The top and bottom rows show the spin-down band, and spin-up 
bands band structures, respectively, with the DOS, plotted alongside to allow the comparison of the same spin-polarizations across the figure. (The DOS is included for $x=0$ and $x=1$ without their band structures for clear comparison across the range of $\mathrm{Fe}$ concentrations). In the dilute $\mathrm{CuAl}_{1-x} \mathrm{Fe}_{x} \mathrm{O}_{2}$, two changes may be expected: (1) Due to breaking the inversion symmetry in $\mathrm{R} \overline{3} \mathrm{~m}$ structure, the forbidden direct transition at $\Gamma$-point becomes allowed. However, these band structure calculations clarify that the minimum optical transition is at the L-point, which rules out the significance of this change. (2) Spin-down Fe-3d states are added in the bandgap region of the $\mathrm{CuAlO}_{2}$ below the $\mathrm{CB}$ minimum and can decrease the fundamental bandgap. Spin-up Fe-3d orbitals are added to the VB but below VB maximum without changing the bandgap. In contrast, spin-down Fe-3d orbitals are added within the bandgap of $\mathrm{CuAlO}_{2}$, below the $\mathrm{CB}$ minimum, with a steady build-up of extent in $k$-space from $x=0.04-0.11$ [144]. Contrary to the extended states of $\mathrm{CuAlO}_{2}$, the Fe orbitals do not have a well-defined $k$, and they are only weakly present. Additionally, the upper $\mathrm{CB}$ structure of $\mathrm{CuAlO}_{2}$ and $\mathrm{CuAl}_{1-x} \mathrm{Fe}_{x} \mathrm{O}_{2}$ alloys are remarkably similar and independent of $x$. The introduction of these new Fe-related states is expected to result in optical absorption features associated with the transitions from the VB to these new energy states in these Fe-containing alloys. The constancy of the upper $\mathrm{CB}$ structure suggests the absorption features for $\mathrm{CuAlO}_{2}$ will carry over into those features in the Fe alloys investigated here. 
It is important to note that all the DFT calculations are based on single crystals with no intrinsic or extrinsic defects. However, experimentally the samples, especially powders, include impurities and defects. Furthermore, in the experimental optical absorption spectrum, there are some inevitable defect induced features. The defect absorption peaks have been identified in other studies [16,145]. However, for the powder sample, larger absorption features are observed in the recent study by Aguilar et al. [145].

Optical reflectivity spectra $(R)$ were measured at room temperature for the $\mathrm{CuAl}_{1-x} \mathrm{Fe}_{x} \mathrm{O}_{2}(x=0.0-$ 1.0) powders. The Kubelka-Munk method $[134,146]$ converts the reflectivity into the ratio of the absorption coefficient $(\alpha)$ and scattering coefficient $(S)$ by $\alpha / S=\mathrm{F}\left(R_{\infty}\right)=(1-R)^{2} / 2 R$ at any wavelength $(\lambda)$. Read a

(a)

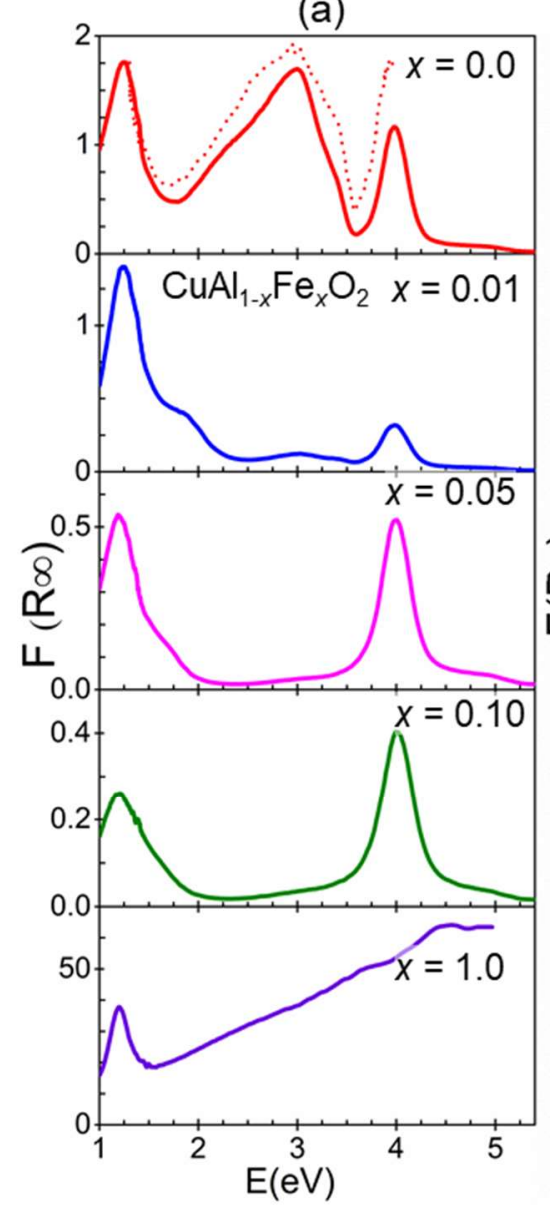

(b)

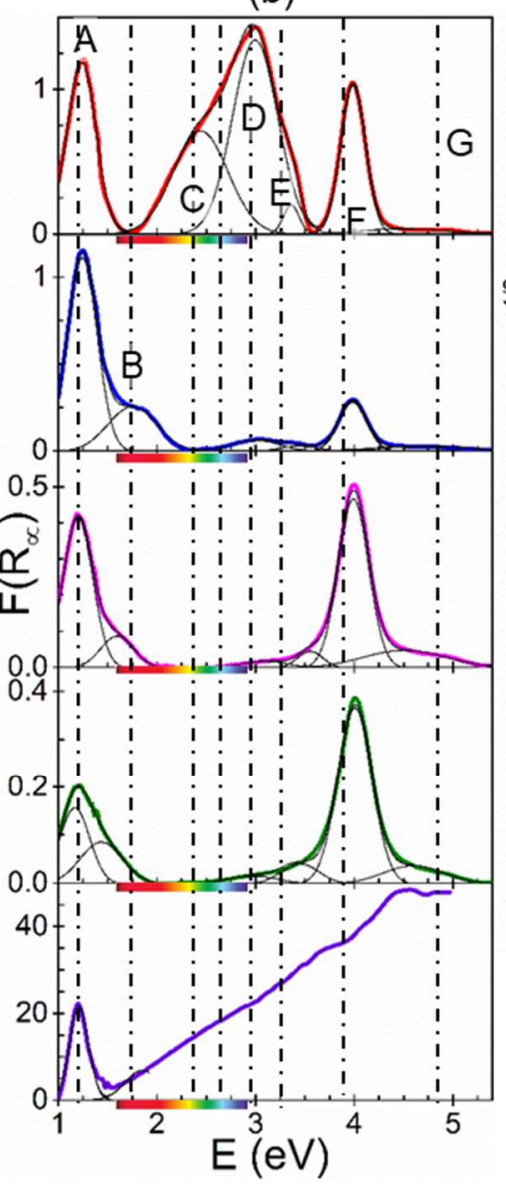

(c)

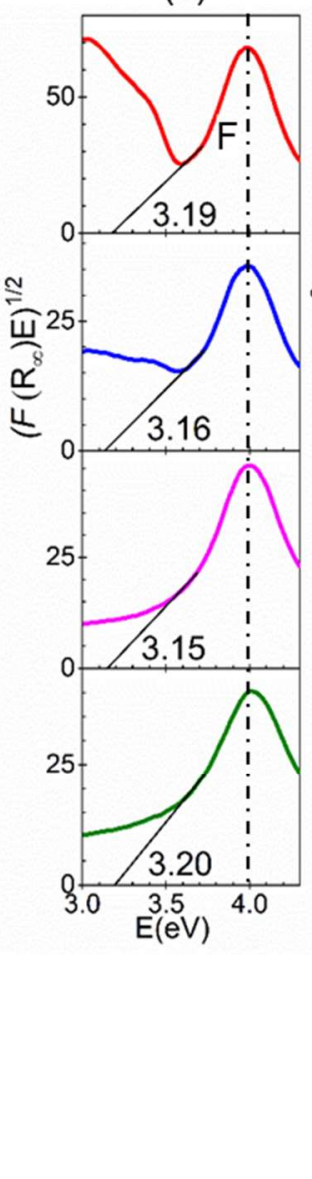

(d)

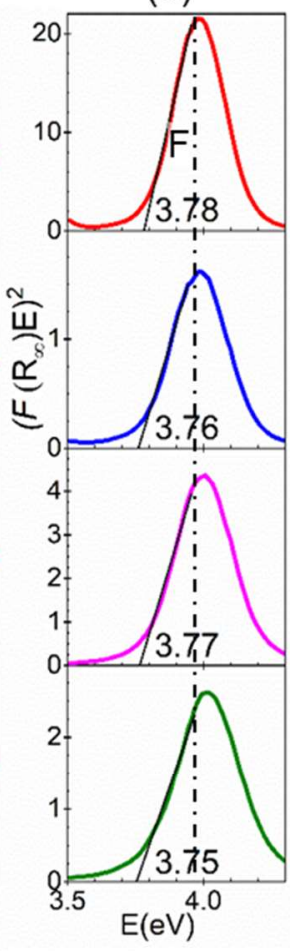

Figure 6.6: (a) $\mathrm{F}\left(\mathrm{R}_{\infty}\right)$ calculated from reflection spectra using Kobelka-Monk theory plotted for $\mathrm{CuAl}_{1-x} \mathrm{Fe}_{x} \mathrm{O}_{2}, x=$ 0.0-1.0. The dotted curve shows the spectrum presented by Aguilar et al. (b) $F\left(R_{\infty}\right)$ after background subtraction with peaks fitted using Gaussians (black solid lines and labeled A-H), (c) and (d) Tauc plots for the indirect and direct bandgaps, respectively, with the fitted lines and intercepts shown. 
detailed description in Section 6.2. Figure 6.6 (a) shows the experimentally measured $\mathrm{F}\left(R_{\infty}\right)$ for all samples along with an energy-dependent background that links the troughs between each of the observed peaks. Figure $6.6(\mathrm{~b})$ shows $\mathrm{F}\left(R_{\infty}\right)$ with the background-subtracted and deconvolution of the peaks with a series of Gaussians. In all spectra, there are multiple peaks, labeled A-G, increasing with photon energy. Peak B present for $x=0.1,0.5$, and 0.10 is clearly related to Fe content and coincides with the Fe-related feature in the band structure calculations. This peak is central to this work and is discussed below. Peak F present for $x=0.0,0.1,0.5$, and 0.10 is related to bandgaps, which the band structure calculations showed remained the same for the Fe-containing alloys we are investigating. Peak F is further investigated through the Tauc plots in Figures 6.6 (c) and (d) and discussed below. The large absorption peaks labeled A, C, and D are commonly observed for powder samples, where the surfaces have mixed oxidation states [145]. For the $\mathrm{CuAlO}_{2}$ spectra, we include absorption data for powder from a recent study [145] (dotted line) to show the agreement with our powder data. We previously showed using x-ray photoelectron spectroscopy that there is a significant amount of $\mathrm{Cu}^{2+}$ on the surface of the powder particles [8]. With that in mind, peak A at $1.2 \mathrm{eV}$ is likely due to $\mathrm{Cu}^{2+} / \mathrm{Cu}^{+}$intervalence transitions associated with mixed oxidation states [147], and peaks C, D, and E are probably due to oxygen vacancies and oxygen interstitials [16]. For completeness, the highest energy feature [148,149], peak G, is probably associated with a transition to one of the higher energy bands shown in Figures 6.4 and 6.5.

Peak $\mathrm{F}$ at approximately $4.0 \mathrm{eV}$ has been identified as the direct optical bandgap at the Lvalley $[145,150]$. Figure 6.6 (c) and (d) show Tauc plots to determine direct and indirect $E_{\mathrm{g}}$ for $x=0.0$, 0.01, 0.05, and 0.10. Please see the detailed description of the Tauc plot in Section 6.2. From the measurements, $E_{\mathrm{g}} \approx 3.8 \mathrm{eV}$ for the direct L-valley transition and $E_{\mathrm{g}}{ }^{\prime} \approx 3.2 \mathrm{eV}$ for the indirect $\mathrm{F}-\Gamma$ transition. Both values are consistent with our calculated range of optical and non-optical bandgaps. Given the proximity of peak $\mathrm{E}$ in the samples, estimates of $E_{\mathrm{g}}{ }^{\prime}$ may be less accurate than those for $E_{\mathrm{g}}$. The spectrum for $\mathrm{CuFeO}_{2}(x=1.0)$ is distinctly different from the other spectra. It shows a monotonic increase past $4 \mathrm{eV}$ with only small features. This spectrum is similar to spectra from recently measured powder $[148,149]$ 
Comparing the calculated conduction bands for $x=0.0$ and $x=1.0$ in Figure 6.4 and $x=0.01-0.10$ in Figure 6.4, it appears for $\mathrm{CuFeO}_{2}(x=1.0)$ there are many more closely spaced overlapping conduction bands (overwhelmingly spin down) from 0.8 to $2.1 \mathrm{eV}$. In absorption measurements, these conduction bands are the final states associated with absorbing electrons and will not be resolved, especially for powders, and the observed increase in absorption with increased energy follows.

Peak B is a clear indicator for Fe into the alloy. The introduction of Fe, even as little as $x=0.01$, has an evident change on $\mathrm{F}\left(R_{\infty}\right)$ spectra with the addition of peak B and the suppression of peaks C and D. Nonetheless, the defect-related peak A is observed for all samples. Additionally, the bandgap peak F also persists throughout the range of samples and maintains its spectral position. This is not entirely surprising given that even at $x=0.1$, Vegard's law predicts bandgaps closer to pure $\mathrm{CuAlO}_{2}[56]$.
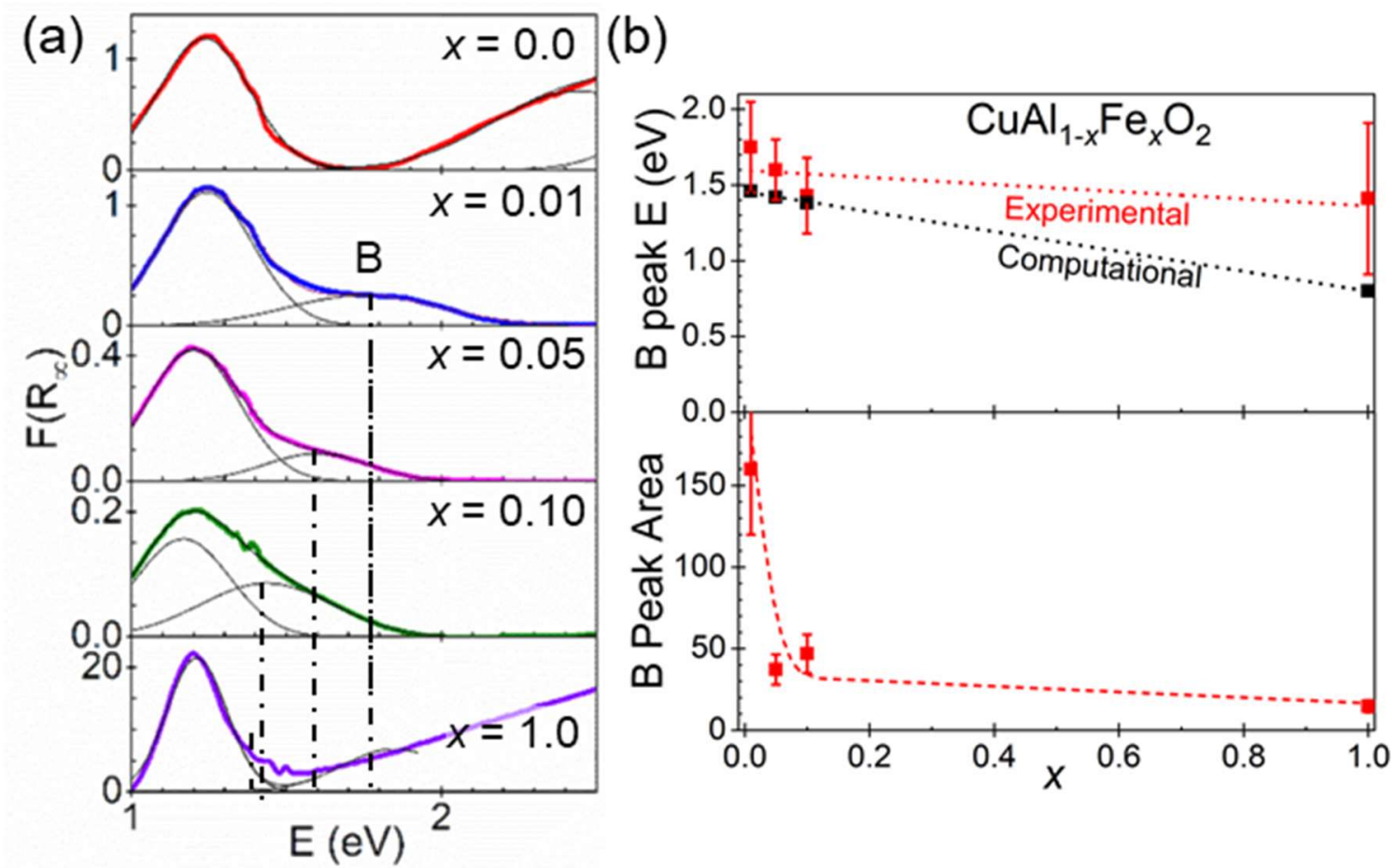

Figure 6.7: (a) Expanded view of Fe-related absorption peak (B). (b) top: Plot of the energy of the Fe-related absorption (B) and position of the Fe-related state in the modeled band structure vs. x. (b) Bottom: plot of area under the peak for the Fe-related absorption (B) and inset: plot of the area under the peak of direct bandgap peak of absorption. The dashed lines are the guide to the eye.

The position of peak B corresponds to the appearance of the Fe-3d peak in the CB of the spin-down band. Figure 6.7 (a) highlights the B peak spectra as a function of Fe concentration. Peak B is at $1.8 \mathrm{eV}$ in 
the $x=0.01$ spectrum and redshifts to $1.4 \mathrm{eV}$ by the $x=0.10$ spectrum, where it is also found in pure $\mathrm{CuFeO}_{2}$, although this position overlaps with the defect-related peak (see Figure 6.7 (b)). From the background-subtracted spectra in Figure 6.6 (b), peak B is the only peak to significantly shift with Feconcentration, which is consistent with the computed band structures. Peak B depends on (1) the introduction of $\mathrm{Fe}$ and (2) the concentration of $\mathrm{Fe}$. It is useful to directly compare the redshift in the absorbance spectra with that of the computed spin-down Fe-3d transition. This is shown in Figure 6.7 (b) (top). Both calculation and theory show the same redshift trend and reasonable quantitative agreement. The error bars are half the full width at half maximum of peak B. The experimental (squares) and computed (circles) are fitted to linear dotted lines. This is the first observation of a purely Fe-dependent mode observed in the $\mathrm{CuFe}_{x} \mathrm{Al}_{1-x} \mathrm{O}_{2}$ crystal to the best of the authors' knowledge.

Finally, in Figure 6.6 (b) (bottom), we plot the area (height times full width half maximum) of peak $\mathrm{B}$ as a function of Fe concentration. It is seen that the peak is non-existent for $\mathrm{CuAlO}_{2}$, is strong for $x=$ 0.01 , and generally decreases with increasing Fe concentration. Interestingly the peak is slightly nonmonotonic, dipping down at $x=0.05$ and slightly recovering at $x=0.10$ before continuing to decrease to its ultimate area value in $\mathrm{CuFeO}_{2}$. The dip at $x=0.05$ is reminiscent of observations in delafossite $\mathrm{CuGa}_{1}$. ${ }_{x} \mathrm{Fe}_{x} \mathrm{O}_{2}$, where $\mathrm{Fe}$ is ordered in planes as the number of $\mathrm{Fe}$ atoms is commensurate with a large supercell and produces an additional degree of long-range order [151]. Otherwise, the reduction of the area of peak B with $x$ is most likely a result of the transfer of spectral weight to the $\mathrm{F}$ peak and an addition to the overall DOS that are involved in indistinguishable interband optical transitions at photon energies above $E_{\mathrm{g}}{ }^{\prime}$. 
Furthermore, we performed UV-Vis measurements for $\mathrm{CuAl}_{1-x} \mathrm{Fe}_{x} \mathrm{O}_{2}$ for $x=0.0-1.0$ powders. The $\mathrm{F}\left(R_{\infty}\right)$ vs. energy is shown in Figure 6.8. The spectra resemble the spectra that are achieved by our Uv-VisNIR measurements. Hence, we have done a detailed analysis of the latter to avoid repetition.

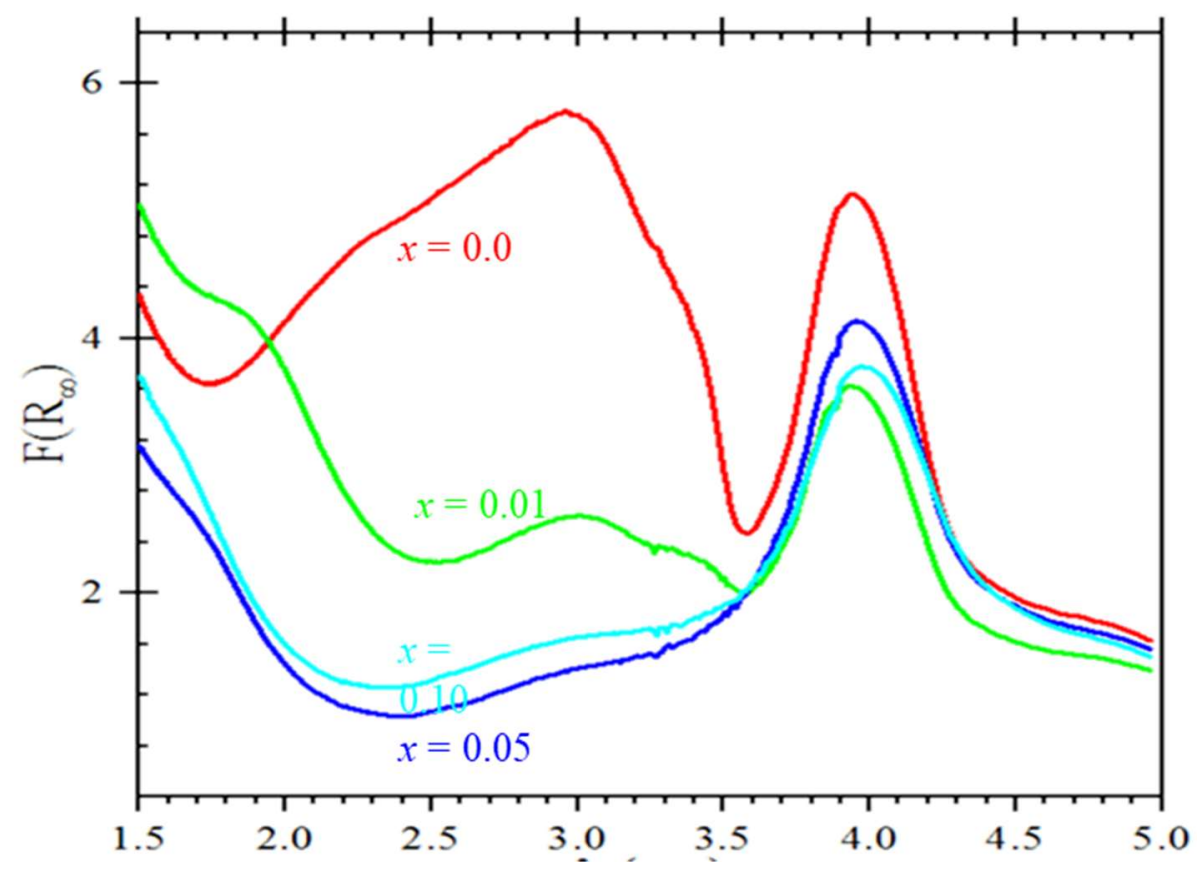

Figure 6.8: $\mathrm{F}\left(\mathrm{R}_{\infty}\right)$ calculated from reflection spectra using Kobelka-Monk theory plotted for $\mathrm{CuAl}_{1-x} \mathrm{Fe}_{x} \mathrm{O}_{2}, x=0.0$ 1.0. The spectra are similar to Figure 6.5.

\subsection{Conclusions}

The optical reflection spectra are measured for $\mathrm{CuAl}_{1-x} \mathrm{Fe}_{x} \mathrm{O}_{2}$ for $x=0.0-1.0$ powders, and band structures are calculated by the first-principle DFT. A PBE $+U$ approach is used for the calculations, and $U$ is optimized to compensate for the exciton and polaronic effects. According to the band structure calculations, the fundamental indirect bandgap is $\mathrm{F}-\Gamma$ transition, experimentally observed at $\sim 3.2 \mathrm{eV}$, and the direct optical bandgap is at the L-point, observed at $\sim 3.8 \mathrm{eV}$. The positions of these bandgaps are insensitive to Fe content $(x=0.01-0.10)$ in agreement with the computed band structures.

Fe concentration does result in a new optical feature near $\sim 1.4 \mathrm{eV}$ that undergoes a redshift with increasing concentration and is associated with Fe-3d energy levels that form at lower energy than $\mathrm{CuAlO}_{2}$ conduction band edge. Earlier work suggested alloying breaks the inversion symmetry in $\mathrm{R} \overline{3} \mathrm{~m}$ structure, 
such that the otherwise forbidden direct transition at $\Gamma$-point becomes allowed. However, the presented DFT calculations confirm that the minimum direct bandgap remains at L-point, ruling out that possibility. Nonetheless, the Fe-concentration-dependent optical feature can be tuned by composition and offers a new absorption mechanism near $1.6 \mathrm{eV}$ in $\mathrm{CuAl}_{1-x} \mathrm{Fe}_{x} \mathrm{O}_{2}$. This result makes powders of dilute-Fe delafossite alloys more appealing for applications such as photocatalytic activity. 


\section{Chapter 7}

\section{Conclusion, Future Direction, and Publications}

\subsection{Summary and Conclusion:}

The objectives of the presented research in this thesis were to investigate the effect of Fe alloying in the magnetic, optical, and vibrational properties and find out the applicability of the alloys in optoelectronics such as transparent conductive oxide photocatalyst, etc. and spintronics room temperature transparent ferromagnet applications. To synthesize high purity $\mathrm{CuAl}_{1-x} \mathrm{Fe}_{x} \mathrm{O}_{2}$ alloys using solid-state reaction and investigated the magnetic, vibrational, and optical properties computationally and experimentally.

Various methods are used to characterize the powders. The samples are characterized using the $\mathrm{XRD}$, and it is confirmed that all samples have a rhombohedral structure. By Rietveld refinement, the optimized baking time, the lattice parameters, and crystallite size are determined. Using EDS, the elemental concentration of the samples is measured, which matches well with the nominal value of the Fe concentration in all samples. Using XPS, we could determine the $\mathrm{Cu}^{+} / \mathrm{Cu}^{2+}$ ratio at the surface of the sample crystallites, which is significant. The $\mathrm{Cu}^{2+}$ is a magnetic ion and can produce a background in our magnetic measurements.

In Chapter 4, the data of the temperature and magnetic field dependence of the magnetization in the $\mathrm{CuAl}_{1-x} \mathrm{Fe}_{x} \mathrm{O}_{2}$ samples with $x=0.0,0.01,0.05$, and 0.1 are presented along with the analysis and discussion of the results. The results show that $\mathrm{Fe}$ is present as $\mathrm{Fe}^{3+}$ replacing $\mathrm{Al}^{3+}$ and the estimated value of $x$ using magnetic measurements is in good agreement with the nominal values of $x$. These results also show the absence of a magnetic phase transition in the Fe-doped samples at least down to $2 \mathrm{~K}$ and that $\mathrm{Fe}^{3+}$ $\mathrm{Fe}^{3+}$ exchange-coupling is antiferromagnetic. Because these results do not agree with the theoretical prediction of the ferromagnetic $\mathrm{Fe}^{3+}-\mathrm{Fe}^{3+}$ exchange coupling and the possible presence of a Curie temperature increasing with an increase in $x$ [53], a revised look at the relevant theoretical analysis [53] is warranted. The hysteresis loops and weak ferromagnetism observed at $300 \mathrm{~K}$ for the Fe-doped samples are 
shown to be likely due to the presence of hematite as an impurity in the samples. This impurity possible results from some un-reacted hematite, one of the precursors used in the solid-state synthesis of the $\mathrm{CuAl}_{1}$ ${ }_{x} \mathrm{Fe}_{x} \mathrm{O}_{2}$ samples. Since the small level of this impurity is not detected in the standard XRD analysis of the samples, magnetic measurements, as reported here, are essential to detect this impurity. Because hematite is a weak ferromagnet at room temperature, RTFM presence in the impurity-free samples of $\mathrm{CuAl}_{1-x} \mathrm{Fe}_{x} \mathrm{O}_{2}$ samples is highly unlikely.

In Chapter 5, results have been presented on the phonon modes of $\mathrm{CuAl}_{1-x} \mathrm{Fe}_{x} \mathrm{O}_{2}$ computed using several models accompanied by comparison with the experimental data on the Raman and FTIR modes in powder samples of $\mathrm{CuAl}_{1-x} \mathrm{Fe}_{x} \mathrm{O}_{2}$. Using a disordered supercell, we model the structure of the lattice and find good agreement with our Rietveld- refined XRD results. For the calculation of lattice dynamics, continuing with this disordered supercell is computationally too demanding. Instead, we introduce a practical medium approach, a weighted dynamical matrix (WDM) approximation method, and compare its results to the frequency modes measured using Raman and FTIR spectroscopy. This WDM approximation weight averages both the force constants and the alloy masses. We directly show that this results in better agreement with the empirically measured optical phonon spectra than a more straightforward weight average of the masses only. Raman and FTIR modes for $\mathrm{CuAl}_{1-x} \mathrm{Fe}_{x} \mathrm{O}_{2}$ samples with $x=0,0.01,0.05$, and 0.10 show that the Raman-active modes, $E_{\mathrm{g}}\left(419 \mathrm{~cm}^{-1}\right) \mathrm{A}_{\mathrm{lg}}\left(767 \mathrm{~cm}^{-1}\right)$, and the five FTIR modes are redshifted with an increase in $x$. The observed frequencies of these Raman and FTIR-modes agree with our calculations using the WDM approximation. These results are consistent with the lattice expansion accompanying the Fe-doping weakening the $\mathrm{M}-\mathrm{O}$ bonds ( $\mathrm{M}$ : $\mathrm{Al}$ or $\mathrm{Fe})$.

In Chapter 6, the optical reflection spectra are measured for $\mathrm{CuAl}_{1-x} \mathrm{Fe}_{x} \mathrm{O}_{2}$ for $x=0.0-1.0$ powders, and the first-principle DFT calculates band structures. A PBE $+U$ approach is used for the calculations. According to the band structure calculations, the fundamental indirect bandgap is $\mathrm{F}-\Gamma$ transition, experimentally observed at $\sim 3.2 \mathrm{eV}$, and the direct optical bandgap is at the L-point, observed at $\sim 3.8 \mathrm{eV}$. The positions of these bandgaps are insensitive to Fe content $(x=0.01-0.10)$ in agreement with the computed band structures. Fe concentration does result in a new optical feature near $\sim 1.4 \mathrm{eV}$ that undergoes 
a redshift with increasing concentration and is associated with Fe-3d energy levels that form at lower energy than $\mathrm{CuAlO}_{2}$ conduction band edge. Earlier work suggested alloying breaks the inversion symmetry in $\mathrm{R} \overline{3} \mathrm{~m}$ structure, such that the otherwise forbidden direct transition at $\Gamma$-point becomes allowed. However, the presented DFT calculations confirm that the minimum direct bandgap remains at L-point, ruling out that possibility. Nonetheless, the Fe-concentration-dependent optical feature can be tuned by composition and offers a new absorption mechanism near $1.6 \mathrm{eV}$ in $\mathrm{CuAl}_{1-x} \mathrm{Fe}_{x} \mathrm{O}_{2}$. This result makes powders of dilute-Fe delafossite alloys more appealing for applications such as photocatalytic activity.

\subsection{Future Direction:}

Also, delafossite alloys are a less studied group of oxides that are diverse in their properties and possible applications. In this thesis, I studied the $\mathrm{CuAl}_{1-x} \mathrm{Fe}_{x} \mathrm{O}_{2}$ alloys. There are various aspects of this thesis to be improved in the future. Also, this work can be the road map for the investigation of other alloys. In the following paragraphs, I describe the work that is in progress and the other future work that can be done.

One of the challenges of this work was working on powder samples. As the powders have defects and high optical scattering, the optical measurements are harder to perform. To improve the samples' quality, they should be grown as thin films on transparent substrates such as c-plane sapphire.

I investigated the $\mathrm{CuAl}_{1-x} \mathrm{Fe}_{x} \mathrm{O}_{2}$ alloys up to $10 \%$, and I could not see any magnetic ordering for the temperature between 2 to $300 \mathrm{~K}$ and fields up to $90 \mathrm{kOe}$. However, we did not observe any Phase transition or critical temperature, such as Neel temperature of Curie temperature. The study of the magnetic properties of $\mathrm{CuAl}_{1-x} \mathrm{Fe}_{x} \mathrm{O}_{2}$ alloys needs to be performed at lower than $2 \mathrm{~K}$ temperatures and higher magnetic fields for achieving more accurate Fe concentration estimation using modified Brillouin function. Also, we need to investigate the possibility of a phase transition temperature at lower than $2 \mathrm{~K}$ temperature. Furthermore, we can make high purity powders with a higher concentration of Fe. Moreover, the computational 
investigation of the alloys done by Kizaki et al. did not match with the experimental results. Hence, the calculations should be troubleshot and probably performed again.

We have studied the optical properties of the alloys thoroughly computationally using the electronic band structure calculations and experimentally and confirmed a new optical mode that can change the absorption in the optical range. The photocatalysis measurements are necessary to confirm how alloying can, in practice, impact the photocatalysis. Also, some optical measurements such as the time-resolved ultrafast measurement can help to understand the nature of red-shift in the new optical mode by alloying. There is a gap in the optical investigation of $\mathrm{CuAl}_{1-x} \mathrm{Fe}_{x} \mathrm{O}_{2}$ alloys by the terahertz and ultrafast methods, which can provide useful information about the alloys' photoconductivity. Further optical measurements can be helpful in understanding the photocatalytic process by providing information about the dynamics of the charge carriers.

We performed the phonon calculations for the dilute $\mathrm{CuAl}_{1-x} \mathrm{Fe}_{x} \mathrm{O}_{2}$ alloys using our proposed weighted dynamical matrix approach (WDM), which agreed well with our experimental results. However, the versatility of this approach and the pros/cons are not widely tested. We need to test this approach on several alloys such as binary, ternary, and high entropy random alloys and compare the results with the available computational and experimental results reported in the literature. We need to prepare the programs written by us as a package and make it available on GitHub for the users interested in using this research approach.

Finally, there are various transparent delafossite choices with similar properties to $\mathrm{CuAlO}_{2}$, such as $\mathrm{CuCrO}_{2}, \mathrm{CuInO}_{2}, \mathrm{CuSrO}_{2}$, etc. A similar exploration can be performed on their alloys for their possible applications in spintronics, photocatalysis, and optoelectronics.

\subsection{Publications of the Author Related to this Dissertation:}

Descriptions and discussion of the results presented in this dissertation have appeared in literature with details given below. 
- M. Aziziha; S. Akbarshahi; S. Ghosh; S. Pittala; R. Sooriyagoda; J.P. Lewis; A.H. Romero; S. Thota; A.D. Bristow; M.S. Seehra; and M.B. Johnson,” Identification of a Fe-concentrationDependent Optical Mode in $\mathrm{CuAl}_{1-x} \mathrm{Fe}_{x} \mathrm{O}_{2}$ " (Under review)

- M. Aziziha, S. Akbarshahi, S. Ghosh, P. Pramanik, R. Sooriyagoda, A. Romero, S. Thota, S. Pittala, A. Bristow, M. Seehra, and M. Johnson, "Optical Properties of Dilute CuAl1-xFexO2 Delafossite Alloys," in Frontiers in Optics / Laser Science, (Optical Society of America, 2020), paper $\underline{J M 6 A .31} .[54]$

- M. Aziziha; S. Akbarshahi; S. Ghosh; P. Pramanik; J.P. Lewis; A.H. Romero; S. Thota; M.S. Seehra; and M.B. Johnson, "Phonon Dynamics in Anisotropic Dilute $\mathrm{CuAl}_{1-x} \mathrm{Fe}_{x} \mathrm{O}_{2}$ Delafossite Alloys by a Weighted Dynamical Matrix Approach", J. Phys. Chem. C 123, 50, 30604-30612 $\underline{(2019)} \cdot[48]$

- M. Aziziha; R. Beesley; S.A. Byard; J.P. Lewis; M.S. Seehra; and M.B. Johnson, "Magnetic Properties of Fe-doped $\mathrm{CuAlO}_{2}$ and Role of Impurities", AIP Advances 9, 035030 (2019). [8]

- $\quad$ M. Aziziha; R. Beesley; J. Magers; N. Mottaghi; M.B. Holcomb; J.P. Lewis; M.S. Seehra; and M.B. Johnson, "Electronic state and concentration of $\mathrm{Fe}^{3+}$ in $\mathrm{CuAl}_{1-x} \mathrm{Fe}_{x} \mathrm{O}_{2}$ determined by magnetic measurements", J. Magn. Magn. Mater. 471, 495 (2019). [7]

\subsection{Other Publications of the Author:}

- $\quad$ M. Ahmed; M. Aziziha; M.B. Johnson; L.S. Lin, "Magnetic sludge by-products for adsorptive removal of phosphorus: resource recovery from iron-based anaerobic biological wastewater treatment" (Accepted)

- D. Schrecongost; M. Aziziha; H. Zhang; I.-C. Tung; J. Tessmer; W. Dai; Q. Wang; R. Engel-Herbert; H. Wen; Y.N. Picard; and C. Cen, "On-Demand Nanoscale Manipulations of Correlated Oxide Phases", Adv. Funct. Mater. 2019, 190558 (2019). [152] 
- $\quad$ K.V.I. Sivaneri; O. Ozmen; M. Aziziha; E.M. Sabolsky; T.H. Evans; D.B. DeVallance; and M.B. Johnson, "Robust polymer-HfO 2 Thin Film Laminar Composites for Tactile Sensing Applications", Smart Mater. Struct. 28, 025002 (2019). [138]

- $\quad$ N. Mottaghi; R.B. Trappen; S. Kumari; C.-Y. Huang; S. Yousefi; G.B. Cabrera; M. Aziziha; A. Haertter; M.B. Johnson; M.S. Seehra; and M.B. Holcomb, "Observation and interpretation of negative remanent magnetization and inverted hysteresis loops in a thin film of $\mathrm{La}_{0.7} \mathrm{Sr}_{0.3} \mathrm{MnO}_{3}{ }^{\prime}, \underline{J}$ Phys.: Condens. Mater 30, 405804 (2018). [113] 


\section{References:}

[1] J. L. Lyons, A. Janotti, and C. G. Van de Walle, Semiconductors and Semimetals, Vol. 88 (2013).

[2] H. Kawazoe, M. Yasukawa, H. Hyodo, M. Kurita, H. Yanagi, and H. Hosono, P-Type Electrical Conduction in Transparent Thin Films of CuAlO2, Nature 389, 939 (1997).

[3] H. Yanagi, S. Inoue, K. Ueda, H. Kawazoe, H. Hosono, and N. Hamada, Electronic Structure and Optoelectronic Properties of Transparent P-Type Conducting CuAlO2, Journal of Applied Physics 88, 4159 (2000).

[4] X. Nie, S.-H. Wei, and S. B. Zhang, Bipolar Doping and Band-Gap Anomalies in Delafossite Transparent Conductive Oxides, Physical Review Letters 88, (2002).

[5] F. A. Benko and F. P. Koffyberg, Opto-Electronic Properties of CuAlO, Journal of Physics and Chemistry of Solids 45, 57 (1984).

[6] M. Kumar, H. Zhao, and C. Persson, Study of Band-Structure, Optical Properties and Native Defects in $\mathrm{A}^{I} \mathrm{~B}^{I I I} \mathrm{O}_{2}$ ( $\mathrm{A}^{I}=C u$ or $\mathrm{Ag}$, B ${ }^{I I I}=\mathrm{Al}$, Ga or In) Delafossites, Semiconductor Science and Technology 28, 065003 (2013).

[7] M. Aziziha, R. Beesley, J. R. Magers, N. Mottaghi, M. B. Holcomb, J. P. Lewis, M. S. Seehra, and M. B. Johnson, Electronic State and Concentration of Fe3+ in CuAll-Fe O2 Determined by Magnetic Measurements, Journal of Magnetism and Magnetic Materials 471, 495 (2019).

[8] M. Aziziha, S. A. Byard, R. Beesely, J. P. Lewis, M. S. Seehra, and M. B. Johnson, Magnetic Properties of Fe-Doped CuAlO 2 and Role of Impurities, AIP Advances 9, 035030 (2019).

[9] W. Setyawan and S. Curtarolo, High-Throughput Electronic Band Structure Calculations: Challenges and Tools, Computational Materials Science 49, 299 (2010).

[10] S. Gilliland, J. Pellicer-Porres, A. Segura, A. Muñoz, P. Rodríguez-Hernández, D. Kim, M. S. Lee, and T. Y. Kim, Electronic Structure of CuAlO2 and $\mathrm{CuScO} 2$ Delafossites under Pressure, Physica Status Solidi (b) 244, 309 (2007).

[11] R.-S. Yu, S.-C. Liang, C.-J. Lu, D.-C. Tasi, and F.-S. Shieu, Characterization and Optoelectronic Properties of P-Type N-Doped CuAlO2 Films, Applied Physics Letters 90, 191117 (2007).

[12] A. N. Banerjee and K. K. Chattopadhyay, Recent Developments in the Emerging Field of Crystalline P-Type Transparent Conducting Oxide Thin Films, Progress in Crystal Growth and Characterization of Materials 50, 52 (2005).

[13] J. Robertson, P. W. Peacock, M. D. Towler, and R. Needs, Electronic Structure of P-Type Conducting Transparent Oxides, Thin Solid Films 411, 96 (2002).

[14] R. Gillen and J. Robertson, Band Structure Calculations of CuAlO2, CuGaO2, CuInO2 and CuCrO2 by Screened Exchange, Phys. Rev. B 84, 035125 (2011).

[15] J. Pellicer-Porres, D. Martínez-García, A. Segura, P. Rodríguez-Hernández, A. Muñoz, J. C. Chervin, N. Garro, and D. Kim, Pressure and Temperature Dependence of the Lattice Dynamics of $\mathrm{Cu} A \mathrm{Al} O 2$ Investigated by Raman Scattering Experiments and Ab Initio Calculations, Physical Review B 74, (2006).

[16] D. O. Scanlon and G. W. Watson, Conductivity Limits in CuAlO 2 from Screened-Hybrid Density Functional Theory, The Journal of Physical Chemistry Letters 1, 3195 (2010).

[17] J. Tate, H. L. Ju, J. C. Moon, A. Zakutayev, A. P. Richard, J. Russell, and D. H. McIntyre, Origin of p-Type Conduction in Single-Crystal CuAlO 2, Physical Review B 80, (2009). 
[18] C. Mondelli, A. P. Amrute, T. Schmidt, and J. Pérez-Ramírez, A Delafossite-Based Copper Catalyst for Sustainable Cl2 Production by $\mathrm{HCl}$ Oxidation, Chemical Communications 47, 7173 (2011).

[19] S. Y. Choi, C.-D. Kim, D. S. Han, and H. Park, Facilitating Hole Transfer on Electrochemically Synthesized P-Type CuAlO ${ }_{2}$ Films for Efficient Solar Hydrogen Production from Water, Journal of Materials Chemistry A 5, 10165 (2017).

[20] N. Koriche, A. Bouguelia, A. Aider, and M. Trari, Photocatalytic Hydrogen Evolution over Delafossite, International Journal of Hydrogen Energy 30, 693 (2005).

[21] N. Koriche, A. Bouguelia, and M. Trari, Photocatalytic Hydrogen Production over New Oxide CuLaO2.62CuLaO2.62, International Journal of Hydrogen Energy 31, 1196 (2006).

[22] Q.-M. Zhao, Z.-Y. Zhao, Q.-L. Liu, G.-Y. Yao, and X.-D. Dong, Delafossite $\mathrm{CuGaO}_{2}$ as Promising Visible-Light-Driven Photocatalyst: Synthesize, Properties, and Performances, Journal of Physics D: Applied Physics 53, 135102 (2020).

[23] S. Saadi, A. Bouguelia, A. Derbal, and M. Trari, Hydrogen Photoproduction over New Catalyst CuLaO2, Journal of Photochemistry and Photobiology A: Chemistry 187, 97 (2007).

[24] S. Santra, N. S. Das, and K. K. Chattopadhyay, Wide Band Gap P-Type Nanocrystalline CuBO2 as a Novel UV Photocatalyst, Materials Research Bulletin 48, 2669 (2013).

[25] S. Kato, N. Takagi, K. Saito, and M. Ogasawara, Synthesis of Delafossite-Type Ag $0.9 \mathrm{MnO}$ 2 by the Precipitation Method at Room Temperature, ACS Omega 4, 9763 (2019).

[26] Y. Maruyama, H. Irie, and K. Hashimoto, Visible Light Sensitive Photocatalyst, Delafossite

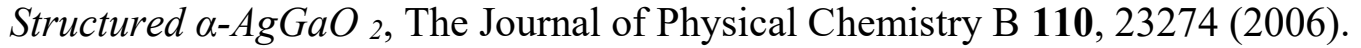

[27] T. Zhao, Q.-L. Liu, and Z.-Y. Zhao, High-Throughput Screening Delafossite CuMO 2 (M= IIIA, 3d, 4d, 5d, and RE) Optoelectronic Functional Materials Based on First-Principles Calculations, The Journal of Physical Chemistry C 123, 14292 (2019).

[28] K. Ramesha, A. S. Prakash, M. Sathiya, G. Madras, and A. K. Shukla, Synthesis and Photocatalytic Properties of Ag[Li1/3Ru2/3]O2: A New Delafossite Oxide, Materials Science and Engineering: B 176, 141 (2011).

[29] W. C. Sheets, E. S. Stampler, M. I. Bertoni, M. Sasaki, T. J. Marks, T. O. Mason, and K. R. Poeppelmeier, Silver Delafossite Oxides, Inorganic Chemistry 47, 2696 (2008).

[30] S. Ouyang, N. Kikugawa, D. Chen, Z. Zou, and J. Ye, A Systematical Study on Photocatalytic Properties of $\mathrm{AgMO}_{2}(M=\mathrm{Al}$, Ga, In): Effects of Chemical Compositions, Crystal Structures, and Electronic Structures, The Journal of Physical Chemistry C 113, 1560 (2009).

[31] J. E. Park, Y. Hu, J. W. Krizan, Q. D. Gibson, U. T. Tayvah, A. Selloni, R. J. Cava, and A. B. Bocarsly, Stable Hydrogen Evolution from an $\mathrm{AgRhO}{ }_{2}$ Photocathode under Visible Light, Chemistry of Materials 30, 2574 (2018).

[32] K. Rajeshwar, M. K. Hossain, R. T. Macaluso, C. Janáky, A. Varga, and P. J. Kulesza, Review-Copper Oxide-Based Ternary and Quaternary Oxides: Where Solid-State Chemistry Meets Photoelectrochemistry, Journal of The Electrochemical Society 165, H3192 (2018).

[33] A. Derbal, S. Omeiri, A. Bouguelia, and M. Trari, Characterization of New Heterosystem $\mathrm{CuFeO} / \mathrm{SnO} 2$ Application to Visible-Light Induced Hydrogen Evolution, International Journal of Hydrogen Energy 33, 4274 (2008). 
[34] J. Vidal, F. Trani, F. Bruneval, M. A. L. Marques, and S. Botti, Effects of Electronic and Lattice Polarization on the Band Structure of Delafossite Transparent Conductive Oxides, Physical Review Letters 104, (2010).

[35] D. CRIBIER, Lattice Dynamics (1965).

[36] X. Gonze and C. Lee, Dynamical Matrices, Born Effective Charges, Dielectric Permittivity Tensors, and Interatomic Force Constants from Density-Functional Perturbation Theory, Physical Review B 55, 10355 (1997).

[37] P. Giannozzi, S. de Gironcoli, P. Pavone, and S. Baroni, Ab Initio Calculation of Phonon Dispersions in Semiconductors, Physical Review B 43, 7231 (1991).

[38] G. Kresse, Ab Initio Molecular Dynamics for Liquid Metals, 8 (1995).

[39] K. Parlinski, Z. Q. Li, and Y. Kawazoe, First-Principles Determination of the Soft Mode in Cubic $\mathrm{ZrO}$ 2, Physical Review Letters 78, 4063 (1997).

[40] L. Bellaiche and D. Vanderbilt, Virtual Crystal Approximation Revisited: Application to Dielectric and Piezoelectric Properties of Perovskites, Physical Review B 61, 7877 (2000).

[41] P. Soven, Coherent-Potential Model of Substitutional Disordered Alloys, Physical Review 156, 809 (1967).

[42] S. Ghosh, P. L. Leath, and M. H. Cohen, Phonons in Random Alloys: The Itinerant Coherent-Potential Approximation, Physical Review B 66, (2002).

[43] F. Körmann, Y. Ikeda, B. Grabowski, and M. H. F. Sluiter, Phonon Broadening in High Entropy Alloys, Npj Computational Materials 3, (2017).

[44] P. G. Klemens, The Scattering of Low-Frequency Lattice Waves by Static Imperfections, Proceedings of the Physical Society. Section A 68, 1113 (1955).

[45] H. R. Seyf and A. Henry, A Method for Distinguishing between Propagons, Diffusions, and Locons, Journal of Applied Physics 120, 025101 (2016).

[46] H. R. Seyf, L. Yates, T. L. Bougher, S. Graham, B. A. Cola, T. Detchprohm, M.-H. Ji, J. Kim, R. Dupuis, W. Lv, and A. Henry, Rethinking Phonons: The Issue of Disorder, Npj Computational Materials 3, (2017).

[47] R. P. Feynman, Forces in Molecules, Physical Review 56, 340 (1939).

[48] M. Aziziha, S. Akbarshahi, S. Ghosh, P. Pramanik, J. P. Lewis, A. H. Romero, S. Thota, M. S. Seehra, and M. B. Johnson, Phonon Dynamics in Anisotropic Dilute $\mathrm{CuAl}{ }_{1-\times} \mathrm{Fe}_{\times} \mathrm{O}_{2}$ Delafossite Alloys by a Weighted Dynamical Matrix Approach, The Journal of Physical Chemistry C 123, 30604 (2019).

[49] M. Aziziha and S. Akbarshahi, Phonon Dispersion Calculation for Binary Alloys Using WDM Approach, ArXiv:2008.00661 [Cond-Mat] (2020).

[50] M. Aziziha and S. Akbarshahi, Impact of Scattering on Phonon Behavior of Alloys, ArXiv:2008.02652 [Cond-Mat] (2020).

[51] M. Aziziha and S. Akbarshahi, Vibrational Entropy Investigation in High Entropy Alloys, ArXiv:2008.03338 [Cond-Mat] (2020).

[52] S. Vojkovic, J. Fernandez, S. Elgueta, F. E. Vega, S. D. Rojas, R. A. Wheatley, B. Seifert, S. Wallentowitz, and A. L. Cabrera, Band Gap Determination in Multi-Band-Gap CuFeO2 Delafossite Epitaxial Thin Film by Photoconductivity, SN Appl. Sci. 1, 1322 (2019).

[53] H. Kizaki, K. Sato, and H. Katayama-Yoshida, Materials Design of CuAlO ${ }_{2}$-Based Dilute Magnetic Semiconductors by First-Principles Calculations and Monte Carlo Simulations, Japanese Journal of Applied Physics 47, 6488 (2008). 
[54] M. Aziziha, S. Akbarshahi, S. Ghosh, P. Pramanik, R. Sooriyagoda, A. H. Romero, S. Thota, A. D. Bristow, M. S. Seehra, and M. B. Johnson, Optical Properties of Dilute CuAl1-XFexO2 Delafossite Alloys, Frontiers in Optics / Laser Science JM6A. 31 (2020).

[55] A. P. Amrute, Z. Łodziana, C. Mondelli, F. Krumeich, and J. Pérez-Ramírez, Solid-State Chemistry of Cuprous Delafossites: Synthesis and Stability Aspects, Chemistry of Materials 25, 4423 (2013).

[56] J. W. Lekse, M. K. Underwood, J. P. Lewis, and C. Matranga, Synthesis, Characterization, Electronic Structure, and Photocatalytic Behavior of $\mathrm{CuGaO}{ }_{2}$ and $\mathrm{CuGa}{ }_{1-\mathrm{x}} \mathrm{Fe}_{\mathrm{x}} \mathrm{O}_{2}$ ( $\mathrm{x}$ $=0.05,0.10,0.15,0.20)$ Delafossites, The Journal of Physical Chemistry C 116, 1865 (2012).

[57] P. Scherrer, Bestimmung Der Inneren Struktur Und Der Größe von Kolloidteilchen Mittels Röntgenstrahlen, in Kolloidchemie Ein Lehrbuch, edited by R. Zsigmondy (Springer Berlin Heidelberg, Berlin, Heidelberg, 1912), pp. 387-409.

[58] K. Khanmohammadi Chenab, B. Sohrabi, M. R. Zamani Meymian, and S. V. Mousavi, Naphthoquinone Derivative-Based Dye for Dye-Sensitized Solar Cells: Experimental and Computational Aspects, Materials Research Express 6, 085537 (2019).

[59] G. K. Williamson and W. H. Hall, X-Ray Line Broadening from Filed Aluminium and Wolfram, Acta Metallurgica 1, 22 (1953).

[60] D. L. and H. Bish S. A., Quantitative Phase Analysis Using the Rietveld Method, J. Appl. Cryst. 21, 86 (1988).

[61] A. G. De La Torre, S. Bruque, and M. A. G. Aranda, Rietveld Quantitative Amorphous Content Analysis, Journal of Applied Crystallography 34, 196 (2001).

[62] L. Vegard, Die Konstitution Der Mischkristalle Und Die Raumfüllung Der Atome, Zeitschrift Für Physik 5, 17 (1921).

[63] Vegard L., XV. Die Röntgenstrahlen Im Dienste Der Erforschung Der Materie, Zkri 67, 239 (2015).

[64] L. V. D. phil, LV. Results of Crystal Analysis.-III., The London, Edinburgh, and Dublin Philosophical Magazine and Journal of Science 32, 505 (1916).

[65] L. V. D. phil, VI. Results of Crystal Analysis, The London, Edinburgh, and Dublin Philosophical Magazine and Journal of Science 32, 65 (1916).

[66] J. I. Goldstein, D. E. Newbury, P. Echlin, D. C. Joy, C. E. Lyman, E. Lifshin, L. Sawyer, and J. R. Michael, Scanning Electron Microscopy and X-Ray Microanalysis, Third (Kluwer Academic/Plenum publishers, 2003).

[67] M. Moradi-Haji Jafan, M.-R. Zamani-Meymian, R. Rahimi, and M. Rabbani, The Effect of Solvents and the Thickness on Structural, Optical and Electrical Properties of ITO Thin Films Prepared by a Sol-Gel Spin-Coating Process, Journal of Nanostructure in Chemistry 4, (2014).

[68] J. Matthew, Surface Analysis by Auger and X-ray Photoelectron Spectroscopy (IMPublications, Chichester, UK and SurfaceSpectra, Manchester, UK, 2003).

[69] D. J. Miller, M. C. Biesinger, and N. S. McIntyre, Interactions of CO2 and CO at Fractional Atmosphere Pressures with Iron and Iron Oxide Surfaces: One Possible Mechanism for Surface Contamination?, Surf. Interface Anal. 33, 299 (2002).

[70] M. Born and R. Oppenheimer, Zur Quantentheorie der Molekeln, Ann. Phys. 389, 457 (1927).

[71] P. Hohenberg and W. Kohn, Inhomogeneous Electron Gas, Physical Review 136, B864 (1964). 
[72] W. Kohn and L. J. Sham, Self-Consistent Equations Including Exchange and Correlation Effects, Physical Review 140, A1133 (1965).

[73] D. M. Ceperley and B. J. Alder, Ground State of the Electron Gas by a Stochastic Method, Phys. Rev. Lett. 45, 566 (1980).

[74] J. P. Perdew, K. Burke, and Y. Wang, Generalized Gradient Approximation for the Exchange-Correlation Hole of a Many-Electron System, Phys. Rev. B 54, 16533 (1996).

[75] J. P. Perdew, K. Burke, and M. Ernzerhof, Generalized Gradient Approximation Made Simple, Physical Review Letters 77, 3865 (1996).

[76] V. I. Anisimov, J. Zaanen, and O. K. Andersen, Band Theory and Mott Insulators: Hubbard U Instead of Stoner I, Physical Review B 44, 943 (1991).

[77] G. Kresse and J. Furthmüller, Efficiency of Ab-Initio Total Energy Calculations for Metals and Semiconductors Using a Plane-Wave Basis Set, Computational Materials Science 6, 15 (1996).

[78] G. Kresse and D. Joubert, From Ultrasoft Pseudopotentials to the Projector AugmentedWave Method, Physical Review B 59, 1758 (1999).

[79] G. Kresse, J. Furthmüller, and J. Hafner, Ab Initio Force Constant Approach to Phonon Dispersion Relations of Diamond and Graphite, Europhysics Letters (EPL) 32, 729 (1995).

[80] P. E. Blöchl, Projector Augmented-Wave Method, Physical Review B 50, 17953 (1994).

[81] R. Grau-Crespo, S. Hamad, C. R. A. Catlow, and N. H. de Leeuw, Symmetry-Adapted Configurational Modelling of Fractional Site Occupancy in Solids, Journal of Physics: Condensed Matter 19, 256201 (2007).

[82] G. I. Csonka, J. P. Perdew, A. Ruzsinszky, P. H. T. Philipsen, S. Lebègue, J. Paier, O. A. Vydrov, and J. G. Ángyán, Assessing the Performance of Recent Density Functionals for Bulk Solids, Physical Review B 79, (2009).

[83] M. Aziziha, M. Motamedifar, and S. Mahdavifar, Multicritical Point in the OneDimensional Quantum Compass Model, Acta Physica Polonica B 44, 221 (2013).

[84] M. C. Gao, High-Entropy Alloys (Springer Science+Business Media, New York, NY, 2016).

[85] F. Tian, A Review of Solid-Solution Models of High-Entropy Alloys Based on Ab Initio Calculations, Frontiers in Materials 4, (2017).

[86] Y. Wang, C. L. Zacherl, S. Shang, L.-Q. Chen, and Z.-K. Liu, Phonon Dispersions in Random Alloys: A Method Based on Special Quasi-Random Structure Force Constants, Journal of Physics: Condensed Matter 23, 485403 (2011).

[87] S. de Gironcoli, Phonons in Si-Ge Systems: An Ab Initio Interatomic-Force-Constant Approach, Physical Review B 46, 2412 (1992).

[88] Z. Cheng, J. Zhai, Q. Zhang, and Y. Ke, Auxiliary Coherent Medium Theory for Lattice Vibrations in Random Binary Alloys with Mass and Force-Constant Disorders, Physical Review B 99, (2019).

[89] S. V. Koniakhin, O. I. Utesov, I. N. Terterov, A. V. Siklitskaya, A. G. Yashenkin, and D. Solnyshkov, Raman Spectra of Crystalline Nanoparticles: Replacement for the Phonon Confinement Model, The Journal of Physical Chemistry C 122, 19219 (2018).

[90] A. S. Barker, J. L. Merz, and A. C. Gossard, Study of Zone-Folding Effects on Phonons in Alternating Monolayers of GaAs-AlAs, Physical Review B 17, 3181 (1978).

[91] A. H. Romero, M. Cardona, R. K. Kremer, R. Lauck, G. Siegle, J. Serrano, and X. C. Gonze, Lattice Properties of $\mathrm{Pb} X(X=S, S e, T e)$ : Experimental Studies and Ab Initio Calculations Including Spin-Orbit Effects, Physical Review B 78, (2008). 
[92] P. G. Etchegoin, M. Cardona, R. Lauck, R. J. H. Clark, J. Serrano, and A. H. Romero, Temperature-Dependent Raman Scattering of Natural and Isotopically Substituted PbS, Physica Status Solidi (b) 245, 1125 (2008).

[93] A. Togo and I. Tanaka, First Principles Phonon Calculations in Materials Science, Scripta Materialia 108, 1 (2015).

[94] K. Ueda, T. Hase, H. Yanagi, H. Kawazoe, H. Hosono, H. Ohta, M. Orita, and M. Hirano, Epitaxial Growth of Transparent P-Type Conducting CuGaO2 Thin Films on Sapphire (001) Substrates by Pulsed Laser Deposition, J. Appl. Phys. 89, 5 (2001).

[95] B. Falabretti and J. Robertson, Electronic Structures and Doping of SnO2, CuAlO2, and CuInO2, Journal of Applied Physics 102, 123703 (2007).

[96] A. J. Cohen, P. Mori-Sanchez, and W. Yang, Insights into Current Limitations of Density Functional Theory, Science 321, 792 (2008).

[97] R. Laskowski, N. E. Christensen, P. Blaha, and B. Palanivel, Strong Excitonic Effects in CuAlO 2 Delafossite Transparent Conductive Oxides, Physical Review B 79, (2009).

[98] A. Jain, G. Hautier, S. P. Ong, C. J. Moore, C. C. Fischer, K. A. Persson, and G. Ceder, Formation Enthalpies by Mixing GGA and GGA +U Calculations, Physical Review B 84, (2011).

[99] F. Trani, J. Vidal, S. Botti, and M. A. L. Marques, Band Structures of Delafossite Transparent Conductive Oxides from a Self-Consistent $G W$ Approach, Physical Review B 82, (2010).

[100] E. N. Brothers, A. F. Izmaylov, J. O. Normand, V. Barone, and G. E. Scuseria, Accurate Solid-State Band Gaps via Screened Hybrid Electronic Structure Calculations, The Journal of Chemical Physics 129, 011102 (2008).

[101] G. Kresse and J. Hafner, Ab Initio Molecular Dynamics for Liquid Metals, Phys. Rev. B 47, 558 (1993).

[102] G. Kresse and J. Hafner, Ab Initio Molecular-Dynamics Simulation of the Liquid-MetalAmorphous-Semiconductor Transition in Germanium, Phys. Rev. B 49, 14251 (1994).

[103] S. L. Dudarev, G. A. Botton, S. Y. Savrasov, C. J. Humphreys, and A. P. Sutton, Electron-Energy-Loss Spectra and the Structural Stability of Nickel Oxide: An LSDA+U Study, Phys. Rev. B 57, 1505 (1998).

[104] L.-W. Wang, L. Bellaiche, S.-H. Wei, and A. Zunger, "Majority Representation" of Alloy Electronic States, Phys. Rev. Lett. 80, 4725 (1998).

[105] O. Rubel, A. Bokhanchuk, S. J. Ahmed, and E. Assmann, Unfolding the Band Structure of Disordered Solids: From Bound States to High-Mobility Kane Fermions, Physical Review B 90, (2014).

[106] J. Luo and Y.-J. Lin, Point Defect-Induced Magnetic Properties in CuAlO 2 Films without Magnetic Impurities, Applied Physics A 122, 163 (2016).

[107] K. Park, K. Y. Ko, H.-C. Kwon, and S. Nahm, Improvement in Thermoelectric Properties of $\mathrm{CuAlO}_{2}$ by Adding $\mathrm{Fe}_{2} \mathrm{O}_{3}$, Journal of Alloys and Compounds 437, 1 (2007).

[108] H. Y. Zhang, P. G. Li, C. P. Chen, Q. Y. Tu, and W. H. Tang, Magnetic Properties of

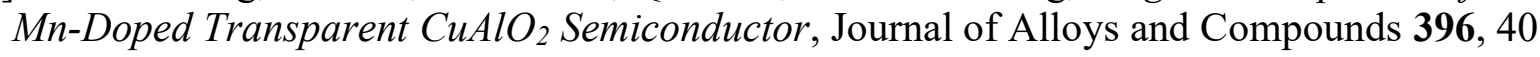
(2005).

[109] C. Chen, C. Dong, B. Wang, J. Huang, and Y. Wang, Synthesis and Room Temperature Ferromagnetism in Fe-Doped CuAlO2 Semiconductor, Journal of Wuhan University of Technology-Mater. Sci. Ed. 28, 500 (2013). 
[110] J. Töpfer, M. Trari, and J.-P. Doumerc, Preparation and Physical Properties of

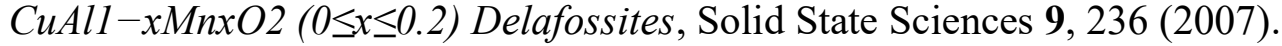

[111] C. Kittel, Introduction to Solid State Physics (Wiley, 1971).

[112] Z. Wang (王正君), L. Pi, M. S. Seehra, J. Bindra, H. van Tol, and N. S. Dalal, Magnetic Studies Reveal Near-Perfect Paramagnetism in the Molecular Semiconductor Vanadyl Phthalocyanine $\left(\mathrm{C}_{32} \mathrm{H}_{16} \mathrm{~N}_{8} \mathrm{VO}\right)$, Journal of Magnetism and Magnetic Materials 422, 386 (2017).

[113] N. Mottaghi, R. B. Trappen, S. Kumari, C.-Y. Huang, S. Yousefi, G. B. Cabrera, M. Aziziha, A. Haertter, M. B. Johnson, M. S. Seehra, and M. B. Holcomb, Observation and Interpretation of Negative Remanent Magnetization and Inverted Hysteresis Loops in a Thin Film of La $0.7 \mathrm{Sr}_{0.3} \mathrm{MnO}_{3}$, Journal of Physics: Condensed Matter 30, 405804 (2018).

[114] Ö. Özdemir, D. J. Dunlop, and T. S. Berquó, Morin Transition in Hematite: Size Dependence and Thermal Hysteresis, Geochemistry, Geophysics, Geosystems 9, (2008).

[115] Ö. Özdemir and D. J. Dunlop, Hysteresis and Coercivity of Hematite, Journal of Geophysical Research: Solid Earth 119, 2582 (2014).

[116] M. T. Dove, Introduction to the Theory of Lattice Dynamics, JDN 12, 123 (2011).

[117] Altmann and P. Herzig, Point-Group Theory Tables (Oxford Science Publications, 1994).

[118] G. F. Koster, J. O. Dimmock, R. G. Wheeler, and H. Statz, Properties of the Thirty-Two Point Groups (M.I.T. press, Cambridge, Massachusetts, 1963).

[119] D. J. Gardiner, Practical Raman Spectroscopy (Springer-Verlag, 1989).

[120] D. A. Skoog, F. J. Holler, and S. R. Crouch, Principles of Instrumental Analysis, Sixth (David Harris, n.d.).

[121] M. Fox, OPTICAL PROPERTIES OF SOLIDS (OUP Oxford, 2010).

[122] O. A. Yassin, S. N. Alamri, and A. A. Joraid, Effect of Particle Size and Laser Power on the Raman Spectra of CuAlO ${ }_{2}$ Delafossite Nanoparticles, Journal of Physics D: Applied Physics 46, 235301 (2013).

[123] R. Liu, Y. Li, B. Yao, Z. Ding, R. Deng, L. Zhang, H. Zhao, and L. Liu, Experimental and First-Principles Study of Photoluminescent and Optical Properties of Na-Doped $\mathrm{CuAlO}_{2}$ : The Role of the $\mathrm{Na} \mathrm{Al}-2 \mathrm{Na}$ i Complex, Journal of Physics D: Applied Physics 48, $335102(2015)$.

[124] W. Lan, J. Q. Pan, C. Q. Zhu, G. Q. Wang, Q. Su, X. Q. Liu, E. Q. Xie, and H. Yan, Role of Oxygen in Structural Properties of Annealed CuAlO2 Films, Journal of Crystal Growth 314, 370 (2011).

[125] A. Garg and R. Rao, Copper Delafossites under High Pressure-A Brief Review of XRD and Raman Spectroscopic Studies, Crystals 8, 255 (2018).

[126] M. S. Seehra, V. Narang, U. K. Geddam, and A. B. Stefaniak, Correlation between XRay Diffraction and Raman Spectra of 16 Commercial Graphene-Based Materials and Their Resulting Classification, Carbon 111, 380 (2017).

[127] N. P. Salke, K. Kamali, T. R. Ravindran, G. Balakrishnan, and R. Rao, Raman Spectroscopic Studies of CuFeO2 at High Pressures, Vibrational Spectroscopy 81, 112 (2015).

[128] Tery L. Barr and Sudipta Seal, Nature of the Use of Adventitious Carbon as a Binding Energy Standard, Journal of Vacuum Science \& Technology A 1239 (1995).

[129] Barbara H. Stuart, Infrared Spectroscopy: Fundamentals and Applications (2004 John Wiley \& Sons, Ltd, 2004).

[130] Boca Raton, Infrared Spectral Interpretation, 1st ed. (CRC Press, 2018). 
[131] Coates, J., Interpretation of Infrared Spectra, A Practical Approach. In Encyclopedia of Analytical Chemistry (2006).

[132] C. Lv, Y. Huang, M. Feng, M. Long, and C. Zhang, Strcture and Infrared Optical Properties Research on Fe-Doped CuAlO2 Semiconductor, in Proceedings of the 5th International Conference on Advanced Design and Manufacturing Engineering (Atlantis Press, Shenzen, China, 2015).

[133] P. Kubelka, New Contributions to the Optics of Intensely Light-Scattering Materials. Part $I, 10$ (n.d.).

[134] P. KUBELKA and F. Munk, Ein Beitrag Zur Optik Der Farbanstriche, Zeit. Für Tekn. Physik 12, 593 (n.d.).

[135] J. Tauc, Optical Properties and Electronic Structure of Amorphous Ge and Si, Materials Research Bulletin 3, 37 (1968).

[136] J. Tauc, R. Grigorovici, and A. Vancu, Optical Properties and Electronic Structure of Amorphous Germanium, Phys. Stat. Sol. (b) 15, 627 (1966).

[137] W. W. Kou and M. S. Seehra, Optical Absorption in Iron Pyrite (Fe S 2 ), Phys. Rev. B 18, 7062 (1978).

[138] K. V. I. Sivaneri, O. Ozmen, M. Aziziha, E. M. Sabolsky, T. H. Evans, D. B. DeVallance, and M. B. Johnson, Robust Polymer-HfO ${ }_{2}$ Thin Film Laminar Composites for Tactile Sensing Applications, Smart Materials and Structures 28, 025002 (2019).

[139] M. R. Zamani Meymian, R. Haji Abdolvahab, and A. Kosari Mehr, Fractal Characteristics of TiO2-Ag Nanocomposite Films Deposited by a Grid-Assisted CoSputtering Method, Applied Surface Science 480, 593 (2019).

[140] M. R. Z. Meymian, A. D. Heravi, and A. K. Mehr, Influence of Bias Voltage on Optical and Structural Characteristics of Cu3N Films Deposited by Reactive RF Magnetron Sputtering in a Pure Nitrogen Atmosphere, Materials Science in Semiconductor Processing 7 (2020).

[141] F. A. Benko and F. P. Koffyberg, Opto-Electronic Properties of p-and n-Type Delafossite, $\mathrm{CuFeO2}$, Journal of Physics and Chemistry of Solids 48, 431 (1987).

[142] Z. Deng, X. Fang, S. Wu, W. Dong, J. Shao, S. Wang, and M. Lei, The Morphologies and Optoelectronic Properties of Delafossite CuFeO2 Thin Films Prepared by PEG Assisted Sol-Gel Method, Journal of Sol-Gel Science and Technology 71, 297 (2014).

[143] K. P. Ong, K. Bai, P. Blaha, and P. Wu, Electronic Structure and Optical Properties of $\mathrm{AFeO}_{2}(\mathrm{~A}=\mathrm{Ag}, \mathrm{Cu})$ within GGA Calculations, Chemistry of Materials 19, 634 (2007).

[144] T. B. Boykin, N. Kharche, G. Klimeck, and M. Korkusinski, Approximate Bandstructures of Semiconductor Alloys from Tight-Binding Supercell Calculations, J. Phys.: Condens. Matter 19, 036203 (2007).

[145] C. Guarneros Aguilar, C. Estrada Moreno, M. Pacio Castillo, and F. Caballero-Briones, Effect of Calcination Temperature on Structure and Thermoelectric Properties of CuAlO2 Powders, J Mater Sci 53, 1646 (2018).

[146] L. Yang and S. J. Miklavcic, Revised Kubelka-Munk Theory III A General Theory of Light Propagation in Scattering and Absorptive Media, J. Opt. Soc. Am. A 22, 1866 (2005).

[147] T. Tangcharoen, J. T-Thienprasert, and C. Kongmark, Effect of Calcination Temperature on Structural and Optical Properties of MAl2O4 $(\mathrm{M}=\mathrm{Ni}, \mathrm{Cu}, \mathrm{Zn})$ Aluminate Spinel Nanoparticles, J Adv Ceram 8, 352 (2019). 
[148] Y. Oh, W. Yang, J. Kim, S. Jeong, and J. Moon, Enhanced Photocurrent of Transparent $\mathrm{CuFeO}{ }_{2}$ Photocathodes by Self-Light-Harvesting Architecture, ACS Appl. Mater. Interfaces 9, 14078 (2017).

[149] D. Xiong, Q. Zhang, S. K. Verma, X.-Q. Bao, H. Li, and X. Zhao, Crystal Structural, Optical Properties and Mott-Schottky Plots of p-Type Ca Doped CuFeO 2 Nanoplates, Materials Research Bulletin 83, 141 (2016).

[150] J. Pellicer-Porres, A. Segura, A. S. Gilliland, A. Muñoz, P. Rodríguez-Hernández, D. Kim, M. S. Lee, and T. Y. Kim, On the Band Gap of CuAlO2 Delafossite, Applied Physics Letters 88, 181904 (2006).

[151] T. R. Senty, B. Haycock, J. Lekse, C. Matranga, H. Wang, G. Panapitiya, A. D. Bristow, and J. P. Lewis, Optical Absorption and Disorder in Delafossites, Applied Physics Letters 111, 012102 (2017).

[152] D. Schrecongost, M. Aziziha, H. Zhang, I. Tung, J. Tessmer, W. Dai, Q. Wang, R. EngelHerbert, H. Wen, Y. N. Picard, and C. Cen, On-Demand Nanoscale Manipulations of Correlated Oxide Phases, Advanced Functional Materials 1905585 (2019). 